\title{
Protein Import into Chloroplasts
}

\author{
Lea Vojta
}

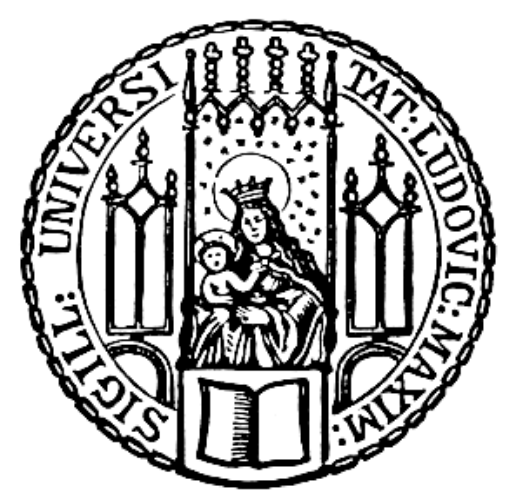

München 2006 



\title{
Protein Import into Chloroplasts
}

\author{
Dissertation \\ Zur Erlangung des Doktorgrades \\ der Fakultät für Biologie \\ der Ludwig-Maximilians-Universität \\ München
}

Vorgelegt von

Lea Vojta

München, 19. Dezember 2006 
Gutachter:

1. Prof. Dr. Jürgen Soll

2. Prof. Dr. Dario Leister

Tag der mündlichen Prüfung: 19. Dezember 2006 


\section{Contents}

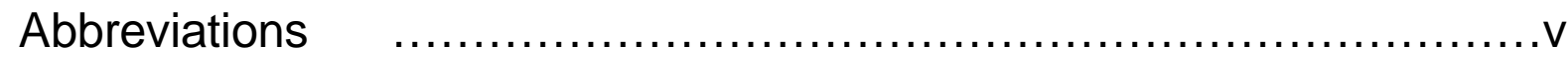

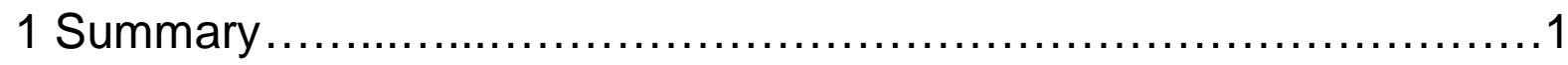

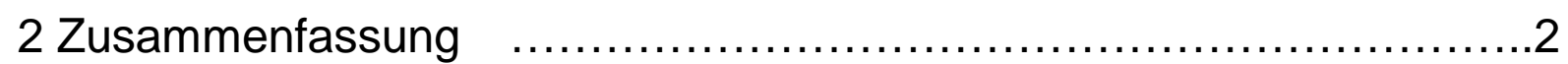

3 Introduction $\quad$.

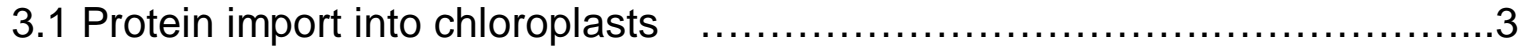

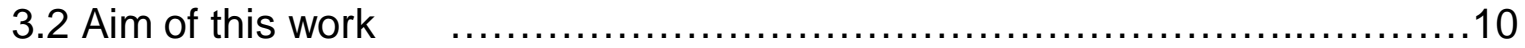

3.2.1 Inner envelope membrane protein Tic110 ............................10

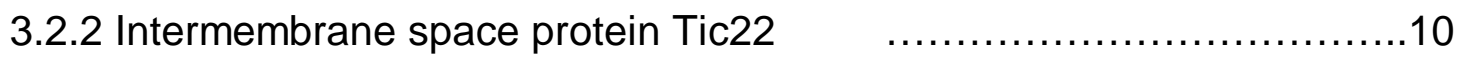

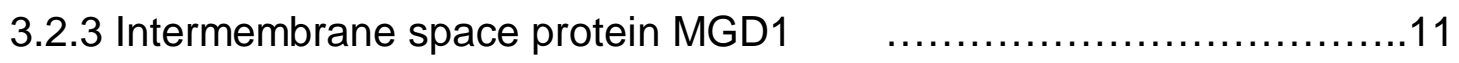

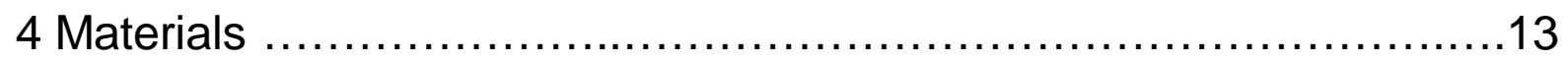

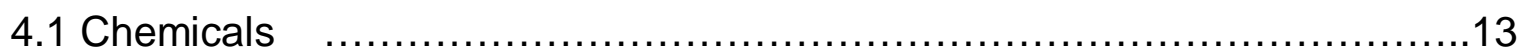

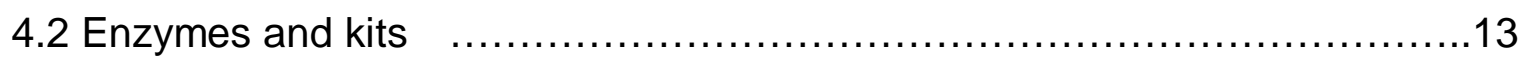

4.3 Molecular weight and size markers $\quad$.........................................

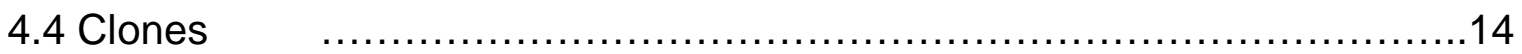

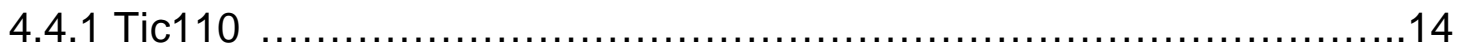

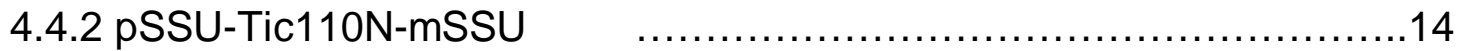

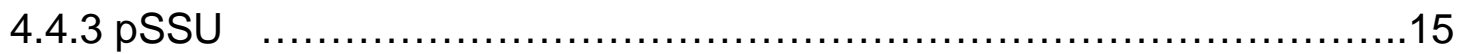

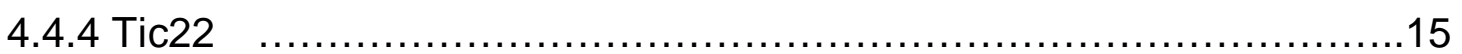

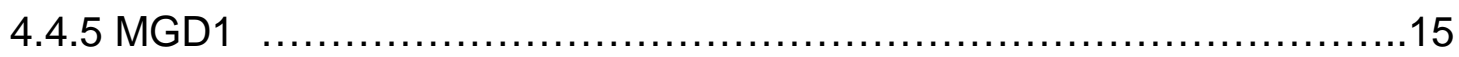

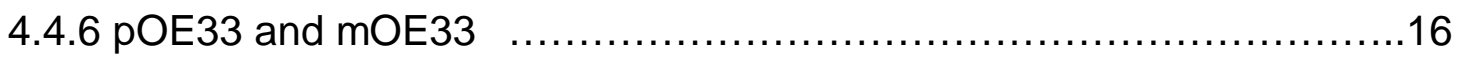

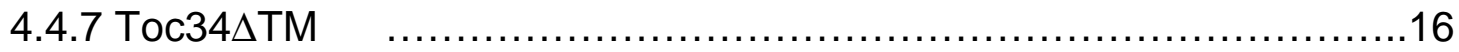

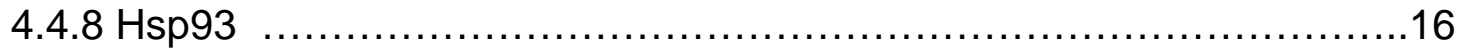

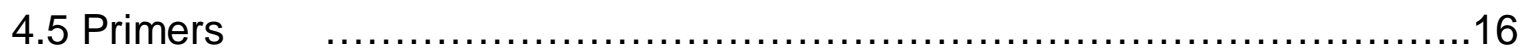

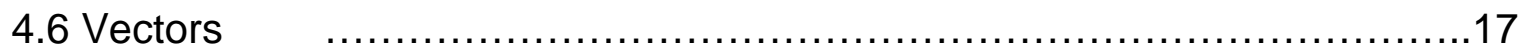

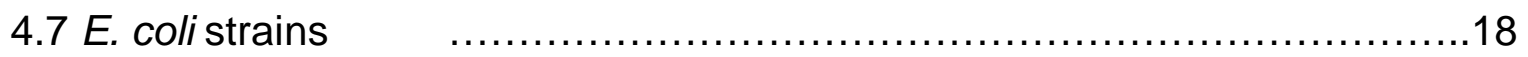

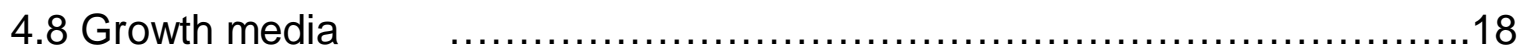

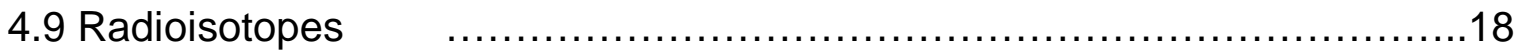

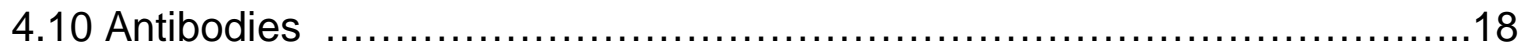

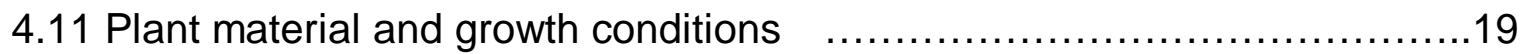

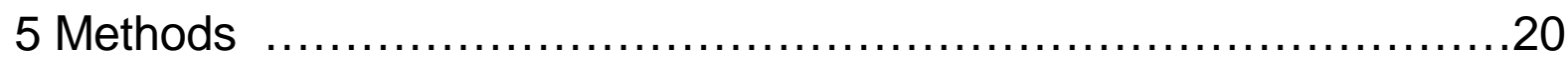

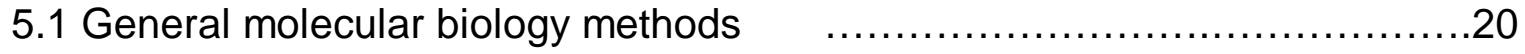




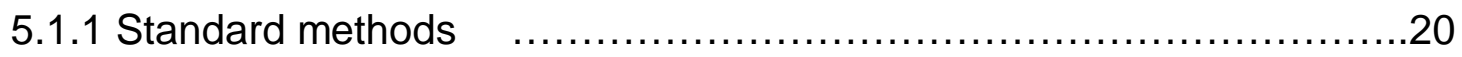

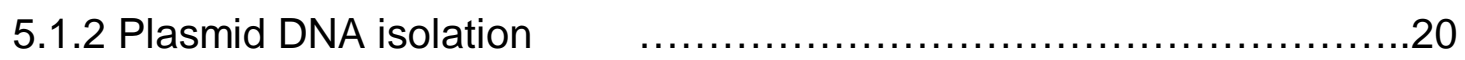

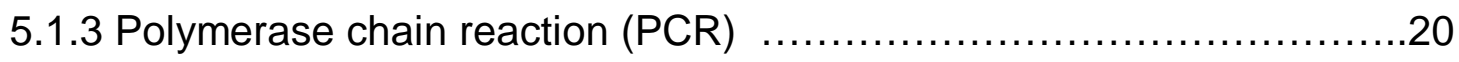

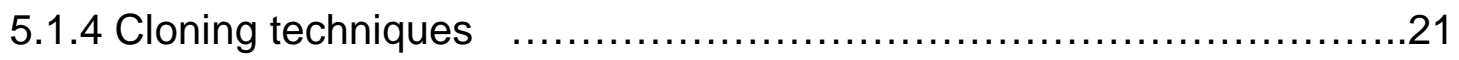

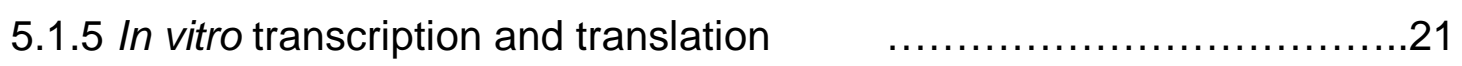

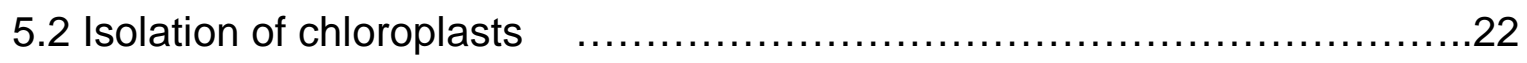

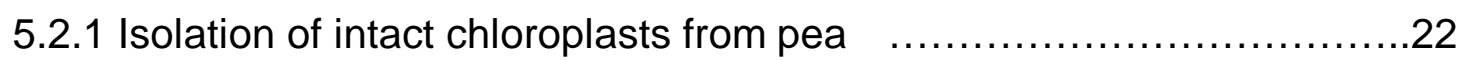

5.2.2 Isolation of intact chloroplasts from spinach $\quad$.......................22

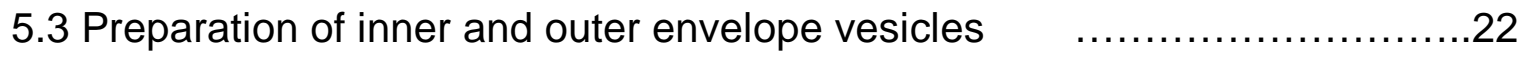

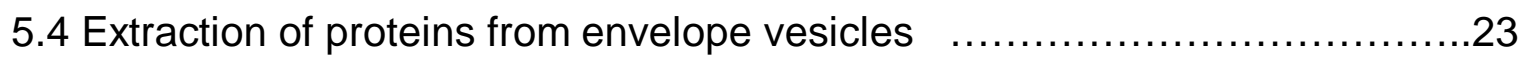

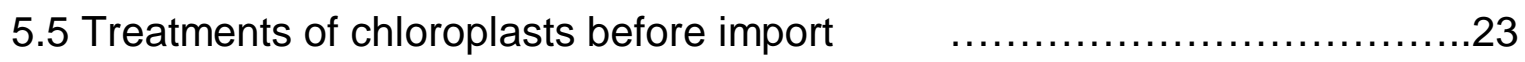

5.5.1 ATP depletion from chloroplasts and in vitro translation product $\quad . .23$

5.5.2 Protease pretreatment of isolated intact chloroplasts $\ldots \ldots \ldots \ldots \ldots \ldots . . . .24$

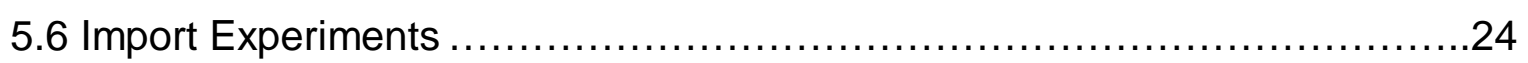

5.6.1 Import of radioactively labelled proteins into intact chloroplasts $\quad .24$

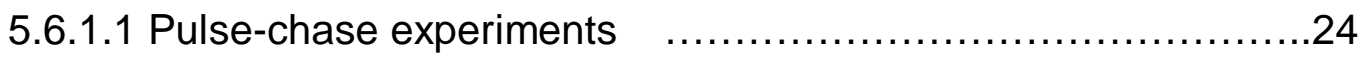

5.6.1.2 Competition with pOE33 and mOE33 proteins $\ldots \ldots \ldots \ldots \ldots \ldots . . .25$

5.6.1.3 Competition for import by the cytosolic domain

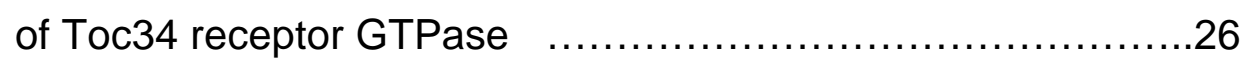

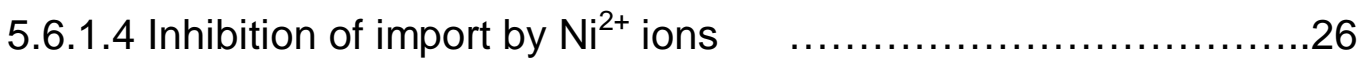

5.6.1.5 Protease posttreatment of intact chloroplasts $\quad$..................26

5.6.2 Suborganellar localization of imported constructs $\quad$.................26

5.6.2.1 Chloroplast fractionation into soluble and insoluble fractions ..26

5.6.2.2 Extraction of proteins with 6M Urea

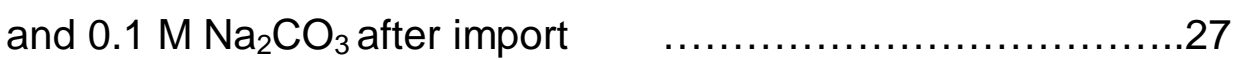

5.6.2.3 Chemical crosslinking and immunoprecipitation ..................27

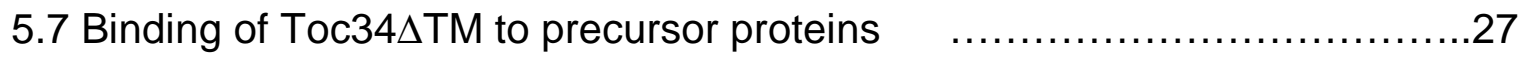

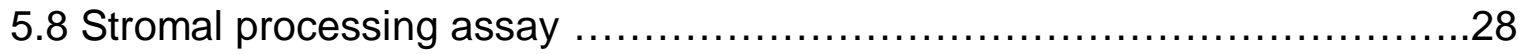

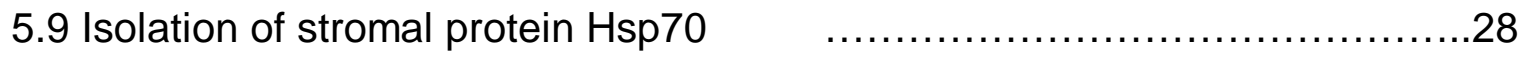

5.10 Protein overexpression and purification $\quad \ldots \ldots \ldots \ldots \ldots \ldots \ldots \ldots \ldots \ldots \ldots \ldots \ldots \ldots \ldots . .29$

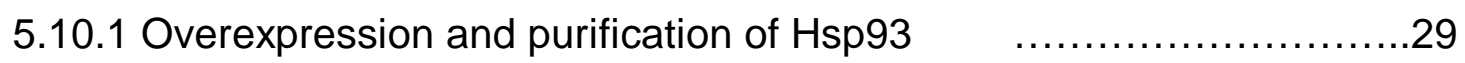

5.10.2 Overexpression and purification of MGD1-P $\ldots \ldots \ldots \ldots \ldots \ldots \ldots \ldots . .29$

5.10.3 Overexpression and purification of pOE33 and mOE33 $\quad \ldots \ldots \ldots . . .30$

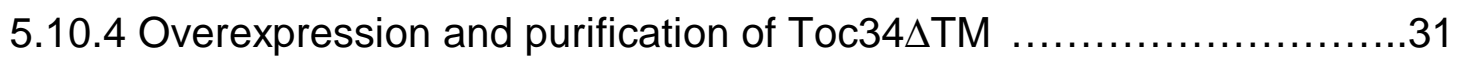




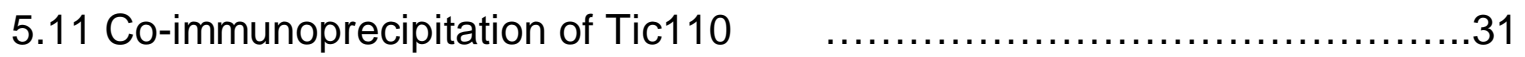

5.12 Methods for separation and identification of proteins ..........................

5.12.1 SDS-Polyacrylamide-Gel-Electrophoresis (SDS-PAGE) ..........32

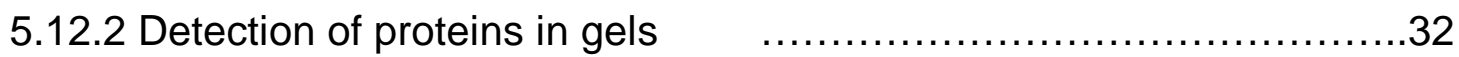

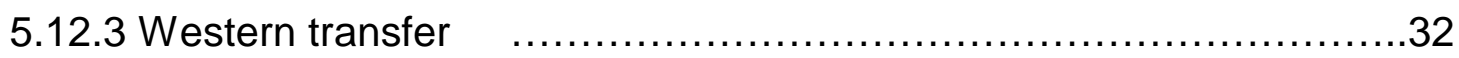

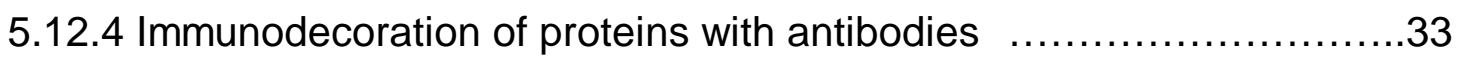

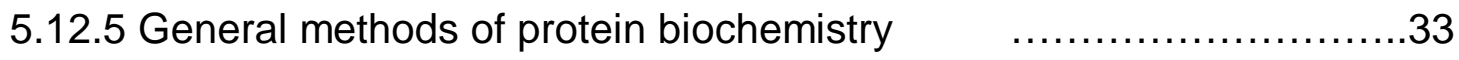

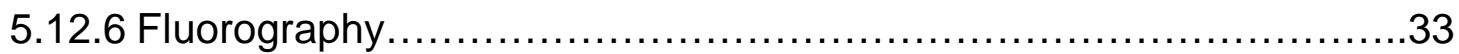

6 Results $\quad$.

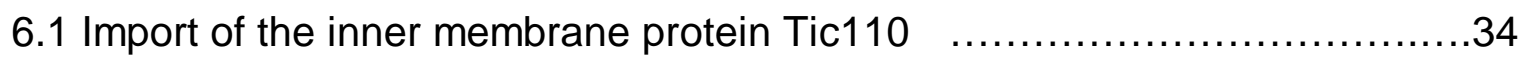

6.1.1 Import of Tic110 and pSSU-Tic110N-mSSU $\quad \ldots \ldots \ldots \ldots \ldots \ldots \ldots . \ldots \ldots$

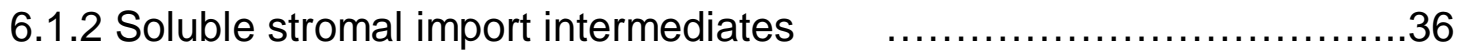

6.1.3 Purification of the stromal chaperone Hsp70 ........................39

6.1.4 Overexpression and purification of the stromal chaperone Hsp93 $\quad . .40$

6.1.5 Immunoprecipitation of Tic110 by stromal chaperones .................42

6.2 Import properties of the chloroplastic intermembrane

space proteins Tic22 and MGD1 ........................................ 44

6.2.1 Energy dependence of Tic22 and MGD1 import ......................48

6.2.2 Processing of the precursor proteins pTic22 and pMGD1 _.........54

6.2.3 pTic22 and pMGD1 depend on proteinaceous components

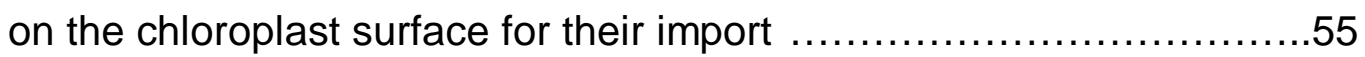

6.2.4 pTic22 and pMGD1 compete with pOE33

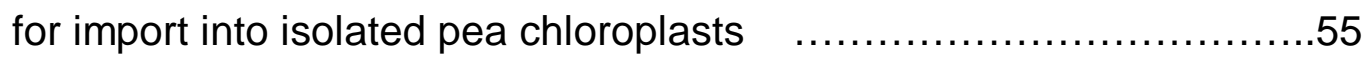

6.2.5 Toc34 $4 \mathrm{TM}$ competes with the endogenous Toc34 for binding

to pTic22 and pMGD1 prior to import into isolated chloroplasts..........58

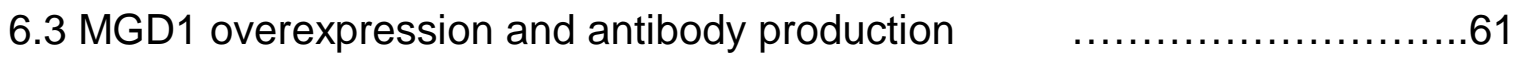

6.4 Chemical crosslinking and immunoprecipitation $\quad$..........................63

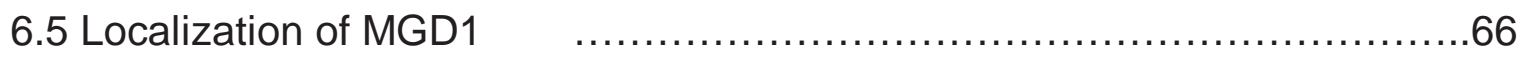

7 Discussion $\quad$.......................................................

7.1 Import pathway of Tic110 into the inner

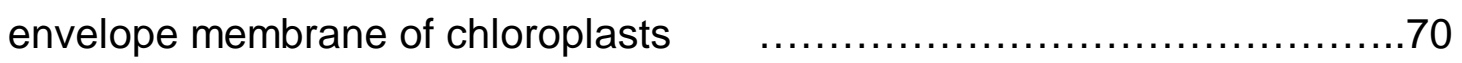

7.2 Import pathway of intermembrane space protein Tic22 $\quad$...................72

7.3 Import and localization of MGD1 in the

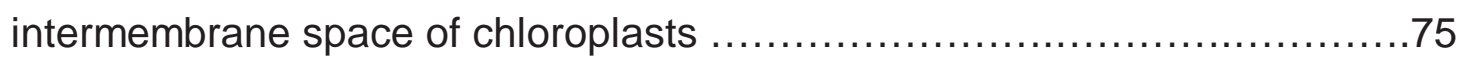




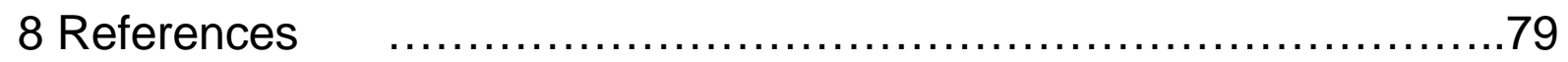

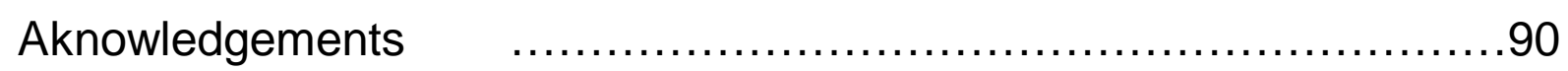

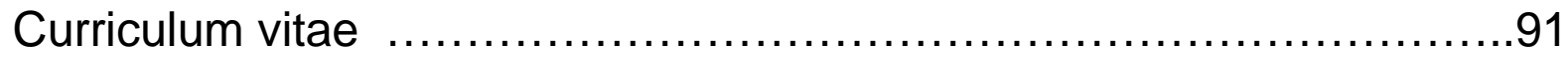

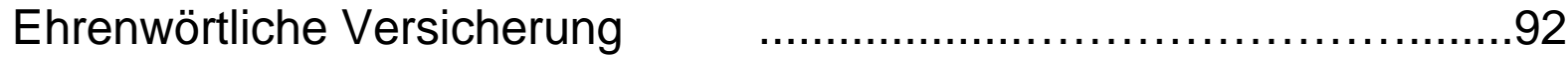




\section{Abbreviations}

Aa

AMP-PNP

ATP

ATP- $\gamma-\mathrm{S}$

$\beta-\mathrm{MeEtOH}$

BSA

BCIP

bp

cap

C-terminus

DeMa

DGDG

DMSO

DSP

DTT

EDTA

GMP-PNP

GTP

GTPase

GTP- $\gamma-S$

HEPES

Hsp

IEP

IPTG

KPi

$\mathrm{m}$

MGDG

MGDG Synthase

MOPS

$\mathrm{N}$-terminus
Amino acid

Adenosine-5' $-[\beta, \gamma$ - imido $]$ triphosphate = Adenylyl-imidodiphosphate

Adenosine-5' -triphosphate

Adenosine-5'-O (3-thiophosphate)

$\beta$-mercapto ethanol (2- mercapto ethanol)

Bovine serum albumin

5-bromo-4-chloro-3-indolyphosphate-p-toluid salt

Base pair

$\mathrm{m}^{7}$-Guanosin (5') ppp (5')Guanosin

Carboxy terminus

Decylmaltoside

Digalactosyldiacylglycerol

Dimethyl sulfoxyde

Dithiobis-succinimidyl-proprionate

Dithiothreitol

Ethylenediaminetetraacetic acid

Guanosine 5'-[ $\beta, \gamma$-imido]triphosphate

Guanosine-5'-triphosphate

Guanosine triphosphate hydrolase

Guanosine 5' -[ $\gamma$-thio]triphosphate

N-2-hydroxyethylpiperasin-N'-2-ethasulfonate

Heat shock protein

Inner envelope protein

Isopropylthiogalactoside

Potassium phosphate buffer

Mature form of; mature protein

Monogalactosyldiacylglycerol

Monogalactosyldiacylglicerol synthase

= UDPgalactose:1,2-diacylglycerol 3- $\beta$-D-galactosyltransferase

Morpholinopropansulfonate

Amino terminus 


$\begin{array}{ll}\text { NAD } & \text { Nicotinamide adenine dinucleotide } \\ \text { NaPi } & \text { Sodium phosphate buffer } \\ \text { NBT } & \text { 4-nitrotetrazoliumchlorid-blue-hydrate } \\ \text { NTP } & \text { Ribonucleoside-5'-triphosphate } \\ \text { O.D.600 } & \text { Optical density at } 600 \text { nm } \\ \text { OE33 } & \text { 33-kDa oxygen evolving complex subunit } \\ \text { OEP } & \text { Outer envelope protein } \\ \text { PC } & \text { Phosphatidylcholine } \\ \text { PCR } & \text { Polymerase chain reaction } \\ \text { PEP } & \text { Phosphoenol pyruvat } \\ \text { PG } & \text { Phosphatidylglycerol } \\ \text { PMSF } & \text { Phenylmethane sulfonyl fluoride } \\ \text { PPO } & \text { 2,5-diphenyloxazole } \\ \text { p } & \text { Precursor of } \\ \text { RubisCO } & \text { Ribulose-1,5-bisphosphate carboxlase/oxygenase } \\ \text { SDS } & \text { Sodium dodecyl sulphate } \\ \text { SDS-PAGE } & \text { Sodium dodecyl sulphate polyacrylamide gel electrophoresis } \\ \text { SQDG } & \text { Sulfoqinovosildiacylglycerol } \\ \text { SPP } & \text { Stromal processing peptidase } \\ \text { SSU } & \text { Small subunit of RubisCO } \\ \text { TCA } & \text { Trichloroacetic acid } \\ \text { Tic } & \text { Translocon of the inner envelope membrane of chloroplast } \\ \text { Tris } & \end{array}$




\section{Summary}

Import of a hybrid construct consisting of the transit sequence of SSU, the N-proximal part of mature Tic110 and the mature SSU into chloroplasts led to the appearance of a soluble stromal import intermediate and the proposal that Tic110 might use a re-export pathway from the stroma to the inner envelope membrane. For full length Tic110 no soluble intermediate has been observed yet. One of the goals of this work was to investigate the import pathway of Tic110 in more detail. In this research the soluble stromal intermediate of Tic110 was observed, its re-export to the membrane was followed, and finally, the intermediate was isolated and co-immunoprecipitated with the stromal chaperones Hsp93, Hsp70 and to a lesser extent Cpn60. The obtained results indicate that Tic110, as proposed, uses a re-export pathway (conservative sorting) during its import into the chloroplast inner envelope membrane. Tic110 also requires stromal chaperones for achieving its native conformation, prior to the insertion into the inner envelope membrane. The pathway for targeting to the intermembrane space of chloroplasts had not been intensively studied yet. For this reason, the analysis of two intermembrane space localized proteins was conducted: Tic22, a $22 \mathrm{kDa}$ Ticcomplex protein component, and MGD1, synthase of MGDG, the most abundant galactolipid in nature. Both proteins are nuclear-encoded and synthesized on cytosolic ribosomes with a cleavable N-terminal chloroplast targeting presequence. Tic22 was found to be associated with the outer face of the inner envelope membrane, as well as with the inner face of the outer envelope membrane, even though at a lower level. MGD1 was proposed to be associated with one of the envelopes by weak electrostatic interactions. Import properties of Tic22 and MGD1 and the localization of MGD1 were investigated in this research. Results presented in this thesis show that import of MGD1 is dependent on, and that of Tic22 is enhanced by, but not dependent on, addition of external ATP. Both preproteins need thermolysin sensitive components on the chloroplast surface for successful import. Chemical crosslinking and immunoprecipitation have demonstrated that Tic22 and MGD1 interact with the components of the Toc translocon of the chloroplast outer envelope during their translocation. Import competition experiments showed that both proteins use the Toc machinery of the general import pathway. Therefore, proteins targeted to the intermembrane space seem to use the same translocation mode across the outer envelope as stromal proteins. 


\section{Zusammenfassung}

Der Import in Chloroplasten eines hybriden Konstrukts, das aus dem Transitpeptid von SSU, dem N-proximal Teil von maturem Tic110 und dem maturen SSU besteht, führte zum erscheinen eines löslichen stromalen Importintermediats und zu der Hypothese, dass Tic110 einen Reexport-Weg vom Stroma in die innere Hüllmembran verwenden könnte. Für das Volllängen-Protein Tic110 war kein lösliches Intermediat beobachtet worden. Eines der Ziele dieser Arbeit war den Importpfad von Tic110 ausführlicher untersuchen. In dieser Arbeit wurde das lösliche stromale Intermediat von Tic110 beobachtet, seine Re-Insertion in die Membran wurde verfolgt, und schließlich wurde das Intermediat isoliert und mit den stromalen Chaperonen Hsp93, Hsp70 und in einem kleineren Ausmaß Cpn60 coimmungefällt. Die erhaltenen Ergebnisse zeigen, dass Tic110, einen Reexport-Weg während seines Imports in die innere Hüllmembran von Chloroplasten verwendet. Tic110 benötigt stromale Chaperone, um seine native Konformation vor der Insertion in die innere Hüllmembran zu erreichen. Der Import mechanismus, der die Proteine zum Intermembranraum von Chloroplasten dirigiert, war noch nicht intensiv untersucht worden. Deshalb wurde der Import von zwei im Intermembranraum lokalisierten Proteinen analysiert: Tic22, ein 22 kDa ProteinBestandteil des Tic-Komplexes, und MGD1, Synthase des MGDG, des häufigsten Galactolipid. Beide Proteine werden im Kern kodiert und an den Ribosomen im Cytosol mit einer spaltbaren N-terminalen Präsequenz synthetisiert. Tic22 bindet an die Außenseite der inneren Hüllmembran, und im geringerem Maße an die Innenseite der äußeren Hüllmembran. MGD1 scheint über elektrostatische Interaktionen mit den Hüllmembranen zu interagieren. Das Importverhalten von Tic22 und MGD1 und die Lokalisierung von MGD1 wurden in dieser Arbeit untersucht. Die hier präsentierten Ergebnisse zeigen, dass der Import von MGD1 von extern zugefügtem ATP abhängig ist im Gegensatz zu Tic22, dessen Importeffizienz in Anwesenheit von ATP zunimmt. Beide Vorstufenproteine benötigen Protease-sensitive Komponenten auf der Chloroplast-Oberfläche für den erfolgreichen Import. Chemische Quervernetzungen und Immunfällungen haben gezeigt, dass Tic22 und MGD1 während ihrer Translokation mit den Bestandteilen des Toc Komplexes interagieren. Importkompetitions-Experimente zeigten, dass beide Proteine die Toc Maschinerie des allgemeinen Importwegs verwenden. Daraus folgt, dass diese Proteine des Intermembranraums denselben Weg über die äußere Hüllmembran nutzen wie stromale Proteine. 


\section{Introduction}

Chloroplast originated from an endosymbiotic event, in which an ancestral photosynthetic cyanobacterium was taken up by a heterotrophic host cell that already contained mitochondria (Cavalier-Smith, 2000). Endosymbiotic evolution has resulted in the transfer of genes encoding the vast majority of the protein components of plastids to the nuclear genome (Martin and Herrmann, 1998). In response to this displacement of genetic material, plastids have evolved a system to post-translationally import nuclear encoded preproteins from their site of synthesis on cytoplasmic ribosomes (Keegstra and Cline, 1999, Martin et al., 2002, Jarvis, 2004). The plastid genome encodes 80-200 plastid-localized proteins that are translated on 70S ribosomes and functionally assembled within the plastid (Jarvis and Robinson, 2004). The protein import machinery of chloroplasts has no known functional equivalent in cyanobacteria (Heins et al., 1998, Heins and Soll, 1998). Homologues of import translocon components Toc75, Tic55, Tic22 and Tic20 have been found encoded in the genome of Synechocystis PCC6803 (Heins and Soll, 1998, Reumann and Keegstra, 1999), but Toc159, Toc34 and Tic110 show no clear prokaryotic origin and might have been added to the translocon during the conversion of an endosymbiont to an organelle. Plant cells regulate the import apparatus in concert with the protein demands of the developing plastids (Mullet, 1998).

\subsection{Protein import into chloroplasts}

Most of the nuclear-encoded chloroplast proteins are synthesized with cleavable N-terminal presequences that are necessary and sufficient for targeting to the chloroplast and for translocation across the chloroplast envelope (Keegstra et al., 1989, De Boer and Weisbeek, 1991, Cline and Henry 1996). The targeting sequences are sequentially decoded resulting in the localization of the polypeptide to the appropriate organellar subcompartment: outer and inner envelope membrane, intermembrane space, stroma, thylakoid membranes or thylakoid lumen (Keegstra et al., 1995, Cline and Henry, 1996). Targeting sequences range in size from about 30 to 120 amino acids and are enriched in hydroxylated residues and deficient in acidic residues (Figure 1). N-proximal 10-15 residues are devoid of Gly, Pro and charged residues, a variable middle region of the transit peptide is rich in Ser, Thr, Lys and Arg, lacking acidic residues; and a carboxy-proximal region is loosely conserved (Ile/Val-x-Ala/Cys-Ala) and is 
the site where proteolitic processing occurs (Von Heijne et al., 1989, De Boer and Weisbeek, 1991). Although there is no apparent similarity in primary sequence among the transit sequences of different precursor proteins, on the basis of import competition studies and transit sequence swapping experiments it was believed that all preproteins use the same mechanism for envelope translocation (Gray and Row, 1995). However, the existence of distinct import pathways has recently been proposed (Kouranov et al., 1999, Nada and Soll, 2004). After translation on cytosolic ribosomes, a cytosolic protein kinase recognizes transit sequences of chloroplast precursor proteins, and is present only in plant extracts, e.g. wheat germ and pea. The protein kinase phosphorylates, in an ATP-dependent manner, one specific Ser or Thr residue within the stroma-targeting domain of the transit sequence (Waegemann and Soll, 1996). Phosphorylation might act as a kinetic signal for targeting, but the translocation of phosphorylated precursor protein is inhibited (Becker et al., 2005). Dephosphorylation is catalyzed by a protein phosphatase, which is probably localized in the outer envelope, and which is required to allow complete import of the precursor into the stroma.

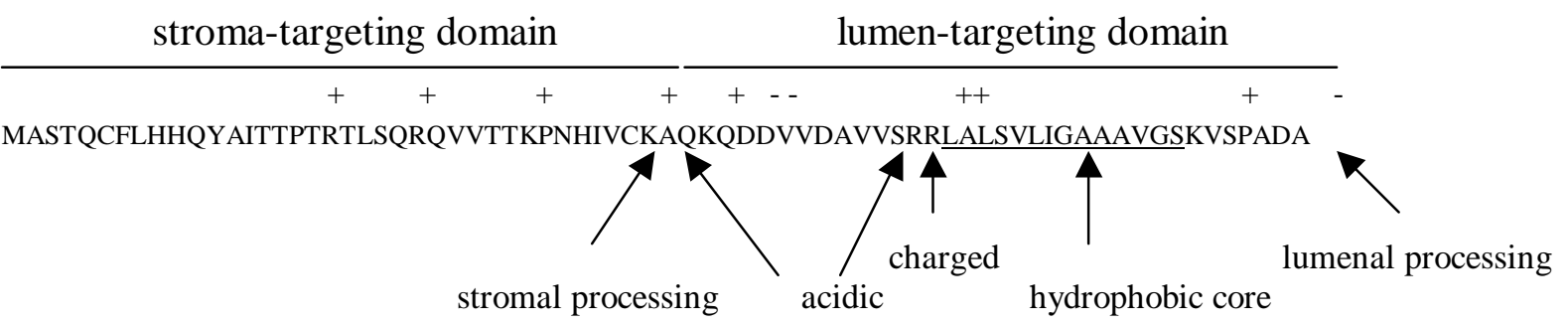

Figure 1. Stroma-targeting domain directs import into chloroplast stroma and lumen-targeting domain to the thylakoid lumen. Stroma-targeting domain is removed by stromal processing peptidase and lumen-targeting domain by a second processing protease (from Cline and Henry, 1996).

Import of precursor proteins into chloroplasts requires protease-sensitive components on the outer envelope membrane (Cline et al., 1985, Friedman and Keegstra, 1989). The lipids found within plastid membranes are also emerging as important players in the targeting, insertion and assembly process of proteins in plastid membranes (Van't Hof et al., 1993, Bruce, 1998, Dörmann and Benning, 2002). The outer chloroplast envelope is strongly negatively charged and enables formation of ionic interactions between preproteins and lipids on the chloroplast surface (Fulgosi and Soll, 2001). Both inner and outer chloroplast envelope membranes are composed of unusual lipids, including monogalactosyldiacylglycerol (MGDG), digalactosyldiacylglycerol (DGDG), sulfolipid sulfoqinovosildiacylglycerol (SQDG) and negatively charged phosphatidylglycerol (PG) (Joyard et al., 1991, Pinnaduwage 
and Bruce, 1996). The lipid/protein ratio of the outer membrane is very high (around 3) (Block et al., 1983) and has a direct influence on protein binding and import across the chloroplast envelopes.

On their way to the inside of the organelle, proteins have to transverse the outer envelope membrane, the intermembrane space and the inner envelope membrane of chloroplasts. The Toc (translocon of the outer envelope membrane) and the Tic (translocon of the inner envelope membrane) translocation complexes are composed of different protein subunits. They are essential for most of the proteins destined for chloroplasts and their interplay enables successful translocation and targeting to the final destination. For the protein translocation inside the chloroplast stroma, formation of contact sites between outer and inner chloroplast membrane, between Toc and Tic complexes, is needed (Pain et al., 1988, Schnell et al., 1990, Alefsen et al., 1994, Kouranov and Schnell, 1996).

To accomplish successful targeting and import into the organelle, freshly synthesized proteins have to remain in the unfolded conformation. Plant 14-3-3 proteins interact specifically with chloroplast precursor proteins, recognizing their phosphorylated transit sequences. The cytosolic protein 14-3-3 is a molecular chaperone that forms a dimer, where each monomer is able to bind one target precursor protein. It is a component of a $200 \mathrm{kDa}$ hetero-oligomeric complex, in which a 14-3-3 dimer cooperates with Hsp70 and perhaps with additional, yet unidentified components (May and Soll, 2000). The formation of the precursor guidance-complex keeps the preprotein in a highly import-competent state. Dissociation of the precursor complex requires ATP. For around $75 \%$ of all plastid precursor proteins at least one site for binding to Hsp70 was predicted (Rial et al., 2000). Chaperones from the Hsp70 family play the important role during protein import into chloroplasts. The chloroplastic outer membrane protein 70 (Com70) is exposed on the cytoplasmic side of the membrane (Ko et al., 1992, Wu et al., 1994). The other, Hsp70 import-associated protein, faces the intermembrane space between the outer and inner envelope membranes (Marshall et al., 1990, Waegemann and Soll, 1991, Schnell et al., 1994).

Multiple pathways exist for targeting and insertion of proteins into the envelope. The first subgroup of proteins destined for the outer chloroplast envelope has internal noncleavable targeting signals and appears to insert directly into the outer envelope membrane (Salomon et al., 1990, Li et al., 1991, Soll et al., 1992). In this group belong single $\alpha$-helical transmembrane proteins Oep7, Omp24, Oep14 and Toc34 (Salomon et al., 1990, Fischer et al., 1994, Li et al., 1991, Seedorf et al., 1995) and pore-forming proteins Oep16, Oep21 and Oep24 (Pohlmeyer et al., 1997, Bölter et al., 1999, Pohlmeyer et al., 1998). Those proteins do 
no require protease-sensitive receptors on the organellar surface or ATP for the integration into the membrane (Waegemann and Soll, 1995). Toc34, for example, neither contains a cleavable presequence, nor uses the general import pathway. It inserts direcly by its Cterminus into the lipid bilayer, stimulated by GTP, and independent of the presence of charged lipids (May and Soll, 1998, Qbadou et al., 2003).

The second subgroup of $\mathrm{OE}$ destined proteins has a cleavable presequence and is represented by Toc75 (Tranel et al., 1995) that needs protease-senstive receptors on the chloroplast surface and ATP hydrolysis for the insertion into the outer envelope membrane. The precursor of Toc75 has a bipartite targeting sequence which N-proxymal part directs the protein to the chloroplast stroma, where it is processed by the stromal processing peptidase to the intermediate-sized form (Tranel et al., 1995, Tranel and Keegstra, 1996, Inoue et al., 2001). The second part of the targeting sequence contains a unique polyglycine stretch that appears to function as a stop transfer domain and is cleaved by plastidic type I signal peptidase (Inoue et al., 2005), creating a mature Toc75 that is subsequently inserted to the outer envelope membrane. For other outer envelope proteins, like Toc159 and Toc64, the topology is not determined so far (Becker et al., 2005).

Most inner envelope proteins are synthesized with cleavable transit peptides and use the general import pathway (Toc and Tic complex, Figure 2) for their translocation. It was suggested that some preproteins contain a hydrophobic stop-transfer signal in their sequence and are released from the translocon on the level of the inner envelope. Others are first targeted to the stroma by their stroma-targeting presequence, and their processed mature form is subsequently re-exported into the inner envelope membrane, by so-called conservative sorting (Lübeck at al., 1997).

Different classes of preproteins seem to exist, which interact preferentially either with Hsp70/14-3-3 or with Hsp90 chaperones. Toc34 recognizes directly preproteins brought by the guidance complex from the cytosol to the chloroplast surface, and is able to recognize and interact with all presequences. Recently it has been shown that some precursor proteins associate with the stromal chaperone Hsp90. In this case, Toc64 acts as an initial docking site for Hsp90-associated precursor proteins, by interacting with Hsp90, and the subsequent transfer of the preprotein from the preprotein-Hsp90-Toc64 complex to Toc34 occurs (Qbadou et al., 2006). Toc34 in its GTP-bound state acts as the initial receptor by binding with high affinity to the transit peptide (Svesnikova et al., 2000, Schleiff et al., 2002, Becker et al., 2004b). Toc34 is converted to its GDP-bound state by preprotein-stimulated GTP hydrolysis (Jelic et al., 2002), resulting in a transfer of the preprotein to Toc159-GTP. 
Following dephosphorylation, the preprotein is driven across the outer membrane through the Toc75 channel via a GTP-dependent Toc159 motor in a sewing-machine-like mechanism (Schleiff et al., 2003a). The transfer of the preprotein from Toc34 to Toc159 might be facilitated by the formation of a heterodimer (Smith et al., 2002). Toc159 is the major phosphoprotein in the outer envelope and essential for chloroplast biogenesis (Bauer et al., 2000). It possesses high homology to Toc34 in its GTPase domain (Kessler et al., 1994, Seedorf at al., 1995). The receptors Toc159 and Toc34 are reset to their GTP-bound state and are ready for further recognition and translocation cycles (Kessler and Schnell, 2006). The preprotein is further transfered to the Toc75 translocation channel. It was proposed that Toc75 has a cytosolic preprotein-binding site and itself is able to differentiate between transit peptides on the base of conformational and electrostatic interactions (Ma et al., 1996, Hinnah et al., 1997, Hinnah et al., 2002). The channel opening and pore diameter are proposed to be controlled by regulatory subunit/s. Toc75 and Toc159 form the minimal translocon unit in vitro that is able to specifically recognize and translocate chloroplast preproteins across a membrane (Schleiff et al., 2003a). Toc34 represents the initial receptor for incoming preproteins and together with Toc159 and Toc75 forms a Toc core complex (Schleiff et al., 2003b).

Toc64, Toc12, intermembrane space Hsp70 and Tic22 associate together to form intermembrane space portion of the translocation complex (Becker et al., 2004a). The J-domain of Toc12 is proposed to recruit the Hsp70 of the outer envelope membrane to the intermembrane space translocon and facilitate its interaction with the preprotein (Becker et al., 2004a).

Seven protein subunits of the inner-envelope translocon are known: Tic110, Tic62, Tic55, Tic40, Tic32, Tic22 and Tic20. Assembly of functional Tic complexes might be dynamic and occurs in response to preprotein translocation (Kouranov et al., 1998). Tic110 was the first identified component of the Tic translocon and represents a major component of active Tic complexes (Kessler and Blobel, 1996, Lübeck et al., 1996). It is an integral inner envelope membrane protein, and structural predictions suggest that it consists of two transmembrane helices at its extreme amino terminus and a 97.5-kDa carboxyl-terminal region that is largely hydrophilic. Tic110 from A. thaliana, atTic110, was shown to be essential for the assembly and function of the protein import machinery of chloroplasts (Inaba et al., 2005). Studies of Tic110 topology and molecular interactions have led to different models for its role in protein import. In the first model, the carboxyl-terminal region of pea Tic110, psTic110, was predicted to extend into the intermembrane space between the outer 


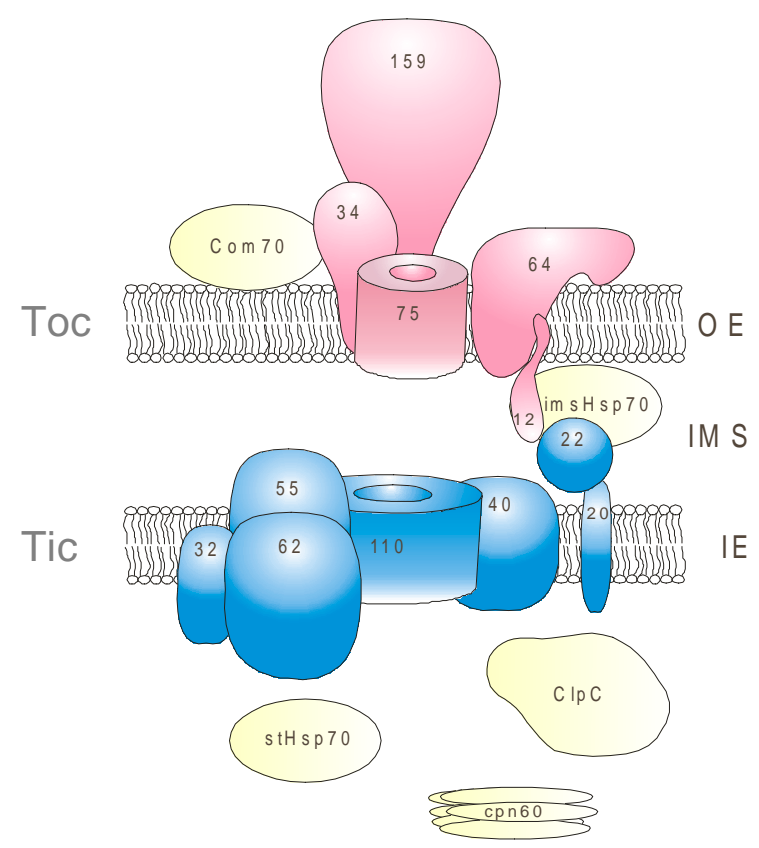

Figure 2. Components of the translocons of the outer (Toc) and the inner (Tic) envelope membrane of chloroplasts. For detailed description see text.

and inner envelope membranes and thereby mediate the interactions between the Toc and Tic complexes during the translocation reaction (Lübeck et al., 1996, Lübeck et al., 1997, May and Soll, 1998). Topology studies performed by Jackson et al. (1998) indicated that psTic110 exists in the opposite orientation with the majority of the protein extending into the stroma. In this model, the large hydrophilic domain of Tic110 was proposed to serve as a docking site for soluble stromal chaperones that assist in the translocation and folding of imported proteins (Kessler and Blobel, 1996, Jackson et al., 1998, Inaba et al., 2003, Chou et al., 2003, Kikuchi et al., 2006). Experiments conducted in our laboratory showed that Tic110 was accessible to proteases from the intermembrane space (Lübeck et al., 1996). Recently, Heins et al. (2002) proposed that Tic110 functions as the protein-conducting channel of the Tic translocon. Tic62 shows strong homologies to $\mathrm{NAD}(\mathrm{H})$ dehydrogenases in eukaryotes and to Ycf39-like proteins present in cyanobacteria and non-green algae and is proposed to regulate protein import into chloroplasts by sensing and reacting to the redox state of the organelle (Küchler et al., 2002). Tic55 possesses a Rieske-type iron-sulphur cluster with a mononuclear ironbinding site (Caliebe et al., 1997) and therefore might act as a regulatory subunit that uses the iron-sulfur cluster as a redox sensor to influence the import competence of the chloroplast (Soll and Tien, 1998). It has been proposed that Tic40 plays an accessory role as a co-chaperone in the stromal chaperone complex that facilitates protein translocation across the inner membrane (Chou et al., 2003, Kovacheva et al., 2005). Tic32 faces the stromal compartment of chloroplasts and is essential for chloroplast viability. It shows homology to 
short-chain dehydrogenases, and its activity is regulated in a $\mathrm{Ca}^{2+} /$ calmodulin dependent manner (Hörmann et al., 2004, Chigri et al., 2006), suggesting a dual role of Tic32 in import: one as a regulatory component that could determine translocation rates across the inner envelope membrane (Chigri et al., 2006) and another as an important subunit in the assembly of the entire complex (Hörmann et al., 2004). For Tic22 it was proposed to serve as a functional link between the translocon complexes in the outer and inner envelopes of chloroplasts (Kouranov and Schnell 1997, Kouranov et al., 1998). Tic20 was shown to participate in the protein import across the inner envelope membrane (Chen et al., 2002) and its role as a part of protein-conducting channel at the inner envelope membrane associated to Tic22 and Tic110 has been proposed (Kouranov et al., 1998, Chen et al., 2000).

In the stroma imported proteins associate with the stromal chaperone Hsp70 and some with the chaperonin Cpn60 (Lubben et al., 1989, Marshall and Keegstra, 1992, Tsugeki and Nishimura, 1993, Madueňo et al., 1993). Pea chloroplasts have at least two stromal Hsp70 isoforms: S78 and CSS1 (Kessler and Blobel, 1996). Cpn60 is a member of the Hsp60 family of chaperones and a homologue of the bacterial chaperone GroEL. It could be co-immunoprecipitated with Tic110 (Kessler and Blobel, 1996) only during contact site formation, suggesting its indirect role in chloroplast protein import. Hsp93 was found to be a component of import complexes regardless of whether precursor proteins were present (Moore and Keegstra, 1993, Akita et al., 1997, Nielsen et al., 1997, Kouranov et al., 1998). Hsp93 and Hsp70 might cooperate in pulling the precursors into chloroplasts in a mechanism analogous to that in mitochondria, while Cpn60 assists the newly imported protein in folding into its native conformation (Jackson-Constan et al., 2001).

In vitro protein import into chloroplasts was first demonstrated by Chua and Schmidt and Highfield and Ellis in 1978, using the precursor of the small subunit of RubisCO. The import process can generally be divided into several steps: binding of the precursor to the receptor proteins on the chloroplast surface, translocation across the two envelope membranes and processing of the precursor by the stroma-localized processing peptidase.

Binding of the precursors to the outer envelope membrane constitutes the first step and produces the first stable intermediate, so-called early import intermediate (Theg and Scott 1993, Schnell and Blobel, 1993). Binding requires less than $50 \mu \mathrm{M}$ NTPs (both ATP and GTP, Soll and Schleiff, 2004) in the cytoplasm or intermembrane space (Olsen et al., 1989, Olsen and Keegstra, 1992, Kessler and al., 1994) and the presence of outer envelope proteins (Olsen and Keegstra, 1992, Theg and Scott, 1993). GTP hydrolysis is necessary for the formation of early-import intermediates, but not for precursor translocation (Young et al., 
1999). Early intermediates are irreversibly bound (Theg and Scott, 1993) and are frequently partially protected from protease treatment of chloroplasts (Friedman and Keegstra, 1989, Waegemann and Soll, 1991). For precursor transport across the outer envelope membrane ATP in the intermembrane space is required (Scott and Theg, 1996) and import through the inner envelope membrane into the stroma progresses if the ATP concentration is raised to around $1 \mathrm{mM}$ (Pain and Blobel, 1987, Theg et al., 1989, Theg and Scott, 1993, Schnell and Blobel, 1993). This ATP is probably needed for the action of molecular chaperones in the stroma, which provide the driving force to complete import into the organelle (Kessler and Blobel, 1996, Nielsen et al., 1997). Upon entering the stromal compartment, the transit sequence is removed by SPP (Robinson and Ellis, 1984, Abad et al., 1989, VanderVere et al., 1995, Richter and Lamppa, 1999).

\subsection{Aim of this work}

\subsubsection{Inner envelope membrane protein Tic110}

Tic110 is synthesized with an N-terminal extension of 37 amino acids which functions as classical transit peptide and engages the general import pathway (Lübeck et al., 1996, Lübeck et al., 1997, Jackson et al., 1998). Targeting to the inner envelope membrane appears to require signals within the transmembrane regions of the protein. Studies using chimeric proteins suggest that Tic110 might use a stromal intermediate during its targeting to the inner membrane (Lübeck et al., 1997). A hybrid protein consisting of the transit sequence of SSU, the N-proxymal part of mature Tic110 and the mature SSU (pSSU-Tic110N-mSSU) is completely imported into the chloroplast stroma. N-terminally processed soluble Tic110N-mSSU sorting intermediate then enters a re-export pathway (Lübeck et al., 1997). Furthermore, dominant negative mutants of Tic110 that disrupt Tic complex formation result in the accumulation of normal Tic110 in the stroma (Inaba et al., 2005). These data suggest that at least a subset of inner membrane proteins is re-inserted into the membrane from the stroma after import. Aim of this work was to investigate import properties of Tic110.

\subsubsection{Intermembrane space protein Tic22}

The pathway of targeting to the intermembrane space of chloroplasts has not been intensively studied yet, due to the lack of a known marker for this subcompartment. Tic22 is nuclearendoded and synthesized as a preprotein with a 50-amino acids long $\mathrm{N}$-terminal presequence. 
Tic22 was identified as a candidate for a component of the general protein import machinery by its ability to covalently crosslink to nuclear-encoded preproteins trapped at an intermediate stage in import across the envelope (Kouranov and Schnell, 1997). Tic22 is a 22-kD protein that is peripherally associated with the outer face of the inner envelope membrane and to a lesser extent to the inner face of the outer envelope membrane (Kouranov et al., 1998, Kouranov et al., 1999).

Kouranov et al. (1999) investigated the import pathway of Tic22 into isolated chloroplasts to define the requirements for targeting of proteins to the intermembrane space. The analysis of deletion mutants and chimeric proteins indicated that the presequence of Tic22 was necessary and sufficient for targeting to the intermembrane space. Import of pTic22 was found to be stimulated by ATP and required the presence of protease-sensitive components on the chloroplast surface. The competition experiments using excess of pSSU indicated that its targeting to the intermembrane space does not involve the general import pathway utilized by stromal preproteins. Kouranov et al. (1999) concluded that the presequence of pTic22 does not function as a stromal transit sequence and that pTic22 is targeted to the intermembrane space of chloroplasts by a novel import pathway that is distinct from known pathways that target proteins to other chloroplast subcompartments. Import properties of Tic22 have been further studied here, aiming to the better characterization of its pathway to the intermembrane space.

\subsubsection{Intermembrane space protein MGD1}

Galactolipids represent more than $80 \%$ of membrane lipids in higher plants, eukaryotic algae and cyanobacteria. 50\% of galactolipid content represents MGDG, a monolayer forming, major structural lipid of chloroplasts and non-green plastids and the most abundant membrane lipid in nature (Gounaris and Barber, 1983, Ohta et al., 2000). It is found in plastid envelopes, as well as in thylakoid membranes. $20 \%$ of polar lipids in plants represents DGDG, a bilayerforming galactolipid. The ratio between MGDG and DGDG is important for chloroplast ultrastructure, especially during response to stress conditions.

Galactolipids are built from glycerol backbone, two long fatty acid chains, and galactose, digalactose or sulfoquinovose unit. They are not charged at physiological $\mathrm{pH}$ and thus represent the only neutral membrane lipid class in thylakoids. Galactolipids are synthesized exclusively at the chloroplast envelopes and from there transported to the thylakoids and extraplastidic membranes (Bruce, 1998, Dörmann and Benning, 2002). UDP-galactose serves as a water-soluble donor of galactose unit to the hydrophobic receptor diacylglycerol, DAG, 
in a reaction catalyzed by MGDG synthase (Ongun and Mudd, 1968). MGDG synthase belongs to a heterogenous family (Maréchal et al., 2000) consisting of type A (atMGD1 from Arabidopsis, csMGD1 from Cucumis sativa, soMGD1 from Spinacia oleracea), type B (atMGD2) and type C (atMGD3) proteins. Type A MGDG synthases are expressed in all tissues, found associated in most cases with the inner envelope and represent the most important MGDG synthase in green tissues (Jarvis et al., 2000). Type B is expressed in flowers and type $\mathrm{C}$ in roots and young leaves (Kobayashi et al., 2004). Both B and C are found to be associated with the outer envelope membrane (Awai et al., 2001). Despite the high abundance of MGDG in plastidic membranes, MGDG synthase represents only 1/1000 of membrane proteins (Joyard et al., 1998), which makes its purification, as well as most of enzymatic analyses, almost impossible (Maréchal et al., 1994). It is a basic protein with an isoelectrical point of 9.5 , active as a $45-48 \mathrm{kDa}$ homodimer and is proposed to be weakly attached to the envelope membranes by electrostatic interactions (Shimojima et al., 1997).

The localization of MGDG synthase activity within envelope membranes is still a matter of controversy and seems to be different in 16:3 (Arabidopsis, potato, tobacco, rape, spinach) and 18:3 (pea, cucumber, barley, maize) plants (Miège et al., 1999, Jarvis et al., 2000, Ohta et al., 2000, Awai et al., 2001). In spinach, the activity was found essentially in envelope fractions enriched in the inner membrane (Tietje and Heins, 1998), whereas the situation is less clear in 18:3 plants. For instance, in pea chloroplasts, Cline and Keegstra (1983) localized the activity to the outer envelope membrane, but in several other analyses both inner and outer membranes seemed to contain significant MGDG synthase activity (Tietje and Heinz, 1998). MGDG synthase is nuclear-encoded in higher plants and no homology with eukaryotic glycosyltransferases has been found in database searches (Ohta et al., 2000). MGDG synthase is very well enzymatically characterized (Maréchal et al., 1994, Maréchal et al., 1995), but its import into chloroplasts has not been investigated yet. It has been proposed to be located in the intermembrane space of chloroplasts, associated with either outer or inner chloroplast envelope. The difference in localization between 18:3 and 16:3 plants was proposed and a lot of questions concearning its topology remained unanswered. That was the reason to investigate topology of MGDG synthase in P. sativum and import properties of A. thaliana atMGD1. AtMGD1 is assumed to be located on the outer side of the inner envelope (Benning and Ohta, 2005). Because MGD1 is proposed to be an intermembrane space protein, its import properties should be compared to those of Tic 22 . 


\section{Materials}

\subsection{Chemicals}

All chemicals were purchased from Sigma Aldrich (München, Germany), Roth GmbH \& Co. (Karlsruhe, Germany), Merck (Darmstadt, Germany), Serva Feinbiochemica (Heidelberg, Germany), Fluka Chemie AG (Buchs, Swiss), Biomol Feinchemikalien GmbH (Hamburg, Germany) and Applichem (Darmstadt, Germany).

Nitrocellulose membrane was purchased from Protran (Schleicher\&Schüll, Germany), blotting-papers from Macherey Nagel (Düren, Germany), Ni-NTA Superflow column was from Qiagen (Hilden, Germany), ATP-agarose (A9264) from Sigma and nProtein A-Sepharose CL-4B from Amersham Biosciences (Uppsala, Sweden). Kodak Biomax-MR films were provided by Eastman Kodak Company (Heidelberg, Germany). Fuji film imaging plates were used for imaging analysis. They were provided by Fuji photo film company, Japan.

\subsection{Enzymes and kits}

Restriction enzymes, DNA- and RNA-polymerase, and other nucleic acids modifying enzymes were supplied by Roche (Mannheim, Germany), MBI Fermentas (St. Leon-Rot, Germany), Pharmacia Biotech (Freiburg, Germany) and Sigma. T4-DNA ligase was purchased from Eppendorf (Hamburg, Germany). DSP crosslinker was supplied by Pierce (München, Germany). Thermolysin was supplied by Merck, apyrase and trypsin by Sigma, soybean trypsin inhibitor and hexokinase by Roche, RNase by Amersham Pharmacia Biotech and lysozyme by Serva.

For small scale plasmid DNA isolation FastPlasmidтм Mini (Eppendorf) or mini boiling DNA prep were used. Large scale DNA isolation was performed with Nucleobond AX (MachereyNagel). Purification of DNA-fragments from agarose gels was performed using Nucleospin Extract II (Macherey-Nagel) and purification of PCR products was carried out using QIAquick PCR Purification Kit provided by Qiagen. DNA sequencing was carried out using BigDye Terminator Cycle Sequencing Ready Reaction Kit supplied by Perkin Elmer (Weiterstadt, Germany). In vitro transcription was performed using chemicals from MBI 
Fermentas. In vitro translation was done with Flexi Rabbit Reticulocyte Lysate System or with TNT Coupled Reticulocyte Lysate System, supplied by Promega (Madison, USA).

\subsection{Molecular weight and size markers}

Protein weight standards MW-SDS-70L and MW-SDS-200 from Sigma were used for SDSPAGE. DNA fragments site marker for agarose gels was prepared by EcoRI/HindIII restriction of $\lambda$-phage DNA, provided by MBI Fermentas.

\subsection{Clones}

\subsubsection{Tic110}

Tic110, the component of the translocon of the inner envelope membrane of chloroplasts that was used for this research derives from Pisum sativum (Kessler and Blobel, 1996, Lübeck et al, 1996). The coding sequence is 2991 bp long, from which first 111 bp represent transit sequence of $3.9 \mathrm{kDa}$, responsible for chloroplast targeting, and the remaining $2880 \mathrm{bp}$ make $101.7 \mathrm{kDa}$ mature protein. For the purpose of enhancing the radioactive signal after in vitro translation, additional 6 methionines were cloned at the C-terminal end of the protein. Tic110 sequence was cloned into pET21d vector.

\subsection{2 pSSU-Tic110N-mSSU}

The hybrid construct pSSU-Tic100N-mSSU (Lübeck et al., 1997) contains the presequence of pSSU (small subunit of RubisCO, 1-192 bp), the N-terminal part of Tic110 (112-817 bp of original clone) and the mature part of SSU (175-564 bp of the original clone). Tic110 sequence originates from Pisum sativum and pSSU from Nicotiana sylvestris (locus NSRUBSSU, Acc. No. X53426, Jamet et al., 1991). pS-110N-mSSU construct is 1257 bp long, encoding the $46 \mathrm{kDa}$ protein. For the purpose of enhancing the radioactive signal after in vitro translation, additional 6 methionines were cloned at the C-terminal end of the protein. pS-110N-SSU sequence was cloned into pET21d vector. 


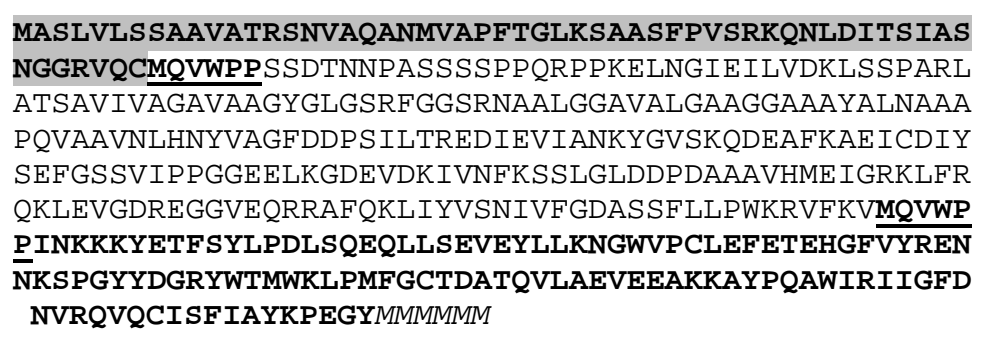

Figure 3. Protein sequence of pS-110N-SSU The presequence and the mature part belonging to pSSU from Nicotiana tabaccum are shown in bold. The middle part of the sequence consists of the N-terminal part of Tic110 (amino acids 38-216). The presequence of the pSSU, as well as of the whole pS-110N-SSU construct, is shown in gray. Underlined are repeating sequences belonging to mSSU. Addition of six methionines at the $\mathrm{C}$-terminus is shown in italic.

\subsection{3 pSSU}

Precursor of the small subunit of RubisCO (pSSU) was used as a control protein for various experiments performed in this thesis. The presequence of this clone originates from soybean Glycine max and mature part from Pisum sativum. The total length of this clone is 537 bp that encode corresponding $19 \mathrm{kDa}$ protein (with $165 \mathrm{bp}$ long presequence). The sequence was cloned into pSP64 vector.

\subsubsection{Tic22}

Tic22/pET21d clone used for this work derives from $A$. thaliana. The sequence used corresponds to the locus At4g33350. $807 \mathrm{bp}$ long coding sequence gives rise to $28.5 \mathrm{kDa}$ big protein, from which $177 \mathrm{bp}$ or $6.3 \mathrm{kDa}$ belong to the chloroplast targeting presequence. For the purpose of import experiments mature form of Tic22 (mTic22, $630 \mathrm{bp}, 22.3 \mathrm{kDa}$ ) and clone with C-terminal deletion (Tic22 $\Delta \mathrm{C}, 675 \mathrm{bp}, 23.9 \mathrm{kDa}$ ) were produced. All 3 clones were used in pET21d or pSP65 vectors.

\subsubsection{MGD1}

U16087 cDNA in pUNI51 vector has been obtained from the Arabidopsis Biological Resource Center. It corresponds to the Arabidopsis locus At4g31780 (UGT81A1), encoding for type A MGDG synthase (AtMGD1). Coding sequence is $1602 \mathrm{bp}$ long, encodes for 533 amino acids, or $56.5 \mathrm{kDa}$ protein. For the purpose of overexpression and in vitro transcription, translation and import experiments, original clone was recloned into pET21d and pSP65 vectors. Also, for the purpose of successful overexpression, N-terminal $321 \mathrm{bp}$ of the preprotein have been removed and MGD1-P clone was created, $1281 \mathrm{bp}$ or $45.3 \mathrm{kDa}$ long. 


\subsubsection{POE33 and $\mathrm{mOE} 33$}

Oxygen evolving complex protein of $33 \mathrm{kDa}$ (pOE33) and its mature form (mOE33) were used for import competition experiments (sequence published by Murata et al., 1987) and the mature form was cloned for the purpose of this study. pOE33 was cloned into pET3c vector and is $990 \mathrm{bp}$ long. mOE33 was cloned into pET21c vector and consists of $747 \mathrm{bp}$.

\subsubsection{Toc34 $\Delta \mathrm{TM}$}

Toc34 $3 \mathrm{TM}$ used for testing the interaction with precursor proteins was from $P$. sativum (Jelic et al., 2002). It contains only $252 \mathrm{~N}$-terminal amino acids, while the C-terminal transmembrane domain is removed.

\subsubsection{Hsp93}

Hsp93/pET21c DNA used for overexpression and antibody production was kindly provided by Prof. Dr. John E. Froehlich, Michigan State University, East Lansing, MI, USA.

\subsection{Primers}

All DNA primers used in PCR reactions were ordered either from MWG-Biotech (Ebersberg, Germany), Qiagen or Invitrogen GmbH (Karlsruhe, Germany).

For cloning of Tic110 into pSP65 vector:

Tic110R 5'-CCC GGG GTC GAC CTA GAA TAC AAA CTT CTC TTC CTC-3’

Tic110L 5'-CCC GGG GAA TTC ATG AAC CCT TCC ACG CTA AAA C-3’’

For adding 6 Met on the C-terminal end of Tic110 and pSSU-Tic110N-mSSU in pET21d vector:

STSpETNcoFor 5'-CCC GGG CCA TGG CTT CCT TAG TTC-3'

Tic110pETNcoFor 5'-CCC GGG CCA TGG ACC CTT CCA C-3'

STSMetXhoRev 5'-CCGG CTC GAG TTA (CAT) 6 GTA GCC TTC GGG CTT GTA-3’

Tic110MetXhoRev 5'-CCGG CTC GAG CTA (CAT) ${ }_{6}$ GAA TAC AAA CTT CTC TTC C-3'

For cloning of Tic22, mTic22 and Tic22 $\Delta \mathrm{C}$ into pET21d vector:

AraTic22NcoFor 5'-CATG CC ATG GAG TCA TCA GTG AAA CCC-3' 
AramTic22NcoFor 5'-CATG CCA TGG ATG CAA TCC AAG TCT GGA ACC CC-3’ AraTic22XhoRev 5'-CATG CTC GAG ACT CTT TGA TCA AAT CCT GC-3' AraTic22 $\Delta$ CXhoRev 5'-CATG CTC GAG AAA TTT GTT GAT CTC CTC TTG ATG-3'

For cloning of Tic22, mTic22 and Tic22 $\Delta \mathrm{C}$ into pSP65 vector: AraTic22pSP65SalFor 5'-CTAG GTC GAC ATG GAG TCA TCA GTG AAA CCC-3' AraTic22pSP65SalRev 5'-CTAG CTG CAG TTA CTC TTT GAT CAA ATC CTG C-3' AramTic22pSP65SalFor 5'-CTAG GTC GAC ATG CAA TCC AAG TCT GGA ACC CC-3' AraTic22 $\Delta$ CpSP65PstRev 5'-CTAG CTG CAG TTA AAT TTG TTG ATC TCC TCT GAT G-3'

For recloning of MGD1 and MGD1-P from pUNI51 to pET21d and pSP65 vectors: AtMGD121NheFor 5'-CCC GGG GCT AGC ATG CAA ACC CTT CAA CGG-3' AtMGD121SacRev 5'-CCC GGG GAG CTC GGC AGT GCA AGA GAG TTG-3’ AtMGD1-P21NheFor 5'-CCC GGG GCT AGC GTC GGA TTA TCG AGT GAT G-3' AtMGD165SacFor 5'-CCC GGG GAG CTC ATG CAA ACC CTT CAA CGG-3' AtMGD165PstRev 5'-CCC GGG CTG CAG TTA GGC AGT GCA AGA GAG-3'

For cloning of mOE33 from pOE33 in pET21c vector into pET21d vector: mOE33NcoFor 5'-CTAG CC ATG GAA GGT GCT CCA AAG AG-3' mOE33XhoRev 5'-GGTG CTC GAG TTC AAG C-3'

\subsection{Vectors}

\begin{tabular}{|l|l|l|l|}
\hline Purpose & Vector name & Company & Reference \\
\hline Translation vectors & pSP64 & Promega & Melton et al., 1984 \\
\cline { 2 - 4 } & pSP65 & Promega & Melton et al., 1984 \\
\hline $\begin{array}{l}\text { Overexpression } \\
\text { vectors }\end{array}$ & pET-21c(+) & $\begin{array}{l}\text { Novagen } \\
\text { (Madison, USA) }\end{array}$ & Studier and Moffat, 1986 \\
\cline { 2 - 4 } & pET-21d(+) & $\begin{array}{l}\text { Novagen } \\
\text { (Madison, USA) }\end{array}$ & Studier and Moffat, 1986 \\
\hline
\end{tabular}




\subsection{E. coli strains}

\begin{tabular}{|l|l|l|l|}
\hline Strain name & Purpose & Company & Author \\
\hline DH5 $\alpha$ & Cloning & GibcoBRL (Eggenstein, Germany) & Woodcock et al., 1989 \\
\hline BL21(DE3) & Overexpression & Novagen (Madison, USA) & Studier and Moffat, 1986 \\
\hline $\begin{array}{l}\text { BL21(DE3) } \\
\text { pRosetta }\end{array}$ & Overexpression & Novagen (Madison, USA) & Studier and Moffat, 1986 \\
\hline
\end{tabular}

\subsection{Growth media}

For the purpose of overexpression of proteins and growth of transformed bacteria for the cloning all E. coli strains were grown in LB medium (Sambrook et al., 1989).

\subsection{Radioisotopes}

${ }^{35}$ S-Methionine/Cysteine mixture and ${ }^{35}$ S-Cysteine with specific activity of $1000 \mathrm{Ci} / \mathrm{mmol}$ were provided from Amersham Biosciences (Freiburg, Germany).

\subsection{Antibodies}

Primary polyclonal antibodies ( $\alpha$-Toc159, $\alpha$-Toc75(III), $\alpha$-Toc75(V), $\alpha$-Toc34, $\alpha$-Tic110, $\alpha$-Tic22 and $\alpha$-OEP16) were generated in the laboratory of Prof. Dr. Jürgen Soll by injection of purified antigens into a rabbit. $\alpha$-Cpn60 antibody was kindly provided by Prof. Dr. Ulrich Hartl vom Max-Planck-Institut für Biochemie (München, Germany). Antibodies $\alpha$-MGD1-P, $\alpha$-Hsp93 and $\alpha$-Hsp70 were produced for the purpose of this thesis (see Methods) by Pineda Antibody Service (Berlin, Germany). Secondary antibodies (goat anti-rabbit alkaline phosphatase conjugate) were obtained from Sigma. 


\subsection{Plant material and growth conditions}

Pisum sativum (sort "Arvica", Praha, Czech Republik) was grown on vermiculit or on sand under $12 \mathrm{~h}$ day / $12 \mathrm{~h}$ night cycle in a climate chamber, at $20^{\circ} \mathrm{C}$.

Spinacea oleracea was bought on the local market, kept on cold and in the dark until use. 


\section{Methods}

\subsection{General molecular biology methods}

\subsubsection{Standard methods}

Bacterial strain culturing and preparation of glycerol stocks were performed according to standard protocols (Sambrook et al., 1989). Competent cells for DNA transformation were prepared according to Chung et al. (1989). Transformation of bacterial DH5 $\alpha$ and BL21(DE3) strains was performed according to Pope and Kent (1996).

\subsubsection{Plasmid DNA isolation}

Isolation of plasmid DNA from $3 \mathrm{ml}$ culture for restriction analysis, subcloning, retransformation into another bacterial strain and sequence analysis were all adapted from the methods of Holmes and Quigley (1981). Large amounts of DNA for in vitro transcription and translation were isolated from $200 \mathrm{ml}$ bacterial cultures by NucleobondAX kit supplied by Macherey-Nagel, according to the manufacturer's instructions. For isolation of high purityDNA for in vitro transcription $\mathrm{CsCl}$ density gradient separation (equilibrium centrifugation based on the different intercalation efficiency of ethidium-bromide) was used (Sambrook et al., 1989). Fast purification of restricted plasmid DNA was performed by phenol/chloroform DNA extraction followed by ethanol or isopropanol precipitation (Sambrook et al., 1989) or by QiaQuick PCR Purification Kit from Qiagen.

\subsubsection{Polymerase chain reaction (PCR)}

The restriction sites for cloning of DNA fragments into plasmid vectors were added by the polymerase chain reaction (Saiki et al., 1998). A standard PCR reaction was carried out as recommended by the polymerase supplier (TripleMaster PCR System, Eppendorf, Hamburg, Germany). Temperatures were adjusted corresponding to the annealing temperatures of the primers. The recombinant PCR technique (Higuchi, 1990) was used in creating the DNA coding for chimerical protein constructs. Fragments of the constructs were synthesized separately in standard PCR reactions, purified over agarose gels by QiaQuick PCR Purification Kit and used as templates for the second round of PCR with the two outer primers, resulting in complete recombinant constructs (Sambrook et al., 1989). Vectors were 
dephosphorylated using alkaline phosphatase from calf intestine (Roche Diagnostics GmbH, Mannheim, Germany), prior to ligation with corresponding inserts.

\subsubsection{Cloning techniques}

Plasmid DNA isolation, restriction of plasmid DNA and PCR-amplified fragments, ligation, as well as agarose gel electrophoresis of DNA were performed according to standard procedures (Sambrook et al., 1989). The reaction conditions for the enzymes were adjusted according to the protocols provided by the manufacturers. Standard techniques were applied for the ligation of Tic22, mTic22, Tic22 $\Delta \mathrm{C}$ and MGD1 into pET21d and pSP65 vectors, and MGD1-P, as well as Tic110 and pS-110N-SSU with additional six C-terminal methionines into pET21d vector.

\subsubsection{In vitro transcription and translation}

For in vitro transcription, isolated plasmid DNA was first purified either on $\mathrm{CsCl}$ gradient and precipitated with isopropanol, or the combination of DNA-isolation kit NucleobondAX and PCR-purification kit was used. The final pellet was resuspended in RNase free water, or the last elution was performed in water or $0.1 \%$ TAE buffer. In vitro transcription of linearized plasmids was carried out in a reaction volume of $50 \mu \mathrm{l}$ containing transcription buffer (supplied by MBI Fermentas), $10 \mathrm{mM}$ DTT, $100 \mathrm{U}$ RNase inhibitor, 0.05\% (w/v) BSA, 0.5 mM ATP, CTP and UTP, $0.375 \mathrm{mM} \mathrm{m}^{7}$-Guanosine (5') ppp (5') Guanosine (cap), 10 U SP6 or T7 RNA polymerase and 2.5-3.0 $\mu \mathrm{g}$ linearized plasmid DNA. The reaction mixture was incubated for 30 minutes at $37^{\circ} \mathrm{C}$ to yield RNA with cap at the $5^{\prime}$-end. Finally, $1.2 \mathrm{mM}$ GTP was added and transcription mixture was incubated for another 2 hours. mRNA was either used directly for in vitro translation or stored under liquid $\mathrm{N}_{2}$. In vitro translation was carried out using the Flexi Rabbit Reticulocyte Lysate System or the TNT Coupled Reticulocyte Lysate System, following the manufacturer's instructions, with optimal RNA concentration and adjusted potassium acetate, magnesium acetate and DTT concentrations, which were determined by test translations. $143 \mu \mathrm{Ci}$ of ${ }^{35} \mathrm{~S}$-methionine/cysteine mixture or $100 \mu \mathrm{Ci}$ of $>90 \%$ pure ${ }^{35} \mathrm{~S}$-cysteine (for the translation of Tic22) were added for radioactive labeling. After translation, the reaction mixture was centrifuged at 50,000xg for 20 minutes at $4^{\circ} \mathrm{C}$ and the postribosomal supernatant was used for import experiments.

Tic110 clone in pET21d vector (T7 polymerase) failed to translate successfully and that was the reason for recloning of Tic110 cDNA into a pSP65 vector, under the promoter of SP6 polymerase. Subsequent optimization did not result in any improvement in final intensity of 
radioactively labelled translation product. Finally, six additional methionines at the end of Tic110 coding sequence were added, to enhance the radioactive signal of ${ }^{35} \mathrm{~S}$-labelled methionines after in vitro translation (Figure 3).

\subsection{Isolation of chloroplasts}

\subsubsection{Isolation of intact chloroplasts from pea}

For isolation of intact chloroplasts (Schindler et al., 1987) pea seedlings grown for 9-11 days on vermiculit, under 12/12 hours dark/light cycle were used. All procedures were carried out at $4^{\circ} \mathrm{C}$. About $200 \mathrm{~g}$ of pea leaves were grinded in a kitchen blender in approximately $300 \mathrm{ml}$ isolation medium (330 mM sorbit, $20 \mathrm{mM}$ MOPS, $13 \mathrm{mM}$ Tris, $3 \mathrm{mM} \mathrm{MgCl}_{2}, 0.1 \%$ (w/v) BSA) and filtered through four layers of mull and one layer of gauze (30 $\mu \mathrm{m}$ pore size). The filtrate was centrifuged for 1 minute at 1500xg and the pellet was gently resuspended in about $1 \mathrm{ml}$ wash medium (330 mM sorbit, $50 \mathrm{mM}$ HEPES/KOH, pH 7.6, $3 \mathrm{mM} \mathrm{MgCl}$ ). Intact chloroplasts were reisolated via a discontinuous Percoll gradient of $40 \%$ and $80 \%$ (in $330 \mathrm{mM}$ sorbit, $50 \mathrm{mM} \mathrm{HEPES} / \mathrm{KOH}, \mathrm{pH}$ 7.6) and centrifuged for 5 minutes at 3000xg in a swing out rotor. After centrifugation two green bands of chloroplasts appeared, where the bottom one consisted of intact chloroplasts. This band was taken and washed two times, and finally resuspended in a suitable volume of wash medium. Samples of chloroplasts $(5 \mu l)$ were resolved in $5 \mathrm{ml}$ of $80 \%$ acetone and chlorophyll concentration was estimated by measuring the optical density at three wavelengths against the solvent (Arnon, 1949). Chloroplasts were used for further import experiments.

\subsubsection{Isolation of intact chloroplasts from spinach}

For isolation of intact spinach chloroplasts, plants were bought at the local market few hours before isolation and kept cool and in the dark. Chloroplasts were isolated using the previously described protocol for pea chloroplast isolation.

\subsection{Preparation of inner and outer envelope vesicles}

For isolation of inner and outer envelope vesicles from chloroplasts pea seedlings grown for 9-11 days on sand, under 12/12 hours dark/light cycle, were used. All procedures were carried 
out at $4{ }^{\circ} \mathrm{C}$. A few $\mathrm{kg}$ of pea leaves were grinded in a kitchen blender in 10-15 1 isolation medium (330 mM sorbit, $20 \mathrm{mM}$ MOPS, $13 \mathrm{mM}$ Tris, $0.1 \mathrm{mM} \mathrm{MgCl} 2,0.02 \%$ (w/v) BSA) and filtered through four layers of mull and one layer of gauze $(30 \mu \mathrm{m}$ pore size). The filtrate was centrifuged for 5 minutes at $1500 x g$ and the pellet was gently resuspended with brush and intact chloroplasts were reisolated via a discontinuous Percoll gradient of $40 \%$ and $80 \%$. Intact chloroplasts were washed twice with wash medium (330 $\mathrm{mM}$ sorbit $\mathrm{pH}$ 7.6), homogenized and further treated according to the modification (Waegemann et al., 1992) of the previously described method (Keegstra and Yousif, 1986).

\subsection{Extraction of proteins from envelope vesicles}

To distinguish between integral membrane proteins and soluble or peripheral membrane proteins, envelope vesicles (section 5.3), were pelleted at $265,000 \mathrm{xg}$ for 10 minutes at $4^{\circ} \mathrm{C}$ and resuspended in either $10 \mathrm{mM}$ HEPES/KOH pH 7.6 solution for 30 minutes on ice, or $6 \mathrm{M}$ urea in $10 \mathrm{mM}$ HEPES/KOH pH 7.6, $0.1 \mathrm{M} \mathrm{Na}_{2} \mathrm{CO}_{3} \mathrm{pH} 11.5$ or $1 \mathrm{M} \mathrm{NaCl}$ for 20 minutes at RT, followed by centrifugation at $265,000 x g$ for 10 minutes at $4^{\circ} \mathrm{C}$. Separated membrane and soluble fractions were analyzed by SDS-PAGE and western-blotting.

\subsection{Treatments of chloroplasts before import}

\subsubsection{ATP depletion from chloroplasts and in vitro translation product}

Prior to chloroplast isolation, the peas were at least 1 hour in the dark (mostly over night). After isolation, intact chloroplasts were left on ice in the dark for 30 minutes in order to deplete ATP and therefore allow subsequent import experiments to be influenced only by exogenously added ATP as an energy source. For radioactively labelled in vitro translation product the ATP-hydrolysing enzyme apyrase was used to deplete endogenous ATP. A standard reaction mixture included $10 \mu \mathrm{l}$ of translation product and $0.5 \mathrm{U}$ apyrase. The reaction mixture was incubated at $25^{\circ} \mathrm{C}$ for 15 minutes and then used directly for import experiments. For depleting the ATP from in vitro translation product Micro Bio-Spin Chromatography Columns (Bio-Rad, Hercules, CA, USA) were used as well, according to producer`s recommendation. Reaction of phosphate transfer from ATP to glucose, catalyzed by hexokinase was the third method used for ATP depletion from translation product. 
Supposing that the ATP concentration in reticulocyte lysate fluctuates around $10 \mu \mathrm{M}$, the exact amount of hexokinase needed for the complete removal of ATP could be calculated. Reaction was performed on $30^{\circ} \mathrm{C}$ for $10-15$ minutes and stopped by addition of excess of competitive inhibitor xylose for 5 minutes at $30^{\circ} \mathrm{C}$.

\subsubsection{Protease pretreatment of isolated intact chloroplasts}

Protease treatment of chloroplasts, prior to import of radioactively labelled protein, was carried out using chloroplasts corresponding to $1 \mathrm{mg}$ chlorophyll, $1 \mathrm{mg}$ thermolysin, and 0.5 mM CaCl 2 . Wash medium (330 mM sorbit, $50 \mathrm{mM} \mathrm{HEPES/KOH,} \mathrm{pH}$ 7.6, $3 \mathrm{mM} \mathrm{MgCl}_{2}$ ) was added to the final volume of $1 \mathrm{ml}$ and the reaction was incubated for 30 minutes on ice. To stop the protease action, $5 \mathrm{mM}$ EDTA were added and intact chloroplasts were reisolated via a discontinuous Percoll gradient containing $5 \mathrm{mM}$ EDTA and washed twice as described before.

\subsection{Import Experiments}

\subsubsection{Import of radioactively labelled proteins into intact chloroplasts}

${ }^{35} \mathrm{~S}$-labelled precursor proteins (translation products) in the maximal amount of $10 \%(\mathrm{v} / \mathrm{v})$ in the reaction were mixed with freshly prepared intact pea chloroplasts (equivalent to $15-20 \mu \mathrm{g}$

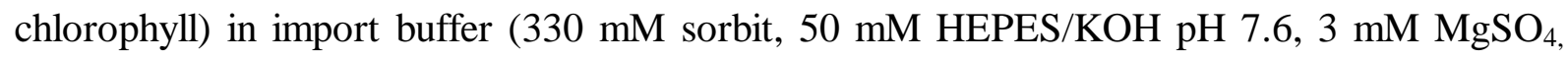
$10 \mathrm{mM}$ Met, $10 \mathrm{mM}$ Cys, $20 \mathrm{mM} \mathrm{K}$-gluconate, $10 \mathrm{mM} \mathrm{NaHCO}_{3}, 2 \%$ BSA (w/v)) and up to 3 mM ATP in a final volume of $100 \mu \mathrm{l}$ (Waegemann and Soll, 1995). Optionally $80 \mathrm{mM} \mathrm{K}_{\mathrm{i}} \mathrm{PO}_{4}$ were added to enhance import rate (Hirsch and Soll, 1995). The import mix was incubated at $25^{\circ} \mathrm{C}$ for up to 20 minutes, depending on experimental requirements. Chloroplasts were reisolated over a 40\% Percoll cushion, washed, and samples were separated by SDS-PAGE. Resulting gels were fluorographed (Bonner and Laskey, 1974) if needed, dried and laid on $\mathrm{X}$-ray sensitive films over night.

\subsubsection{Pulse-chase experiments}

During import into chloroplasts it is possible to track changes in localization and quantity of a protein at different times, to the point it reaches its final destination. For this purpose radioactively labelled precursor protein was added to chloroplasts corresponding to $20 \mu \mathrm{g}$ chlorophyll in the import mixture without ATP (see chapter 5.6.1) and to final volume of 
$100 \mu$ l. For MGD1 $80 \mathrm{mM} \mathrm{K} \mathrm{PO}_{4}$ were added to enhance import. Samples prepared this way were incubated for 2 minutes on ice to accomplish the binding to the import receptors on the chloroplast surface. Samples were centrifuged at 1500xg for 1 minute, washed once in the import buffer and the final pellet was resuspended in import buffer containing $3 \mathrm{mM}$ ATP to allow complete import. The import reactions were performed from 0 up to 32 minutes at $25^{\circ} \mathrm{C}$. Reactions were stopped after different times by addition of Laemmli buffer and samples were analyzed by SDS-PAGE.

For chasing the soluble stromal intermediates, Tic110 and pS-110N-SSU were incubated with $20 \mu \mathrm{g}$ chloroplasts in the import mix containing $3 \mathrm{mM}$ ATP for 2 minutes at $25^{\circ} \mathrm{C}$. Chloroplasts were pelleted and resuspended in the new import mix without ATP, containing $0.5 \mu \mathrm{g}$ thermolysin per $\mu \mathrm{g}$ chlorophyll. The reaction was kept on ice for 10 minutes. Afterwards, the chloroplasts were reisolated on a Percoll cushion and resuspended in the import mix in the presence or the absence of $3 \mathrm{mM}$ ATP. Samples were kept for 5 minutes on ice and imported for 10 minutes at $25^{\circ} \mathrm{C}$. All described steps were performed in the dark to minimize the generation of internal ATP, produced inside chloroplasts via photophosphorylation process. After import reaction, chloroplasts were pelleted $1 \mathrm{~min}$ at $1500 \mathrm{xg}$, washed and separated into soluble and membrane fractions (section 5.6.2.1).

For the purpose of tracking the energy requirement of Tic110 and pS-110N-SSU on reexport import pathway radioactively labelled precursors were incubated with $20 \mu \mathrm{g}$ chloroplasts in the import mix containing $3 \mathrm{mM}$ ATP for 2 minutes at $25^{\circ} \mathrm{C}$. Afterwards chloroplasts were pelleted and resuspended in the new import mix containing either ATP, GTP or PEP at the final concentration of $3 \mathrm{mM}$, or non-hydrolyzable ATP homologs AMPPNP and/or ATP- $\gamma-S$, at the final concentration of $5 \mathrm{mM}$. Fresh import mix was incubated on ice for 5 minutes and subsequent 10 minutes on $25^{\circ} \mathrm{C}$. All incubations were performed in the dark to diminish the influence of internally produced ATP. Afterwards, chloroplasts were pelleted $1 \mathrm{~min}$ at 1500xg, washed, and separated into soluble and membrane fractions.

\subsubsection{Competition with POE33 and MOE33 proteins}

Up to $10 \mu \mathrm{M}$ of purified competitor protein pOE33, as well as its mature form mOE33 (see section 5.10.3) were added to the import mixture. The import experiment was performed as described in section 5.6.1. Maximum $15 \mu \mathrm{g}$ of chlorophyll per reaction was used and the import reaction lasted 5 (pSSU) to 10 or 12 minutes (Tic22, MGD1) at $25^{\circ} \mathrm{C}$. 


\subsubsection{Competition for import by the cytosolic domain of Toc34 receptor GTPase}

In addition to $3 \mathrm{mM}$ ATP and $3 \mathrm{mM}$ GTP, up to $10 \mu \mathrm{M}$ Toc $34 \Delta \mathrm{TM}$ were added to the import mixture. First, Toc34 3 TM was preincubated with GTP in the import mixture for 10 minutes on ice. Subsequently, radioactively labelled translation product has been added for another 10 minutes to allow the interaction of preprotein with Toc34 $\Delta \mathrm{TM}$. At the end, $15 \mu \mathrm{g}$ of chloroplasts were added for import and the reaction was performed for $10-12$ minutes at $25^{\circ} \mathrm{C}$ for Tic22 and MGD1 and 5 minutes for pSSU.

\subsubsection{Inhibition of import by $\mathrm{Ni}^{2+}$ ions}

For the purpose of stopping the import reaction on the level of the outer envelope binding $1 \mathrm{mM} \mathrm{NiSO}_{4}$ was added to the import mix (Rothen et al., 1997). $\mathrm{Ni}^{2+}$ ions interact with the His-tag on the C-terminus of radioactively translated proteins and inhibit their passage across the outer envelope. The alternative to allow only binding, but not the import of preproteins into chloroplasts, was the incubation of the import mixture for 5 minutes on ice.

\subsubsection{Protease posttreatment of intact chloroplasts}

After import, the progress of translocation of proteins through the outer chloroplast envelope was controlled by the treatment of intact organelles with the protease thermolysin. Chloroplasts were pelleted from the import reaction at $1500 \mathrm{xg}$ for 1 minute at $4{ }^{\circ} \mathrm{C}$ and resuspended in $100 \mu \mathrm{l}$ digestion buffer (330 mM sorbit, $50 \mathrm{mM} \mathrm{HEPES} / \mathrm{KOH}, \mathrm{pH}$ 7.6, 0.5 $\left.\mathrm{mM} \mathrm{CaCl}_{2}\right)$. The digestion started with the addition of thermolysin $(0.5 \mu \mathrm{g}$ per $\mu \mathrm{g}$ chlorophyll) and was incubated on ice for 20 minutes. The reaction was stopped by addition of $5 \mathrm{mM}$ EDTA, chloroplasts were pelleted again and washed in the digestion buffer containing $5 \mathrm{mM}$ EDTA.

\subsubsection{Suborganellar localization of imported constructs}

\subsubsection{Chloroplast fractionation into soluble and insoluble fractions}

To distinguish between integral membrane proteins and soluble or peripheral membrane proteins, chloroplasts reisolated after import were lysed in $10 \mathrm{mM} \mathrm{HEPES/KOH} \mathrm{pH} 7.6$ for 30 minutes on ice, followed by centrifugation at $265,000 \mathrm{xg}$ for 10 minutes at $4^{\circ} \mathrm{C}$ to separate the membranes from a soluble fraction. 


\subsubsection{Extraction of proteins with $6 \mathrm{M}$ Urea and $0.1 \mathrm{M} \mathrm{Na}_{2} \mathrm{CO}_{3}$ after import}

After import, reisolated chloroplast were lysed (see section 5.6.2.1), pelleted at 256,000xg, and the pellet was subsequently treated with $6 \mathrm{M}$ urea in $10 \mathrm{mM} \mathrm{HEPES} / \mathrm{KOH} \mathrm{pH} 7.6$ or $0.1 \mathrm{M} \mathrm{Na}_{2} \mathrm{CO}_{3} \mathrm{pH} 11.5$ for 20 minutes at RT. Samples were centrifuged at 256,000xg for 10 minutes at $4^{\circ} \mathrm{C}$ and the pellet and soluble fractions were analyzed by SDS-PAGE.

\subsubsection{Chemical crosslinking and immunoprecipitation}

After import, chloroplasts were re-isolated on a Percoll cushion, washed and chemical crosslinking was performed by incubation of chloroplasts with $0.5 \mathrm{mM}$ dithiobis-succinimdylproprionate (DSP) in $330 \mathrm{mM}$ sorbit, $50 \mathrm{mM}$ HEPES/KOH pH 7.6, $0.5 \mathrm{mM} \mathrm{CaCl}$, for 15 minutes at $4^{\circ} \mathrm{C}$. The reaction was stopped by the addition of $125 \mathrm{mM}$ glycin and further incubation at $4^{\circ} \mathrm{C}$ for 15 minutes. Chloroplasts were washed twice in $330 \mathrm{mM}$ sorbit, $50 \mathrm{mM}$ HEPES/KOH pH 7.6, $0.5 \mathrm{mM} \mathrm{CaCl}_{2}$ and finally lysed in hypotonic buffer $(20 \mathrm{mM}$ HEPES/KOH pH 7.6, $5 \mathrm{mM}$ EDTA) for 30 minutes on ice. A total membrane fraction was recovered by centrifugation at 256,000xg for 30 minutes. Membranes were solublized in $1 \%$ SDS (w/v), $25 \mathrm{mM}$ HEPES/KOH pH 7.6, $150 \mathrm{mM} \mathrm{NaCl}$, diluted tenfold in the above buffer in the absence of SDS, centrifuged for 2 minutes at 20,000xg and the supernatant was used for immunoprecipitation with the antisera against Toc75(III), Toc75(V), Toc34, Tic110 and OEP16. Antisera for the previously indicated proteins were incubated with membranes and $0.5 \%$ egg albumine, rotating for 1 hour at RT, followed by purification on Protein ASepharose. The affinity matrix was washed 3 times with 10 bead-volumes of the mentioned buffer before the elution with Laemmli sample buffer in the presence of $\beta$-mercaptoethanol to split the crosslink products.

\subsection{Binding of Toc34 $\Delta \mathrm{TM}$ to precursor proteins}

For one reaction $300 \mu \mathrm{g}$ purified Toc34 $\Delta \mathrm{TM}$ protein was coupled to $10 \mu \mathrm{Ni}$ NTA matrix in the binding buffer ( $50 \mathrm{mM} \mathrm{NaCl}, 50 \mathrm{mM} \mathrm{Na} \mathrm{PO}_{4}, 0.5 \%$ BSA, $\mathrm{pH} 7.9$ ) for 45 minutes, rotating at RT. Such matrix was used for investigation of interaction of soluble Toc $34 \Delta \mathrm{TM}$ with precursor proteins. The prepared matrix was preincubated with $1 \mathrm{mM} \mathrm{GTP,} \mathrm{and} \mathrm{subsequently}$ 10-12 $\mu 1$ of a radioactively labelled translation product were applied in the reaction containing $1 \mathrm{mM}$ GTP, $2 \mathrm{mM} \mathrm{MgCl}$, $20 \mathrm{mM}$ Tris/ $\mathrm{HCl} \mathrm{pH}$ 7.6, $50 \mathrm{mM} \mathrm{NaCl}$ and 0.5\% BSA. Incubation lasted 45-50 minutes, rotating at RT. The matrix was subsequently washed 3 times with wash 
buffer ( $50 \mathrm{mM} \mathrm{NaCl}, 50 \mathrm{mM} \mathrm{NaPi}, 30 \mathrm{mM}$ imidazole, $\mathrm{pH}$ 7.9) and eluted in $50 \mu$ l elution buffer $(50 \mathrm{mM} \mathrm{NaCl}, 50 \mathrm{mM} \mathrm{NaPi}, 300 \mathrm{mM}$ imidazole, $\mathrm{pH}$ 7.9). The flow through after binding of precursor proteins to Ni-NTA-Toc3 $3 \Delta \mathrm{TM}$ matrix, the last wash and the elution were analysed by SDS-PAGE and the gel was exposed on an x-ray film.

\subsection{Stromal processing assay}

Chloroplasts were isolated from 9-11 days old pea, as described (section 5.2.1). As isolation and wash medium $330 \mathrm{mM}$ sorbit and $50 \mathrm{mM}$ HEPES/KOH pH 7.5 were used. Chloroplasts corresponding to $800 \mu \mathrm{g}$ chlorophyll were pelleted at $1500 \mathrm{xg}$ for 1 minute and lysed in $1 \mathrm{ml}$ of $5 \mathrm{mM}$ ice-cold HEPES/KOH pH 8 for 30 minutes on ice. After centrifugation at 16,000xg for $10 \mathrm{~min}$ on $4^{\circ} \mathrm{C}$, subsequent ultracentrifugation of the supernatant using $137,000 \mathrm{xg}$ for 1 hour was applied. The supernatant containing the active stromal processing peptidase was used in the processing assay. $15 \mu \mathrm{l}$ supernatant, $2.5 \mu \mathrm{g}$ chloramphenicol, $2-3 \mu 1$ radioactively labelled translation product, and $20 \mathrm{mM}$ HEPES/KOH pH 8 were mixed in a total volume of $25 \mu \mathrm{l}$ for 90 minutes at $26^{\circ} \mathrm{C}$. The reaction was stopped by addition of Laemmli buffer and samples were analyzed by SDS-PAGE.

\subsection{Isolation of stromal protein Hsp70}

Intact chloroplast from two Percoll gradients (isolated as described in section 5.2.1) were washed 2 times in $330 \mathrm{mM}$ sorbit, $50 \mathrm{mM}$ Hepes/ $\mathrm{KOH} \mathrm{pH} 7.6,3 \mathrm{mM} \mathrm{MgCl}$, pelleted at $1000 \mathrm{xg}$ for 1 minute, gently resuspended in $15 \mathrm{ml}$ buffer $\mathrm{A}$ (10 mM KAc, $2 \mathrm{mM} \mathrm{MgCl} 2,2$ mM DTT, $20 \mathrm{mM}$ HEPES/KOH pH 7.6), 1\% Triton X-100 and left for 30 minutes on ice. Subsequent centrifugation at $12,000 \mathrm{xg}$ for $15 \mathrm{~min}$ at $4^{\circ} \mathrm{C}$ provided non-solubilized debris in pellet and the supernatant that was diluted to $50 \mathrm{ml}$ with buffer A and coupled to $65 \mathrm{mg}$ ATPagarose (washed previously with buffer A by vacuum filtering through a nitrocellulose membrane). The incubation was performed over night at $4^{\circ} \mathrm{C}$. Next day the matrix was washed with $10 \mathrm{ml}$ buffer A, $10 \mathrm{ml}$ buffer B (1 M KAc, $2 \mathrm{mM} \mathrm{MgCl} 2,2 \mathrm{mM}$ DTT, $20 \mathrm{mM}$ HEPES/KOH $\mathrm{pH}$ 7.6) and again $10 \mathrm{ml}$ buffer A. 10 times $1 \mathrm{ml}$ elution fractions were collected by applying buffer $\mathrm{E}\left(10 \mathrm{mM}\right.$ ATP, $10 \mathrm{mM} \mathrm{MgCl}_{2}, 2 \mathrm{mM}$ DTT, $20 \mathrm{mM}$ HEPES/KOH pH 7.6, $10 \mathrm{mM} \mathrm{KAc).} \mathrm{The} \mathrm{procedure} \mathrm{was} \mathrm{repeated} \mathrm{until} \mathrm{a} \mathrm{sufficient} \mathrm{amount} \mathrm{of}$ 
highly purified protein fractions was collected. Purified fractions were concentrated using Amicon Microcon centrifugal filter devices (Millipore Corporation, Bedford, MA, USA) and stored in $50 \mathrm{mM} \mathrm{NaCl}, 20 \mathrm{mM}$ Tris $/ \mathrm{HCl} \mathrm{pH} 8.5$ in concentration of $1 \mathrm{mg} / \mathrm{ml}$ in the purpose of antibody production.

\subsection{Protein overexpression and purification}

\subsubsection{Overexpression and purification of $\mathrm{Hsp93}$}

Stromal chaperone Hsp93 DNA in pET21c vector was transformed into BL21 (DE3) pRosetta competent cells. Hsp93 protein was overexpressed in $11 \mathrm{LB}$ medium with $100 \mathrm{mg}$ ampicilin, $100 \mathrm{mg}$ chloramphenicol, $1 \mathrm{mM} \mathrm{MgSO} 4$ and $0.4 \%$ glucose. Cells were grown at $37^{\circ} \mathrm{C}$ till they reached O.D.600 of 0.6, induced with $0.5 \mathrm{mM}$ IPTG and incubated at $30^{\circ} \mathrm{C}$ for 3 to 4 hours. Bacteria were pelleted and the protein was purified under denaturing conditions. Each $500 \mathrm{ml}$ bacterial culture was lysed using $30 \mathrm{ml}$ lysis buffer $(50 \mathrm{mM} \mathrm{NaCl}, 20 \mathrm{mM}$ Tris/ $\mathrm{HCl}, 5 \mathrm{mM}$ $\beta$-MeEtOH, $8 \mathrm{M}$ Urea, $\mathrm{pH} 8.0$ to 8.5 ). Urea was previously deionized using MTO-Dowex Marathon MR-3 beads (Supelco, Bellefonte, PA, USA). Solubilized cells were stirred around 40 minutes, till the solution was translucent. The supernatant taken after centrifugation at 10,000xg for 30 minutes at RT was further filtered and purified using anion-exchange chromatography (ResourceQ and MonoQ column, Aekta, Amersham Biosciences). ResourceQ was used for large scale purification and MonoQ for obtaining highly purified fractions. The isoelectrical point for Hsp93 of 6.55 was determined in silico, so the $\mathrm{pH}$ during purification was adjusted to 8.0-8.5. After binding, protein was washed and eluted from the column by applying the linear gradient ranging from $50 \mathrm{mM}$ to $700 \mathrm{mM} \mathrm{NaCl}$ in Tris/ $\mathrm{HCl}$ $\mathrm{pH}$. The purest protein fractions were obtained by elution with $400-550 \mathrm{mM} \mathrm{NaCl}$. Those elutions were concentrated using MF-Millipore membrane filters with the pore size of 30 $\mathrm{kDa}$, and dissolved in $50 \mathrm{mM} \mathrm{NaCl}$ in $20 \mathrm{mM}$ Tris/ $\mathrm{HCl} \mathrm{pH} \mathrm{8.5,} \mathrm{at} \mathrm{a} \mathrm{concentration} \mathrm{of} 1 \mathrm{mg} / \mathrm{ml}$, for the purpose of antibody production.

\subsubsection{Overexpression and purification of MGD1-P}

Due to the problems with expression of full length MGD1 construct, a form of this protein without presequence (MGD1-P) was used for antibody-production. MGD1-P in pET21d vector was overexpressed in BL21(DE3) pRosetta cells, in $11 \mathrm{LB}$ medium with $100 \mathrm{mg}$ ampicilin, $100 \mathrm{mg}$ chloramphenicol, $1 \mathrm{mM} \mathrm{MgSO}_{4}$ and $0.4 \%$ glucose. Cells were grown at 
$37^{\circ} \mathrm{C}$ till they reached O.D.600 of 0.6, induced with $0.5 \mathrm{mM}$ IPTG and incubated at $30^{\circ} \mathrm{C}$ for 3 hours. Bacteria were pelleted, lysed according to QIAexpressionist (Qiagen) protocol and insoluble protein was purified under denaturing conditions on Ni-NTA matrix. For each 500 $\mathrm{ml}$ bacterial culture $1 \mathrm{ml}$ of Ni-NTA matrix was used. After applying the sample in binding buffer (QIAexpressionist), column was washed in $10 \mathrm{ml} \mathrm{W1}(150 \mathrm{mM} \mathrm{NaCl}, 20 \mathrm{mM}$ Tris/HCl, $6 \mathrm{M}$ urea, $\mathrm{pH} 8), 5 \mathrm{ml} \mathrm{W2} \mathrm{(1} \mathrm{M} \mathrm{NaCl,} 20 \mathrm{mM}$ Tris/HCl, $6 \mathrm{M}$ urea, $\mathrm{pH}$ 8), again $5 \mathrm{ml} \mathrm{W1}$ and eluted with 5 times $1 \mathrm{ml}$ of $150 \mathrm{mM} \mathrm{NaCl}, 20 \mathrm{mM}$ Tris/HCl, $6 \mathrm{M}$ urea, $500 \mathrm{mM}$ imidazole, $\mathrm{pH}$ 8. Purification has been tried using $\mathrm{pH}$ gradient for washing $(\mathrm{pH}$ 6.3) and elution ( $\mathrm{pH} 5.9$ and 4.5). This trial did not result in satisfying results and purifications using HiTrap column and by cation-exchange chromatography using PorousS column have been performed ( ̈̈kta). Because these methods did not provide final high protein purity, subsequent purification by gel elution was applied. After SDS-PAGE, gel was washed shortly in $\mathrm{ddH}_{2} \mathrm{O}$, stained in $0.3 \mathrm{M} \mathrm{CuCl}_{2} \times 2 \mathrm{H}_{2} \mathrm{O}$ or $\mathrm{CuSO}_{4} \times 5 \mathrm{H}_{2} \mathrm{O}$ for 5 minutes, the band of the corresponding size (45.3 kDa) was excised, washed few times in $\mathrm{H}_{2} \mathrm{O}, 3$ x 10 minutes in 250 mM EDTA, $250 \mathrm{mM}$ Tris/ $\mathrm{HCl} \mathrm{pH}$ 9, cutted in small pieces and incubated 45 minutes at $\mathrm{RT}$ in $1 \mathrm{ml}$ soaking buffer (125 mM Tris/HCl pH 6.8, 0.5\% SDS, $1 \mathrm{mM}$ EDTA) and $4 \mathrm{M}$ Urea. Afterwards the gel pieces were transferred to dialysis bags (Roth) and eluted over night using electrical current of $6 \mathrm{~mA}$ in DNA gel-electrophoresis cell filled with Laemmli buffer in addition to $4 \mathrm{M}$ Urea. Elutions were collected, concentrated using MF-Millipore membrane filters with the pore size of $30 \mathrm{kDa}$ and dissolved in $50 \mathrm{mM} \mathrm{NaCl}$ in $20 \mathrm{mM}$ Tris/ $\mathrm{HCl} \mathrm{pH} 8$ at the concentration of $1 \mathrm{mg} / \mathrm{ml}$ for the purpose of antibody production.

\subsubsection{Overexpression and purification of POE33 and mOE33}

Transformed BL21(DE3) competent cells were grown in LB medium containing $100 \mu \mathrm{g} / \mathrm{ml}$

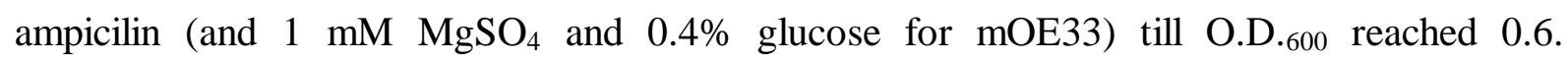
Expression was induced by $1 \mathrm{mM}$ IPTG and cells were grown for 3 hours on $37^{\circ} \mathrm{C}$. Cells were harvested by centrifugation and the expressed protein was isolated in form of inclusion bodies. The protocol for purification of His-tag containing proteins under denaturing conditions and elution by changing the $\mathrm{pH}$ (QIAexpressionist) were applied. Refolding of the protein was accomplished by dialysis against 6, 4, 2 and $0 \mathrm{M}$ urea (over night, for 4 hours, 4 hours and overnight, respectively). Aggregated (misfolded) material was pelleted by centrifugation at $27,000 \mathrm{xg}$ for 10 minutes at $4^{\circ} \mathrm{C}$. The protein concentration in the supernatant was estimated and used for competition experiments. 


\subsubsection{Overexpression and purification of Toc34 $\Delta \mathrm{TM}$}

Toc34 $3 \mathrm{TM}$ protein was overexpressed and purified for the purpose of testing its interaction with precursor proteins prior to import into chloroplasts. The transformed BL21(DE3) cells were grown at $37^{\circ} \mathrm{C}$ in $200 \mathrm{ml} \mathrm{LB}$ medium containing $100 \mu \mathrm{g} / \mathrm{ml}$ ampicilin to O.D. ${ }_{600}=0.6$ and the expression was initiated by the addition of $1 \mathrm{mM}$ IPTG. After three hours of incubation at $37^{\circ} \mathrm{C}$ the cells were harvested by centrifugation and the expressed protein was isolated soluble. The bacterial pellet corresponding to $200 \mathrm{ml}$ liquid culture was lysed in 20 ml lysis buffer (50 mM Na $\mathrm{PO}_{4}, \mathrm{pH} 7.9,300 \mathrm{mM} \mathrm{NaCl}, 10 \mathrm{mM}$ imidazole) in addition to 25 mg lysozyme, and incubated for 30 minutes on ice. Sample was sonicated 6 times 10 bursts at a middle strength, with 10 seconds of cooling period between bursts, and centrifuged at $25,000 x$ for 30 minutes at $4^{\circ} \mathrm{C}$. Supernatant was applied on $400 \mu 1 \mathrm{Ni}-\mathrm{NTA}$ matrix, washed with 15 column volumes of the same buffer containing $30 \mathrm{mM}$ imidazole and eluted by 250-300 mM imidazole. Final protein concentration was determined by the Bio-Rad method. The protein was always used fresh and diluted so that a maximum imidazole concentration in import reaction did not exceed $30 \mathrm{mM}$.

\subsection{Coimmunoprecipitation of Tic110}

Pea chloroplasts corresponding to $200 \mu \mathrm{g}$ chlorophyll were pelleted at 1500xg for 1 minute at $4^{\circ} \mathrm{C}$ and solubilized in $200 \mu \mathrm{l}$ IP buffer (immunoprecipitation buffer consisting of $25 \mathrm{mM}$ HEPES/NaOH pH 7.6 and $150 \mathrm{mM} \mathrm{NaCl}$ ) with addition of $1.5 \%$ DeMa and $0.05 \%$ egg albumine. After solubilization, the sample was diluted to $1 \mathrm{ml}$ with IP buffer, $0.5 \%$ egg albumine and $5 \mu$ l primary antiserum $(\alpha-\mathrm{Hsp} 70, \alpha$-Hsp93) or preimmune serum $(\alpha$-preHsp70, $\alpha$-preHsp93) were added and incubated rotating for $90 \mathrm{~min}$ at RT. $50 \mu \mathrm{l}$ of Protein A-Sepharose matrix was washed in IP buffer containing 0.5\% egg albumine and 0.3\% DeMa, applied to the sample, and incubated rotating for another 90 minutes on RT. Matrix was subsequently washed 2 times with $500 \mu$ IP buffer containing $0.3 \%$ DeMa and 1 time without DeMa. Elution was performed using Laemmli buffer, vortexing and boiling the sample at $99^{\circ} \mathrm{C}$ for 1 minute. Flow through, washes and elution fractions were analyzed by SDS-PAGE and western blotting, using $\alpha$-Tic110 antibodies.

Coimmunoprecipitation of Tic110 and pSSU-Tic110N-mSSU soluble stromal intermediates was performed as follows. After import, intact chloroplasts were reisolated on a $40 \%$ Percoll cushion, washed, fractionated onto soluble and pellet fractions (see section 
5.6.2.1). Soluble fraction was diluted 1:1 in 2xIP buffer and incubated with $5 \mu 1$ of $\alpha$-Hsp70, $\alpha$-Hsp93, $\alpha$-Cpn60 or $\alpha$-OEP16 antisera for 1 hour at RT, in the presence of $0.5 \%$ egg albumine and $0.3 \%$ DeMa. Subsequent purification on Protein A-Speharose was performed as described previously.

\subsection{Methods for separation and identification of proteins}

\subsubsection{SDS-Polyacrylamide-Gel-Electrophoresis (SDS-PAGE)}

Electrophoretic separation of proteins under denaturing conditions was performed in a discontinuous gel system (Laemmli, 1970). For separation gels 10 to $13 \%$ acrylamide was used, and for stacking gels $5 \%$ acrylamide.

\subsubsection{Detection of proteins in gels}

After separation of proteins on polyacrylamide gels a number of standard detection techniques were used. Staining solution containing 0.18\% (w/v) Coomassie Brilliant Blue R250 in 50\% $(\mathrm{v} / \mathrm{v})$ methanol and 7\% (v/v) acetic acid enabled visual recognition of bands equivalent to 0.1-10 $\mu \mathrm{g}$ protein. Gels were stained for 15 minutes on a shaker and unbound dye was removed by $15-30$ minutes washing in a destaining solution $(40 \%(\mathrm{v} / \mathrm{v})$ methanol, $7 \%(\mathrm{v} / \mathrm{v})$ acetic acid, 3\% (v/v) glycerol). For detection of ${ }^{35} \mathrm{~S}$-labelled proteins, acrylamide gels were dried and exposed on x-ray films (Kodak Biomax MR) underlayed with photoenhancing screens (Agfa MR800) for 1-3 days, depending on the amount of radioactivity present. To estimate the radioactivity of the samples, films were laid on imaging plates (BAS-MS) for 1 day. The plates were screened using phosphoimaging scanner FLA-3000 and band intensities were analyzed using AIDA image analyzer program for advanced image analysis (Advanced Image Data Analyzer v.3.52, 2D densitometry).

\subsubsection{Western transfer}

Directly following the SDS-PAGE proteins were transferred onto nitrocellulose membranes using the semi-dry electro blotting technique (Trnovsky, 1992). The gel was laid onto 6 sheets of blotting-papers, soaked in anode buffer I (0.3 M Tris pH 10.4, 20\% MeOH), 4 sheets soaked in anode buffer II (25 mM Tris $\mathrm{pH} 10.4,20 \% \mathrm{MeOH})$, the membrane rinsed in the same buffer and covered by 6 sheets of blotting-papers in cathode buffer ( $25 \mathrm{mM}$ Tris $\mathrm{pH} 7.5$, $4 \mathrm{mM}$ aminocapronic acid and $20 \% \mathrm{MeOH}$ ). The "sandwich" was placed into transfer 
apparatus chamber. The working current was $0.8 \mathrm{~mA}$ per $\mathrm{cm}^{2}$ of the gel for 1 hour. The membranes were then stained with $0.5 \%$ Ponceau $\mathrm{S}$ in $1 \%$ acetic acid, molecular standard marker positions were marked, and the membranes subjected to further analysis.

\subsubsection{Immunodecoration of proteins with antibodies}

The nitrocellulose membrane with bound proteins was saturated by $3 \times 10$ minutes wash in TN (100 mM Tris/HCl pH 7.5, $150 \mathrm{mM} \mathrm{NaCl,} 0.3 \%$ milk powder, $0.03 \%$ BSA) or in TTBS buffer (100 mM Tris/HCl pH 7.5, 0.9\% NaCl, 1\% BSA, 0.1\% Tween-20). The same buffers were used for all following procedures. The membranes were sealed in polyethylene bags and incubated for 3 hours at RT or over night at $4^{\circ} \mathrm{C}$ with the appropriate antiserum, diluted 1:1000. Excess primary antibodies were then removed by $3 \times 10$ minutes washing and the secondary antibody (goat anti-rabbit alkaline phosphatase conjugate) in the dilution 1:10000 was applied to the membrane and incubated for 1 hour. Staining reactions were performed in $100 \mathrm{mM}$ Tris/ $\mathrm{HCl} \mathrm{pH} 9.5,100 \mathrm{mM} \mathrm{NaCl}, 5 \mathrm{mM} \mathrm{MgCl}_{2}, 0.035 \% \mathrm{NBT}$ (w/v) and $0.0175 \%$ $\operatorname{BCIP}(w / v)$.

\subsubsection{General methods of protein biochemistry}

Protein concentrations in solutions were roughly evaluated from the stained gels by comparing protein bands to protein weight standards. More precise determination of protein concentration was performed using the Bradford Bio-Rad reagens. To precipitate proteins from diluted solutions, $10 \%$ final concentration of TCA (w/v) was added to samples and incubated for 20 minutes on ice, followed by 15 minutes centrifugation at 25,000xg. Samples were neutralized by Tris-base, as demanded by experimental conditions.

\subsubsection{Fluorography}

Fluorography was applied for the purpose of enhancing of weak radioactive signals after the SDS-PAGE, before drying the gel and exposing it on an x-ray film. Two methods were used. After destaining, gel was incubated 4 times 10 minutes in DMSO, 30 minutes in 20\% PPO in DMSO, washed 2 times 15 minutes in water prior to drying and exposure on a film.

Another approach used was washing a gel after destaining 2 times for 15 minutes in water, incubating 20 minutes with $16 \%$ (w/v) Na-salicylate and drying (without washing in water). 


\section{Results}

\subsection{Import properties of the inner membrane protein Tic110}

\subsubsection{Import of Tic110 and pSSU-Tic110N-mSSU}

In vitro translation of Tic110 resulted in two products, one around 95 and another around 120 $\mathrm{kDa}$ (Figure 4A, lane 1). Translation of $\mathrm{pS}-110 \mathrm{~N}-\mathrm{SSU}$ resulted in a single band of around 56 $\mathrm{kDa}$ (Figure 4A, lane 3). Both radioactively labelled Tic110 translation products were imported into chloroplasts under the standard conditions (see section 5.6.1) and the lower product probably represents a version of Tic110 truncated at the C-terminus. The difference in size between the precursor and the mature form of the protein corresponded to the transit sequence of $3.9 \mathrm{kDa}$ that was removed by the stromal processing peptidase upon import into chloroplasts (Figure 4A, lane 2). Thermolysin posttreatment of the chloroplasts removed the precursors bound to the organellar surface, leaving only protected, mature forms of proteins intact. Both mature forms of Tic110 were protected from thermolysin degradation, indicating that the mature Tic110 is localized inside chloroplasts (Figure 4A, lane 2). The hybrid construct pS-110N-SSU was imported into pea chloroplasts under the standard conditions as well. Its mature part, lacking the presequence of around $6.5 \mathrm{kDa}$, was protected from thermolysin degradation (Figure 4A, lane 4).

The pulse-chase import experiment was performed to observe the import intermediate forms and to investigate their energy requires. Import of radioactively labelled translation products pTic 110 and pS-110N-SSU has been performed for 2 minutes at $25^{\circ} \mathrm{C}$. Chloroplasts were pelleted and reisolated in the fresh import mix containing thermolysin in the concentration of $0.5 \mu \mathrm{g}$ per $\mu \mathrm{g}$ chlorophyll to remove precursors just bound to the chloroplast surface. Chloroplasts were reisolated and finally incubated in the presence or the absence of $3 \mathrm{mM}$ ATP. The addition of ATP was essential for the complete incorporation of soluble Tic110 and pS-110N-SSU into the membrane. Under limiting ATP conditions, only a portion of the proteins was imported into the inner envelope membrane (Figure $4 \mathrm{~B}$, lanes 2, 7, 11) and mostly around $20 \%$ could be observed in the soluble fraction as soluble stromal intermediates (Figure 4B, lanes 3, 8, 12). In the presence of $3 \mathrm{mM}$ ATP almost the entire mature protein pool was inserted into the membrane (Figure 4B, lanes 5, 10 and 14 in 
comparison to 4, 9 and 13), while the lack of an energy source arrested the soluble intermediate in the stromal compartment. These results indicated that the integration of Tic110 and pS-110N-SSU in the inner envelope is dependent on ATP.

A

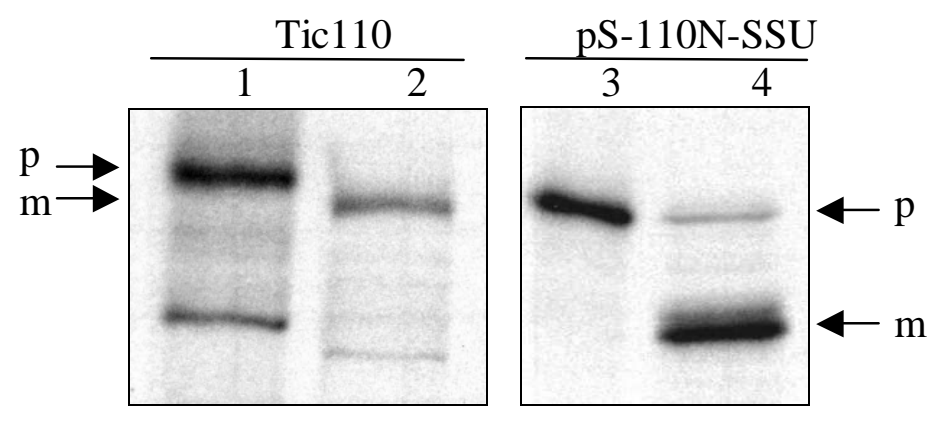

B

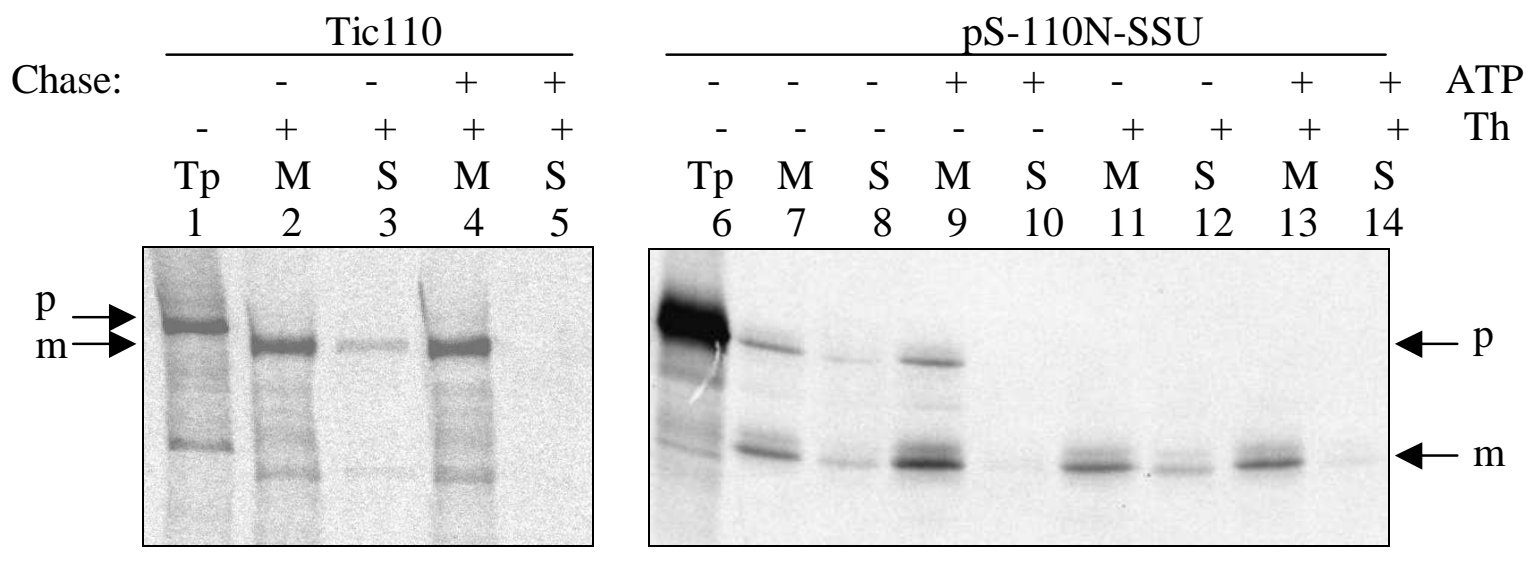

Figure 4. A. Tic110 and pS-110N-SSU import into pea chloroplasts. Import of ${ }^{35} \mathrm{~S}$-pTic110 and ${ }^{35} \mathrm{~S}-\mathrm{pS}-110 \mathrm{~N}-\mathrm{SSU}$ was performed for 15 minutes at $25^{\circ} \mathrm{C}$, in the presence of $3 \mathrm{mM}$ ATP. After import, samples were treated by thermolysin (lanes 2 and 4). Lanes 1 and 3 indicate $1 / 10$ of the translation products. B. Chase of Tic110 and pS-110N-SSU. ${ }^{35}$ S-pTic110 and ${ }^{35} \mathrm{~S}-\mathrm{pS}-110 \mathrm{~N}-\mathrm{SSU}$ were incubated with intact pea chloroplasts for 2 minutes at $25^{\circ} \mathrm{C}$, in the presence of $3 \mathrm{mM}$ ATP (pulse). After this time chloroplasts were transferred to the new import mix either in the presence of $0.5 \mu \mathrm{g}$ thermolysin per $\mu$ g chlorophyll (lanes 2-5, 11-14) or without thermolysin (lanes 7-10) for 10 minutes on ice. After this treatment, chloroplasts were reisolated on a Percoll cushion, washed and resuspended in the import mix containing $3 \mathrm{mM}$ ATP (lanes 4, 5, 9, 10, 13, 14) or without ATP (lanes 2, 3, 7, 8, 11, 12) and incubated for 10 minutes at $25^{\circ} \mathrm{C}$. After import, chloroplasts were separated into the membrane (M) and soluble (S) fractions. Lanes 1 and 6 represent 1/10 of the translation product used for the import reaction. Precursor (p) and mature (m) forms of Tic110 and pS-110N-SSU are indicated by arrows.

The stromal intermediates that were observed for both imported proteins represent the mature forms: Tic110 and 110N-SSU. To confirm that the removal of their transit sequences occurs in the stroma, pTic110 and pS-110N-SSU were subjected to a stromal processing. Chloroplast stroma was isolated (section 5.8) and incubated together with the radioactively labelled 
Tic110 and pS-110N-SSU for 90 minutes at $26^{\circ} \mathrm{C}$. Tic110 and pS-110N-SSU were both processed by the stromal extract (Figure 5).
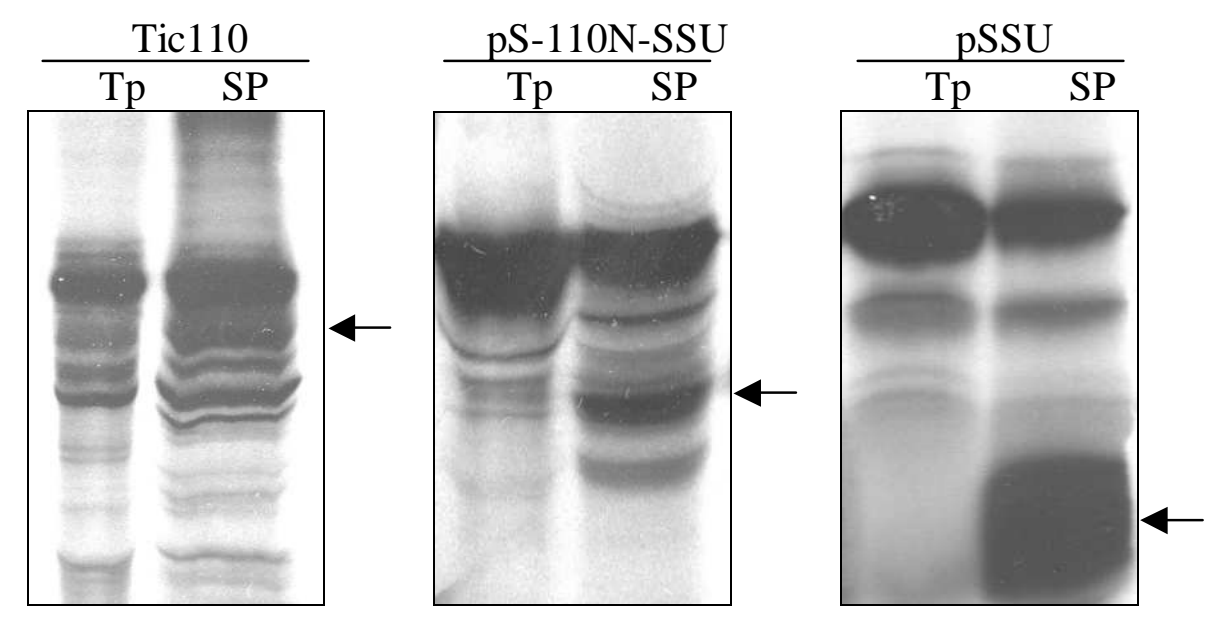

Figure 5. Processing of Tic110 and pS-110N-SSU by the stromal extract. Stroma was isolated from pea chloroplasts and incubated with radioactively labelled translation products ${ }^{35} \mathrm{~S}$-pTic 110 , ${ }^{35} \mathrm{~S}-\mathrm{pS}-110 \mathrm{~N}-\mathrm{SSU}$ and ${ }^{35} \mathrm{~S}-\mathrm{pSSU}$ as a control, for 90 minutes at $26^{\circ} \mathrm{C}$. Reactions were stopped by addition of Laemmli buffer and samples were analyzed by SDS-PAGE. Tp indicates $2 \mu 1$ of the corresponding translation product and SP $2 \mu \mathrm{l}$ of the corresponding translation product after processing. Mature forms: Tic110, 110N-SSU and SSU, appearing after the processing by the stromal processing peptidase, are indicated by arrows.

\subsubsection{Soluble stromal import intermediates}

In order to follow the import of Tic110 through different chloroplast compartments on a time-scale, a standard import reaction was performed in the presence of $3 \mathrm{mM}$ ATP. After $0.5,2,5,10$ and 20 minutes the reaction was stopped by transfer to ice and reisolated chloroplasts were separated into the membrane and soluble fractions (section 5.6.2.1).

Changing the import duration led to the appearance of the stromal intermediate found in the soluble fraction, the amount of which increased during the first five minutes of import (Figure 6, lanes 5, 7,9). After this time the concentration of the soluble intermediate in the stroma decreased (Figure 6, lanes 11, 13) because of its re-export into the membrane. Enrichment of the membrane fraction with mature proteins could be observed with time (Figure 6, lanes 4, 6, 8, 10, 12). The appearance of the soluble intermediate was observed already after 0.5 minutes of import (Figure 6, lane 5) and its ratio to the membrane-bound form increased to reach the maximum value between 2 and 5 minutes (Figure 6, lanes 7, 9). After this time, the soluble stromal intermediate progressively moved to the membrane fraction, and nearly no intermediate was observed after 20 minutes of import (Figure 6, lane 
13). The described experiment enabled us to follow the import pathway of Tic110 through the chloroplast envelopes to the stroma, where its transit peptide was removed by the stromal processing peptidase (Figure 5), to the re-export into the inner envelope, to its final location.

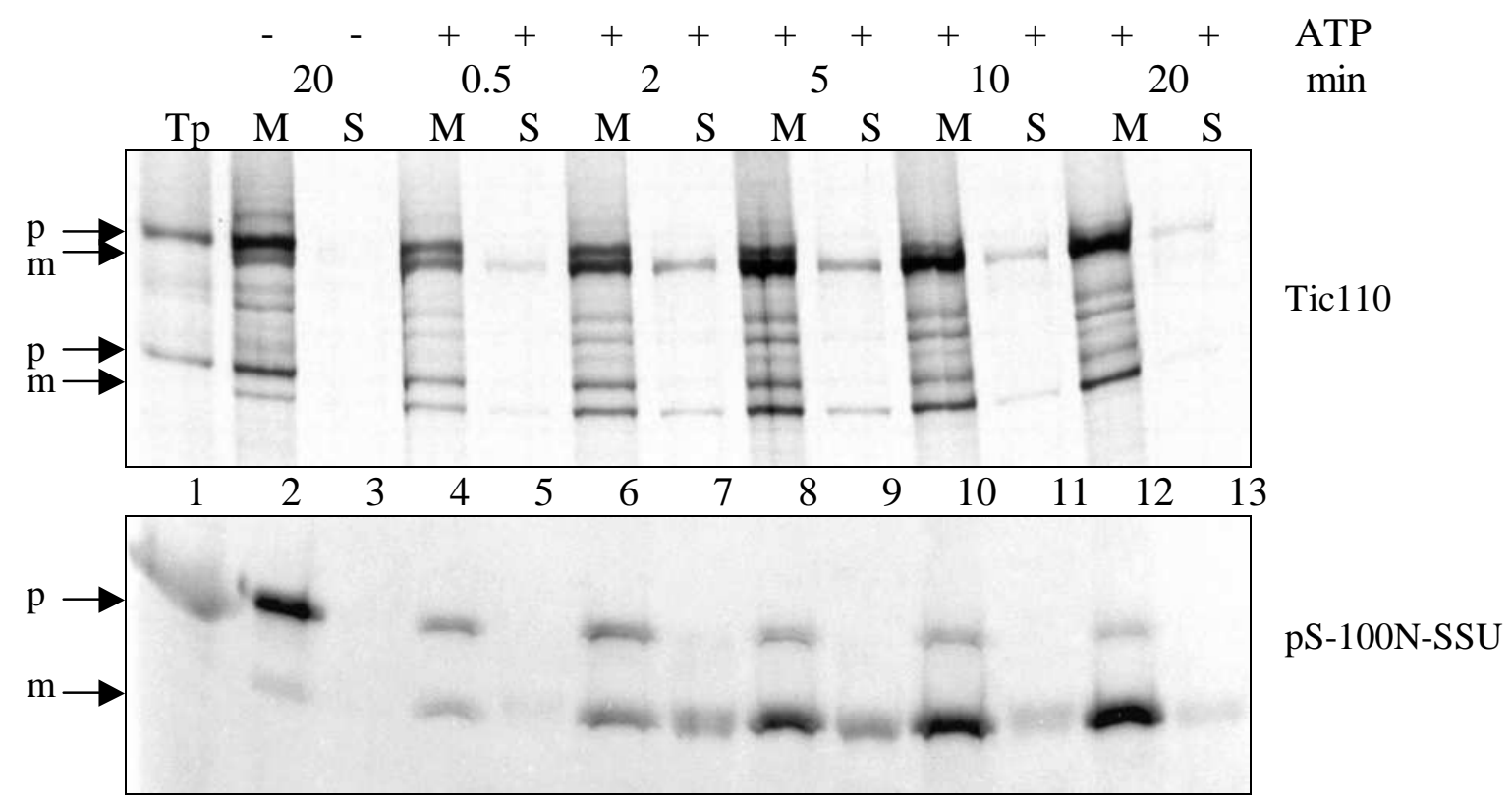

Figure 6. Time-scale import of Tic110 and pS-110N-SSU. ${ }^{35}$ S-pTic110 and ${ }^{35}$ S-pS-110N-SSU were incubated with intact pea chloroplasts using the standard conditions, for $0.5-20$ minutes at $25^{\circ} \mathrm{C} .3 \mathrm{mM}$ ATP (lanes 4-13) or no NTPs (lanes 2-3) were used in the import mix. After import chloroplasts were separated into the membrane (M) and soluble (S) fractions. Lane 1 indicates $1 / 10$ of the translation product used for the import reaction. Precursor (p) and mature (m) forms of Tic110 and pS-110N-SSU are indicated by arrows.

A control experiment without the addition of ATP in the import mixture (Figure 6, lanes 2 and 3) indicates that the import into the stroma needs higher amounts of ATP. Comparing the ratio between soluble and membrane-associated forms of Tic110 on a time-scale, it appears that most of the protein was found in the membrane fraction. These results imply that the processing of Tic110 is a very fast process. The mature Tic110 visible under the ATPdepletion probably represents only the portion of Tic110 that is arrested in the inner envelope membrane translocon, rather than completely translocated to its final position. In such state the protein can still be processed by the stromal processing peptidase, by extruding its $\mathrm{N}$-terminal presequence towards the stroma.

After 2 minutes of import the ratio between the soluble and the membrane-bound form of pS-110N-SSU was around one (Figure 6, lanes 6 and 7). The same ratio for Tic110 never exceeded 0.15 to 0.20 . The import conditions under which the highest soluble to membrane protein ratio was obtained were considered the most suitable for further investigations of the 
energy requirements of Tic110 and 110N-SSU for subsequent re-export from the stroma to the inner membrane. For this purpose, radioactively labelled precursors pTic110 and pS-110N-SSU were incubated with intact pea chloroplasts corresponding to $20 \mu \mathrm{g}$ chlorophyll, in the import mix containing $3 \mathrm{mM}$ ATP for 2 minutes at $25^{\circ} \mathrm{C}$. After 2 minutes chloroplasts were pelleted and resuspended in a new import mix containing ATP, GTP, PEP, or non-hydrolyzable ATP homologs AMP-PNP and ATP- $\gamma$-S, as described in section 5.6.1.1.

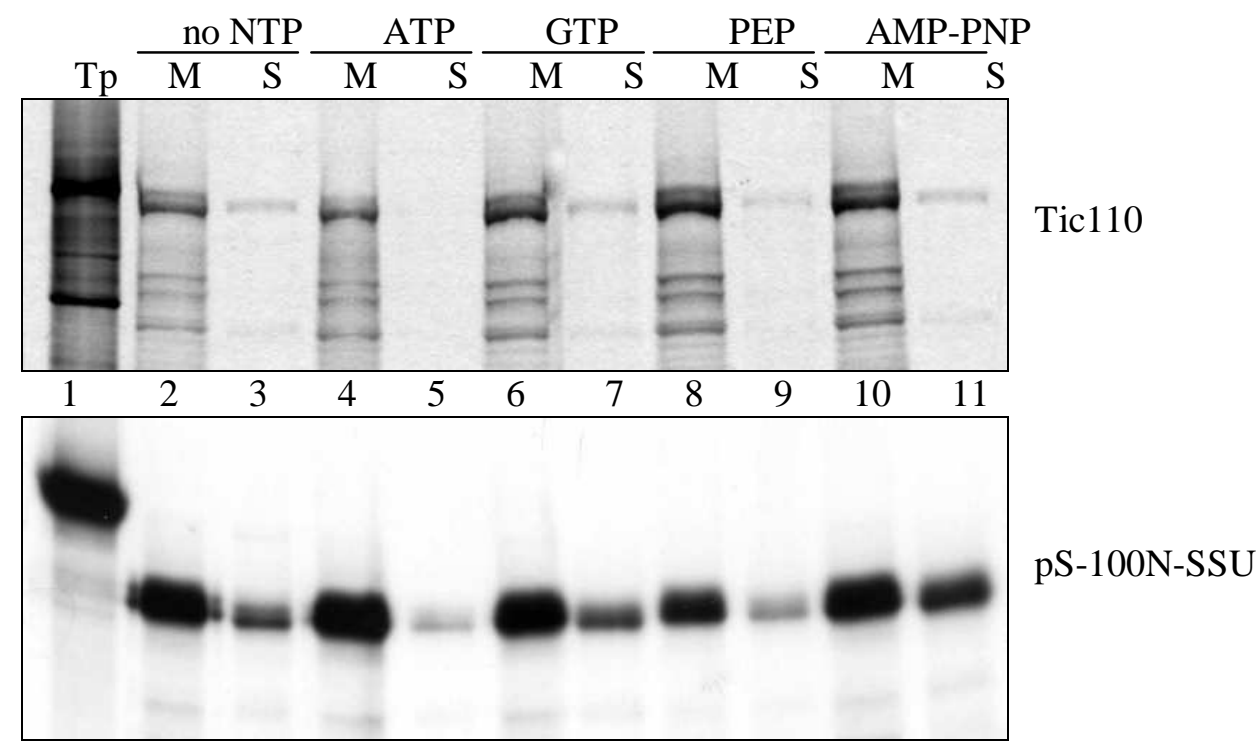

Figure 7. Energy requirement for the re-export of Tic110 and pS-110N-SSU. Both radioactively labeled proteins were imported in the standard import reaction at $25^{\circ} \mathrm{C}$ in the presence of $3 \mathrm{mM}$ ATP. The import was arrested after 2 minutes and the chloroplasts were transferred into a new import mix containing either no NTPs (lanes 2 and 3), $3 \mathrm{mM}$ ATP (lanes 4 and 5), $3 \mathrm{mM} \mathrm{GTP} \mathrm{(lanes} 6$ and 7), 3 $\mathrm{mM}$ PEP (lanes 8 and 9) or $5 \mathrm{mM}$ AMP-PNP (lanes 10-11). Samples were incubated for 5 minutes on ice followed by 10 minutes of import at $25^{\circ} \mathrm{C}$. After import chloroplasts were separated into the membrane (M) and soluble (S) fractions. Lane 1 represents 1/10 of the translation product used for the import reaction.

Using this approach the soluble stromal intermediate, visible after 2 minutes of import (Figure 6, lane 7), could be investigated. After addition of $3 \mathrm{mM} \mathrm{ATP,} \mathrm{all} \mathrm{(Tic110)} \mathrm{or} \mathrm{nearly} \mathrm{all}$ (pS-110N-SSU) soluble intermediates were re-exported to the membrane (Figure 7, lanes 4 and 5), while in the presence of other energy sources the soluble form could be trapped to a certain extent (Figure 7, lanes 3, 7, 9, 11). The highest ratio between the soluble intermediate and the membrane fraction of the protein was observed after addition of GTP and AMP-PNP (Figure 7, lanes 7 and 11 respectively), that was especially obvious for pS-110N-SSU import. ATP- $\gamma-S$ alone or in the combination with AMP-PNP provided the same result as AMP-PNP (data not shown). The observation that GTP and GMP-PNP provided the highest ratio 
between the soluble and the membrane portion of the protein was used for the isolation of larger quantities of soluble stromal intermediates Tic110 and 110N-SSU for the purpose of immunoprecipitation with stromal chaperones (section 5.11). To further improve the quantity of the soluble stromal intermediate, imports were performed under different temperature, time and ATP conditions (data not shown).

Several combinations of conditions resulted in the highest soluble to membrane fraction ratio of Tic110 and $110 \mathrm{~N}-\mathrm{SSU}$. 3.5 minutes of import at $25^{\circ} \mathrm{C}$ in the presence of $3 \mathrm{mM}$ ATP were chosen for further experiments (Figure 8, lane 7). To investigate the interaction of the soluble intermediates with the stromal chaperones first the antibodies against Hsp93 and Hsp70 had to be produced.

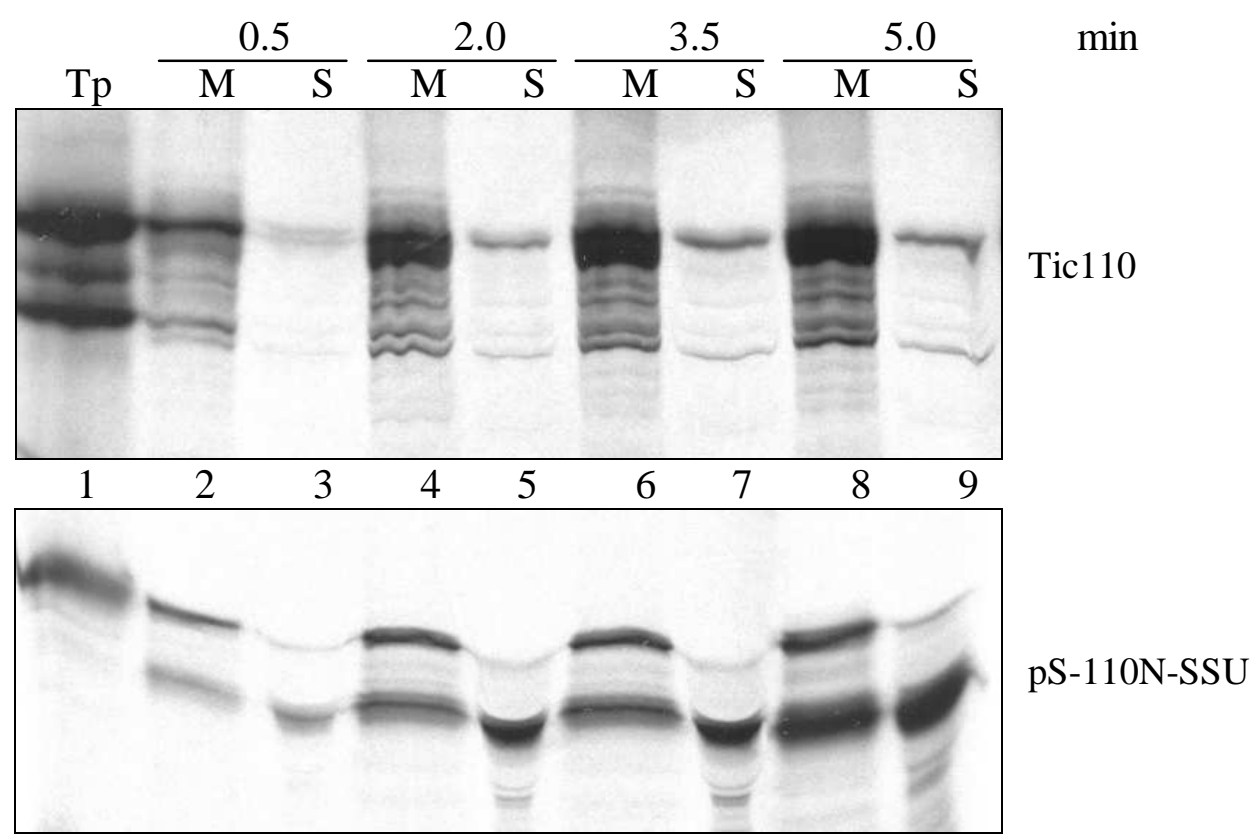

Figure 8. Import conditions used for the isolation of the soluble stromal intermediate. ${ }^{35} \mathrm{~S}-\mathrm{Tic} 110$ and ${ }^{35} \mathrm{~S}-\mathrm{pS}-110 \mathrm{~N}-\mathrm{SSU}$ were incubated with intact pea chloroplasts using standard conditions, for 0.5-5.0 minutes at $25^{\circ} \mathrm{C}$, in the presence of $3 \mathrm{mM}$ ATP. After import chloroplasts were reisolated on a Percoll cushion, washed, and separated into the membrane $(\mathrm{M})$ and soluble $(\mathrm{S})$ fractions. Lane 1 indicates $1 / 10$ of the translation product used for the import reaction.

\subsubsection{Purification of stromal chaperone Hsp70}

Stroma of Pisum sativum contains at least two isoforms of the Hsp70 family: proteins CSS1 and S78 (Kessler and Blobel, 1996). The population of stromal Hsp70s was isolated from pea by direct coupling of freshly isolated chloroplast stroma to an ATP-agarose matrix, as described in section 5.9. The ability of the chaperones to bind ATP was used for their 
purification. Hsp70 was eluted from the ATP-agarose matrix by increased ATP concentration and collected fractions were analyzed by SDS-PAGE (Figure 9A). The resulting protein fraction was highly pure and no further purification was required (Figure 9A, B). The purified protein was concentrated to $1 \mathrm{mg} / \mathrm{ml}$ and used for antibody production. Antibodies were tested on chloroplast stroma and purified protein (Figure 9C).

A

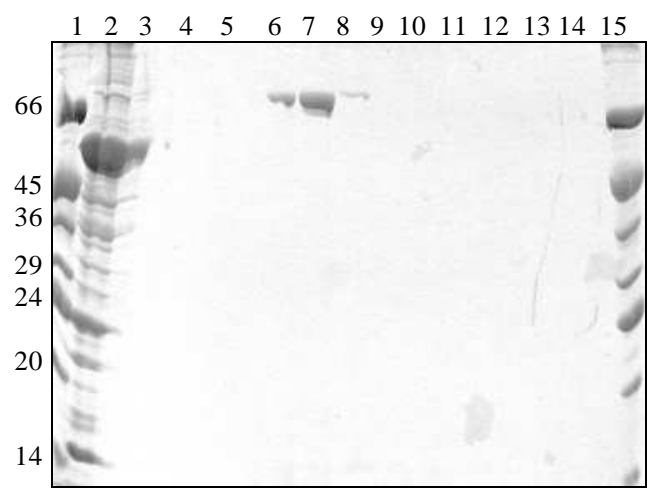

B

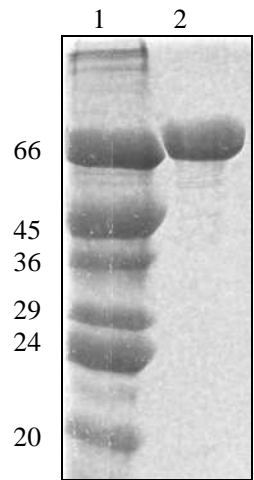

$\mathbf{C}$

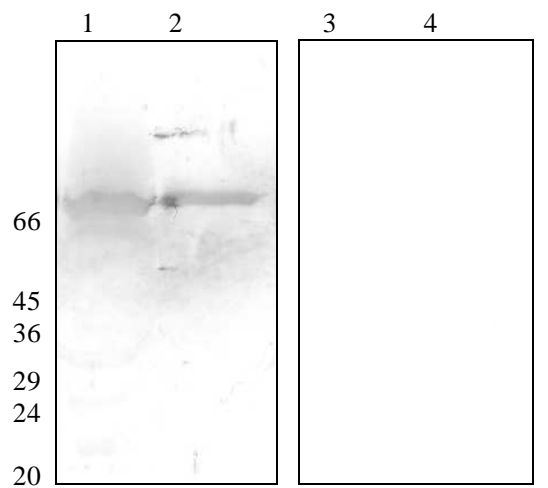

Figure 9. Purification of Hsp70 from chloroplast stroma. A. Freshly isolated stroma from pea chloroplasts was coupled to an ATP-agarose. $20 \mu \mathrm{l}$ of low molecular weight marker is in lanes 1 and $15,1 / 1000$ of the flow through after coupling to the ATP-agarose is in lane 2. Lanes 3-5 represent $0.65 \%$ of washes with buffer A1, buffer B and buffer A2, respectively. Lanes 6-14 represent the elution fractions, 1/20 volume of each. B. Hsp70 combined from elutions 1, 2 and 3 (lanes 6-8 in A) from many purifications was concentrated to $1 \mathrm{mg} / \mathrm{ml}$, using Amicon Microcon centrifugal filter devices (lane 2 contains $5 \mu \mathrm{l}$ ). $20 \mu \mathrm{l}$ of low molecular weight marker is in lane 1. C. Testing of Hsp70 antibodies produced in rabbit. $7.5 \mu \mathrm{l}$ of chloroplast stroma (lanes 1 and 3) and $0.5 \mu \mathrm{g}$ of purified Hsp70 protein (lanes 2 and 4) were analyzed by SDS-PAGE and western transfer and subsequent immunodecoration with $\alpha$-Hsp70 antibodies (lanes 1,2) or with the preimmune serum (lanes 3, 4).

\subsubsection{Overexpression and purification of the stromal chaperone Hsp93}

Hsp93 was overexpressed under conditions described in chapter 5.10.1 (Figure 10A). Overexpressed protein was tested for solubility and its biggest portion was isolated as inclusion bodies (Figure 10B). Overexpressed Hsp93 protein was further purified under denaturing conditions. After solubilization in $8 \mathrm{M}$ Urea, $\mathrm{pH} 8.0$ to 8.5 , the sample was centrifuged and the supernatant filtered and purified using the anion-exchange chromatography ResourceQ and MonoQ columns (Äkta, Amersham Biosciences). Absorption spectrum at $280 \mathrm{~nm}$ was measured and fractions of $0.5 \mathrm{ml}$ corresponding to each observed peak were collected and analyzed by SDS-PAGE (Figure 11A-C). 
A

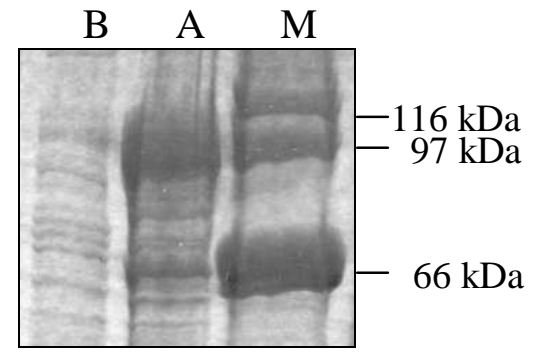

B

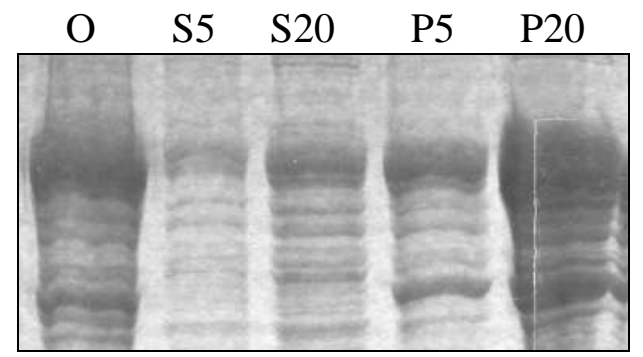

Figure 10. Overexpression of Hsp93. A. Hsp93 was overexpressed in E. coli BL21(DE3) pRosetta competent cells, for 3 hours at $30^{\circ} \mathrm{C}$. B, sample before induction; A, sample after $3 \mathrm{~h}$ of expression, and $\mathrm{M}, 20 \mu \mathrm{l}$ of high molecular weight marker. B. After overexpression, bacteria were lysed and pelleted. The soluble $(\mathrm{S})$ and pellet $(\mathrm{P})$ fractions were analyzed by SDS-PAGE. O, sample after overexpression; S5 and S20 indicate 5 and $20 \mu \mathrm{l}$ of soluble portion of Hsp93; and P5 and P20, 5 and $20 \mu \mathrm{l}$ of non-soluble portion of Hsp93, respectively.

Purified protein was concentrated to $1 \mathrm{mg} / \mathrm{ml}$ and used for antibody production. Antibodies were tested on chloroplast stroma and purified proteins (Figure 11D).

A

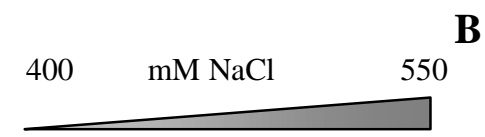

C

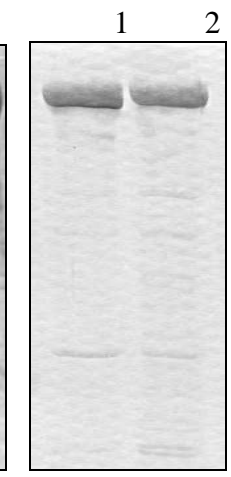

D

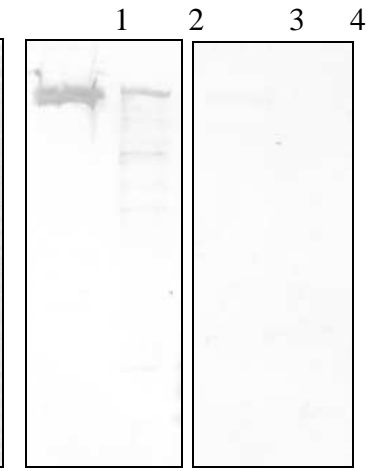

Figure 11. Purification of overexpressed protein Hsp93. A. After solubilization in $8 \mathrm{M}$ urea, Hsp93 was purified using anion-exchange ResourceQ column. The sample was washed with $50 \mathrm{mM} \mathrm{NaCl}$ in Tris/HCl pH 8. 1/10 of the wash fractions was loaded on the gel (lanes 2-4). Elution was performed using the gradient ranging from $50 \mathrm{mM}$ to $700 \mathrm{mM} \mathrm{NaCl}$ in Tris/ $\mathrm{HCl} \mathrm{pH} 8.1 / 10$ of the fractions corresponding to the peak region $(400-550 \mathrm{mM} \mathrm{NaCl}$ ) were analyzed by SDS-PAGE (lanes 5-14). Lane 1 indicates a mixture of high and low molecular weight marker, $10 \mu \mathrm{l}$ each. B. Concentrated sample after purification using ResourceQ column, 1 and $3 \mu 1$ were analyzed by SDS-PAGE (lanes 2 and 3, respectively). Lane 1 indicates $10 \mu \mathrm{l}$ of high molecular weight markers. C. After purification on MonoQ column samples were concentrated using Millipore membrane filters with the pore size of $30 \mathrm{kDa}$ and prepared for antibody production in the final concentration of $1 \mathrm{mg} / \mathrm{ml} .2$ and $1 \mu \mathrm{l}$ were analyzed by SDS-PAGE (lanes 1 and 2, respectively). D. Testing of Hsp93 antibodies produced in rabbit. $7.5 \mu \mathrm{l}$ of chloroplast stroma (lanes 1 and 3) and $0.5 \mu \mathrm{g}$ of purified Hsp93 protein (lanes 2 and 4) were loaded on SDS-PAGE and analyzed by western transfer and subsequent immunodecoration by $\alpha$-Hsp93 antibodies (lanes 1,2 ) or by preimmune serum (lanes 3, 4). 


\subsubsection{Immunoprecipitation of Tic110 by stromal chaperones}

It has been observed that the native psTic110 associates reversibly with the Hsp93 and Cpn60 chaperones of the chloroplast stroma (Nielsen et al., 1997, Kessler and Blobel, 1996). It has been proposed that Tic110 together with Hsp93 and Tic40 most likely functions at a later stage of protein transport across the inner envelope, at the stromal side, by recruiting Hsp93, a proposed ATP-dependent import motor (Chou et al., 2003, Kikuchi et al., 2006). To investigate whether Tic110 interacts with those chaperones during its own import into chloroplasts, for aquiring the native conformation in the stroma, conditions for co-immunoprecipitation of soluble intermediates with stromal chaperones were established.

A

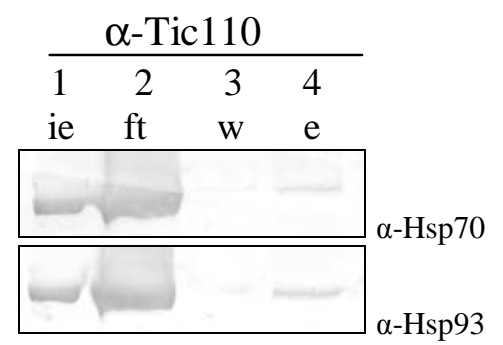

B

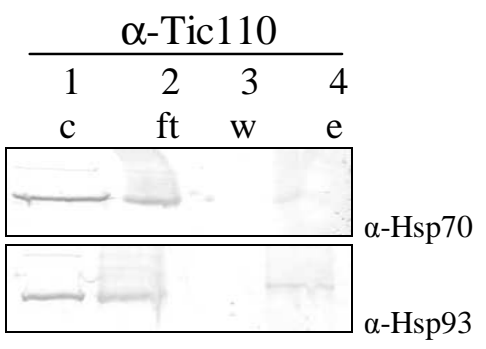

C

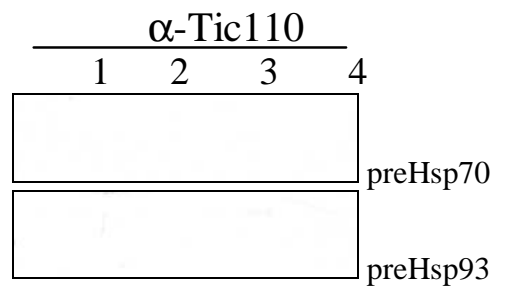

Figure 12. Co-immunoprecipitation of Tic110 by stromal chaperones Hsp93 and Hsp70. A. Inner envelope vesicles corresponding to $20 \mu \mathrm{g}$ protein were solubilized and incubated with $\alpha$-Hsp70 and $\alpha$-Hsp93 antibodies for 90 minutes at RT. Antibodies were bound to ProteinA-Sepharose matrix during the subsequent 90 minutes incubation, rotating at RT. After washing and elution, samples were dissolved in Laemmli buffer and solubilized inner envelope (ie), 1/20 of the flow through after incubation with Protein A-Sepharose ( $\mathrm{ft}$ ), 1/10 from the last wash (w), and the elution (e) were analyzed by SDS-PAGE, western-blotted and immunodecorated with $\alpha$-Tic110 antibodies. B. The same experiment performed using solubilized chloroplasts corresponding to $200 \mu \mathrm{g}$ chlorophyll per reaction. C. In a control reaction with the preimmune serum against Hsp70, as well as Hsp93, chloroplasts (lane 1), inner envelope vesicles (lanes 2 and 3) and egg albumine (lane 4) were tested for interaction specificity and elution fractions analyzed by SDS-PAGE, western transfer and immunodecoration with $\alpha$-Tic110 antibodies.

Intact chloroplasts or inner envelope vesicles were solubilized with $1.5 \%$ DeMa and immunoprecipitation using antibodies raised against stromal chaperones Hsp70 and Hsp93, $\alpha-\mathrm{Hsp} 70$ and $\alpha$-Hsp93, was performed (section 5.11). Antibodies against Hsp70, as well as Hsp93 interacted with Hsp70 and Hsp93 inside the inner envelope vesicles and co-immunoprecipitated Tic110 bound to those chaperones. The size of the bands shown corresponded to the size of Tic110 and was recognized by $\alpha$-Tic110 antibodies (Figure 12A). This observation was confirmed by the analysis of solubilized chloroplasts (Figure 12B). Immunodecoration of preimmune serum control reaction with $\alpha$-Tic110 antibodies did not show any signal, implying that the interactions between Tic110 and chaperones were specific. 
A

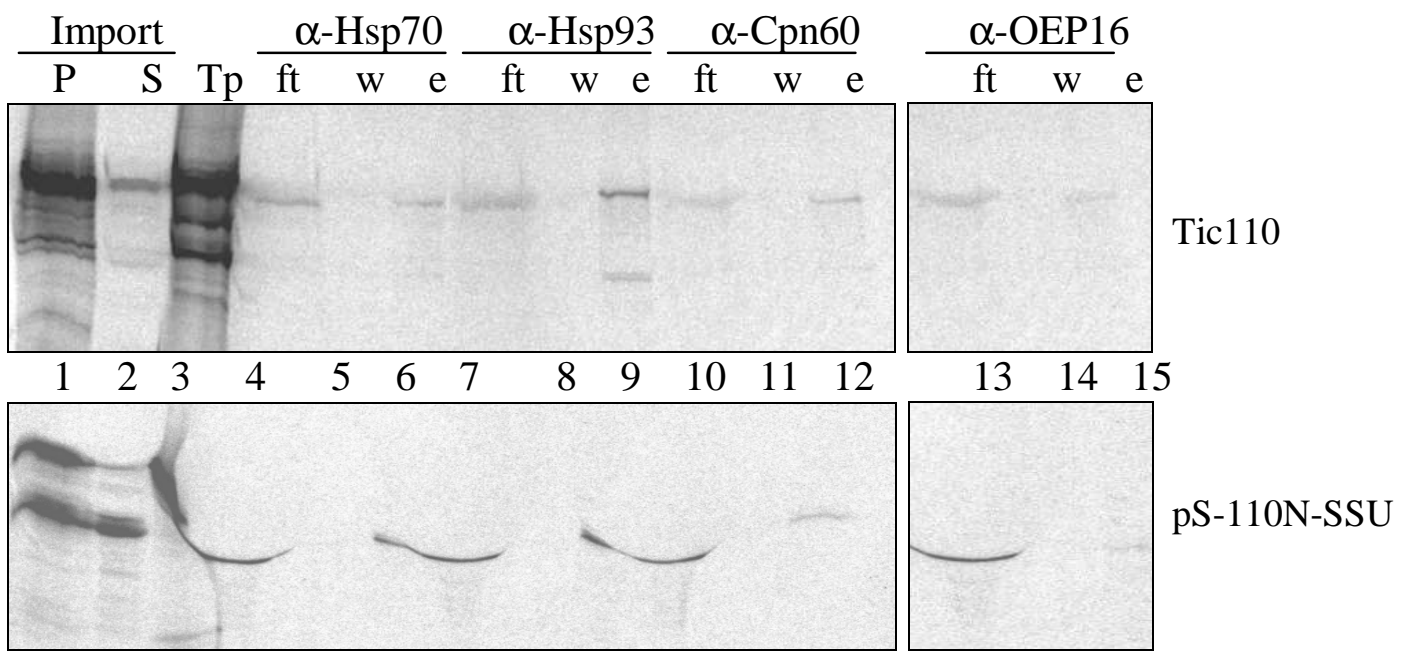

B

Tic110

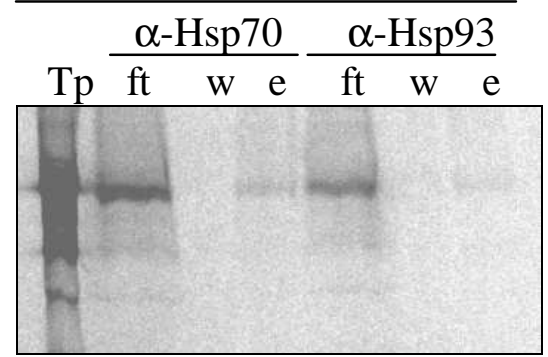

pS-110N-SSU

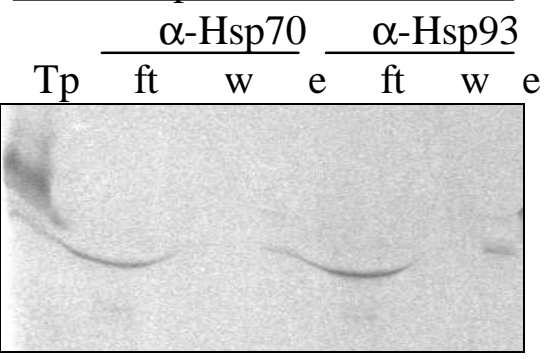

Figure 13. Co-immunoprecipitation of stromal intermediates of Tic110 and 110N-SSU by stromal chaperones. ${ }^{35} \mathrm{~S}$-pTic 110 and ${ }^{35} \mathrm{~S}-\mathrm{pS}-110 \mathrm{~N}-\mathrm{SSU}$ were incubated with intact pea chloroplasts corresponding to $20 \mu \mathrm{g}$ chlorophyll for 3.5 minutes at $25^{\circ} \mathrm{C}$, in the presence of $3 \mathrm{mM}$ ATP. After import, chloroplasts were reisolated on a Percoll cushion, washed and separated into the membrane and soluble fractions. A. The soluble stromal intermediates ${ }^{35} \mathrm{~S}$-Tic110 and ${ }^{35} \mathrm{~S}-110 \mathrm{~N}-\mathrm{SSU}$ were dissolved in IP buffer and incubated with antibodies raised against stromal chaperones: $\alpha$-Hsp70, $\alpha$-Hsp93, $\alpha$-Cpn60 and $\alpha$-OEP16 as a control, $0.5 \%$ egg albumine, and 0.3\% DeMa for 1 hour at RT. Antibodies were collected using Protein A-Sepharose. The flow through after the incubation with Protein A-Sepharose (ft), the third wash (w) and the elution with Laemmli sample buffer (e) were analyzed by SDS-PAGE. The pellet and the supernatant after 3.5 minutes of import were loaded in lanes 1 and 2. Tp indicates $1 / 10$ of the translation product used for each experiment. B. The membrane fractions of ${ }^{35} \mathrm{~S}$-Tic1 10 and ${ }^{35} \mathrm{~S}-110 \mathrm{~N}-\mathrm{SSU}$ were solubilized in $1.5 \%$ DeMa and subsequently dissolved in IP buffer and treated as described in A. Incubation was performed with antibodies raised against stromal chaperones $\alpha$-Hsp70 and $\alpha-H s p 93$.

As described previously (Figure 8), the import time of 3.5 minutes has been selected for the purpose of the isolation of the soluble stromal intermediates of Tic110 and 110N-SSU. Radioactively labelled pTic110 and pS-110N-SSU were incubated with chloroplasts corresponding to $20 \mu \mathrm{g}$ chlorophyll, in a standard import reaction containing $3 \mathrm{mM} \mathrm{ATP}$, for 3.5 minutes at $25^{\circ} \mathrm{C}$. After reisolation and washing steps, chloroplasts were separated into the pellet and soluble fractions. Soluble stromal import intermediates of Tic110 and 110N-SSU were subsequently incubated in immunoprecipitation buffer with antibodies raised against stromal chaperones Hsp70, Hsp93 and Cpn60. Antibodies with bound proteins interacted with 
the Protein A-Sepharose, washed, eluted and analyzed by SDS-PAGE. Soluble Tic110 and $110 \mathrm{~N}-\mathrm{SSU}$ were both immunoprecipitated by all tested antibodies against stromal chaperones. Hsp93 interacted with Tic110 and 110N-SSU to the greatest extent, while the binding to the Hsp70 and Cpn60 was weaker. As a control, binding of the membrane fraction of Tic110 and $110 \mathrm{~N}-\mathrm{SSU}$ to stromal chaperones Hsp70 and Hsp93 was tested. The interaction of stromal chaperones and the mature Tic110 and 110N-SSU incorporated into the inner envelope membrane was very veak. This indicates that Tic110 interacts with stromal chaperones during its own import, before reinsertion into the inner envelope membrane. A slight binding to the control protein OEP16 was ascribed to the background unspecificity (Figure 13, lane 15).

\subsection{Import properties of the chloroplastic intermembrane space proteins Tic22 and MGD1}

To investigate import characteristics of the intermembrane space proteins Tic22 and MGD1, in vitro import experiments have been performed using ${ }^{35} \mathrm{~S}$-labelled precursor proteins, synthesized in the reticulocyte lysate system. It has been reported that Tic 22 can be imported in vitro into pea chloroplasts, though at a very low rate (Kouranov et al., 1999). Tic22 contains a $6.3 \mathrm{kDa}$ long chloroplast-targeting presequence that directs it to its final localization in the intermembrane space. Two homologues of Tic22 were found in A. thaliana genome: At3g23710 and At4g33350. At4g33350 is homologous to P. sativum psTic22 and has been used in this research. Import properties of MGDG synthases have not been investigated yet. Using the ChloroP neural network-based program prediction method (Emmanuelson et al., 1999) chloroplast transit peptides were predicted for the class A MGDG synthases (Figure 14). MGD1 from A. thaliana used in this research belongs to the class A of MGDG synthases and contains a predicted presequence of 106 amino acids (11.2 kDa). Alignments using the ClustalW program (Thompson et al., 1994) showed that the identity between the complete amino acid sequences of atMGD1 and cucumber or spinach MGDG synthases is around $70 \%$ and homology around $90 \%$. By contrast, comparison of the class A MGDG synthases to the atMGD2 and atMGD3 shows much lower homology (around $70 \%$ homology and less than $50 \%$ identity).

Radioactively labelled precursors of Tic22 and MGD1 were incubated with intact chloroplasts in the presence of $3 \mathrm{mM}$ ATP. This concentration of ATP is sufficient to allow 


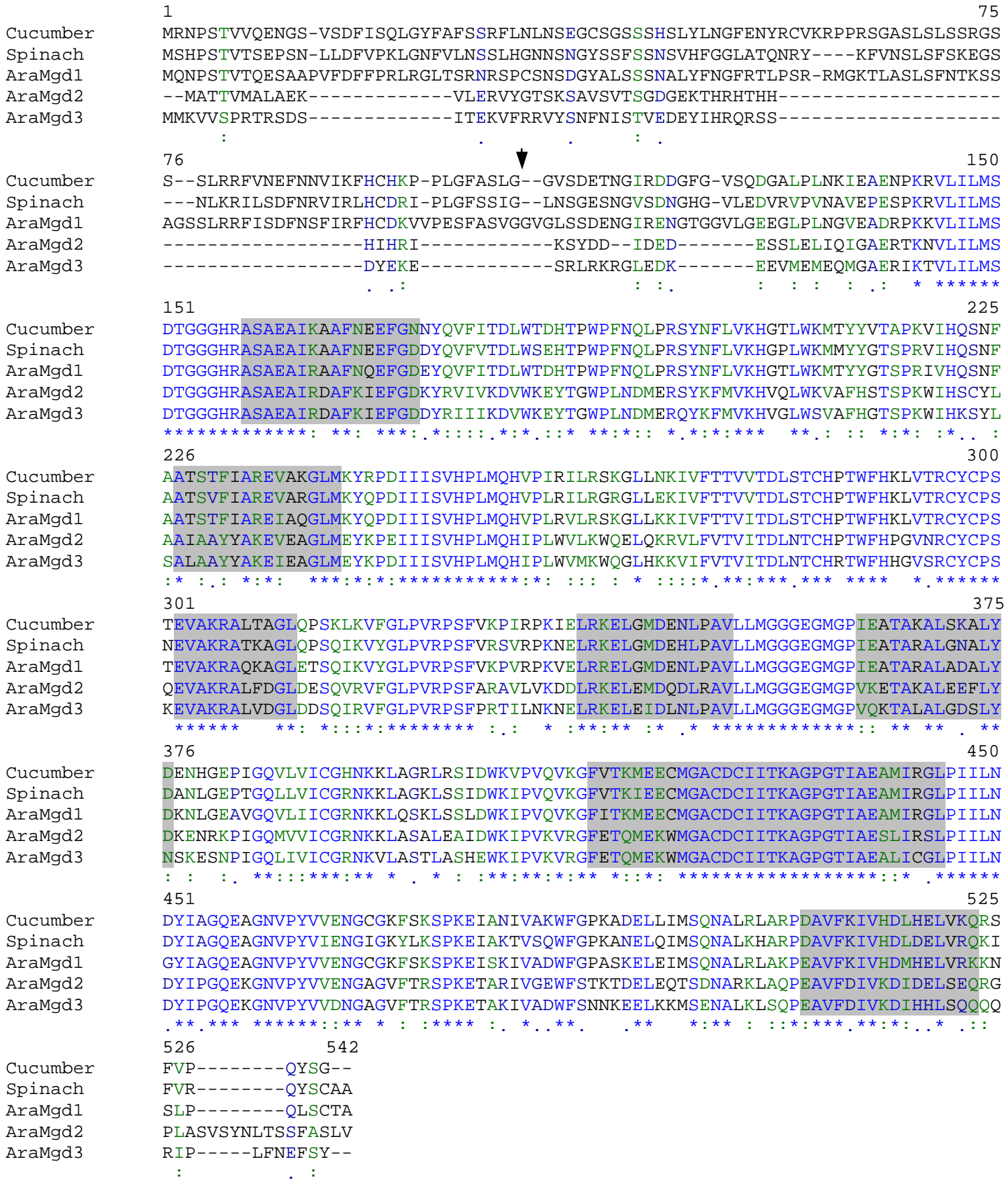

Figure 14. Comparison of amino acid sequences of cDNA encoding MGDG synthases from spinach, cucumber and Arabidopsis. The MGDG synthase sequences from Arabidopsis thaliana, atMGD1 (Ugt81A1), atMGD2 (Acc. No. AJ000331), atMGD3 (BAC F7E22); Cucumis sativa, csMGDA (Acc. No. U62622); and Spinacia oleracea, soMGDA (Acc. No. AJ249607) were compared with CLUSTAL W multiple sequence alignment program (Thompson et al., 1994). The transit peptide cleavage site for the cucumber MGDG synthase upstream of Gly 104 has been determined (Shimojima et al., 1997) and for atMGD1 upstream of Gly 107 was predicted as the cleavage site (Miège et al., 1999), indicated by the arrow. The amino acid numbers starting from the initiation methionine are indicated. Seven putative $\alpha$-helices are boxed in gray. ${ }^{*}$ - single, fully conserved residues; : conservation of strong groups; . - conservation of weak groups. 
complete translocation of proteins to any destination inside chloroplasts. After import chloroplasts were treated with thermolysin to remove proteins that were just bound to the chloroplasts surface. Thermolysin should not penetrate the outer envelope membrane in the concentration used for these experiments. Only the intermembrane space-, inner membrane-, and stroma-localized proteins remain protected after this treatment.

Only about $3 \%$ of pTic22 was processed to its mature form after import, visible as $22 \mathrm{kDa}$ radioactive signal (mTic22), compared to the $28.5 \mathrm{kDa}$ precursor protein (pTic22, Figure 15, lanes 1-3). After posttreatment of chloroplasts with thermolysin (0.5 $\mu \mathrm{g}$ per $1 \mu \mathrm{g}$ chlorophyll), $50 \%$ of mature Tic22, as well as $50 \%$ of precursor form remained intact, protected from the protease action. On the other hand, treatment of the translation product with equivalent amounts of thermolysin led to the complete degradation of Tic22 (Figure 17A, lane 1). The experiments using a higher thermolysin concentrations revealed the same result, with around $50 \%$ protection from thermolysin treatment (compared to the import intensity without pretreatment). These results indicate that the observed forms are indeed imported into chloroplasts. A significant portion of a bound precursor of Tic22 that is protected from thermolysin digestion was probably translocated across the outer envelope membrane, but at this stage not yet processed to the mature form.

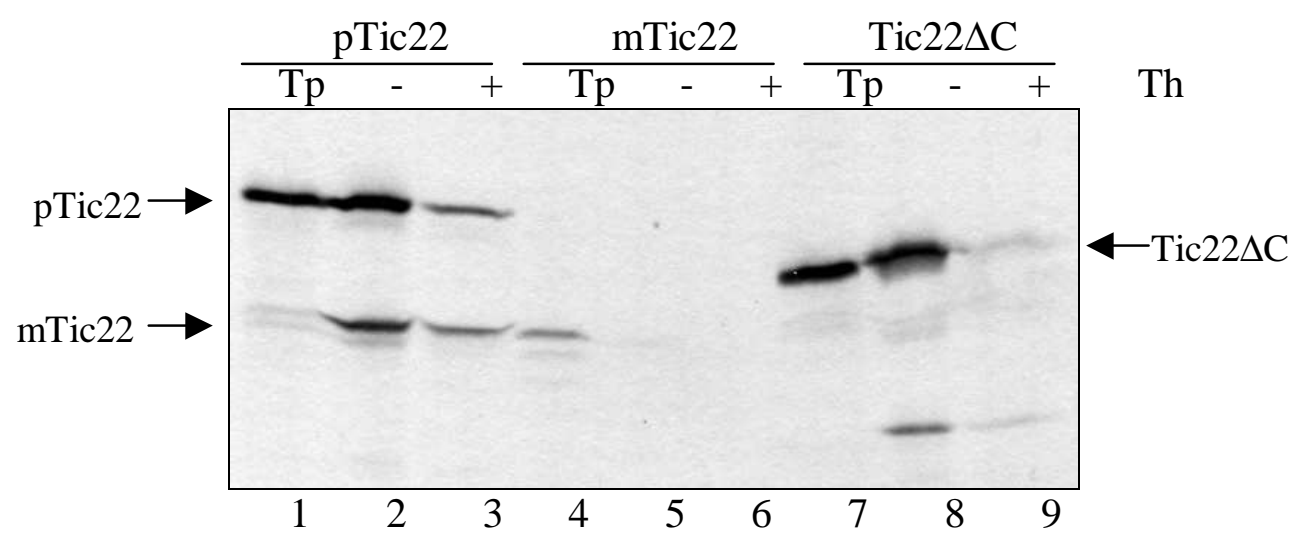

Figure 15. AtTic22 is imported into pea chloroplasts. In vitro synthesized ${ }^{35}$ S-pTic22 (lanes 1-3), ${ }^{35} \mathrm{~S}$-mTic22 (lanes 4-6) and ${ }^{35} \mathrm{~S}$-Tic22 $\Delta \mathrm{C}$ (lanes 7-9) were incubated with isolated intact chloroplasts at $25^{\circ} \mathrm{C}$ for 20 minutes, in a standard import reaction containing $3 \mathrm{mM}$ ATP. After import, samples were reisolated on a Percoll cushion and treated with thermolysin (lanes 3, 6 and 9). Results were analyzed by SDS-PAGE. Lanes 1, 4 and 7 represent $1 / 10$ of the translation products used for the import reactions. The positions of pTic22, mTic 22 and Tic $22 \Delta \mathrm{C}$ are indicated by arrows.

Import of the mature form of Tic22 could not be observed to any extent (Figure 15, lanes 4-6), indicating that the presequence is essential for successful targeting and translocation of Tic22 to the intermembrane space of chloroplasts. The construct lacking $44 \mathrm{C}$-terminal amino 
acids was imported successfully into the intermembrane space (Figure 15, lanes 7-9). These results show that the C-terminus is not important for targeting and final positioning of Tic22, but instead, the complete targeting and localization information is located in its $\mathrm{N}$-terminal targeting presequence.

Import of the radioactively labelled pMGD1 into intact pea chloroplasts resulted in the mature, thermolysin-protected product of around $45 \mathrm{kDa}$. Import was observed already without externally added ATP, but only after addition of $3 \mathrm{mM}$ ATP the mature form was protected from thermolysin digestion (Figure 16A, lane 6 in comparison to lane 4).

A

B

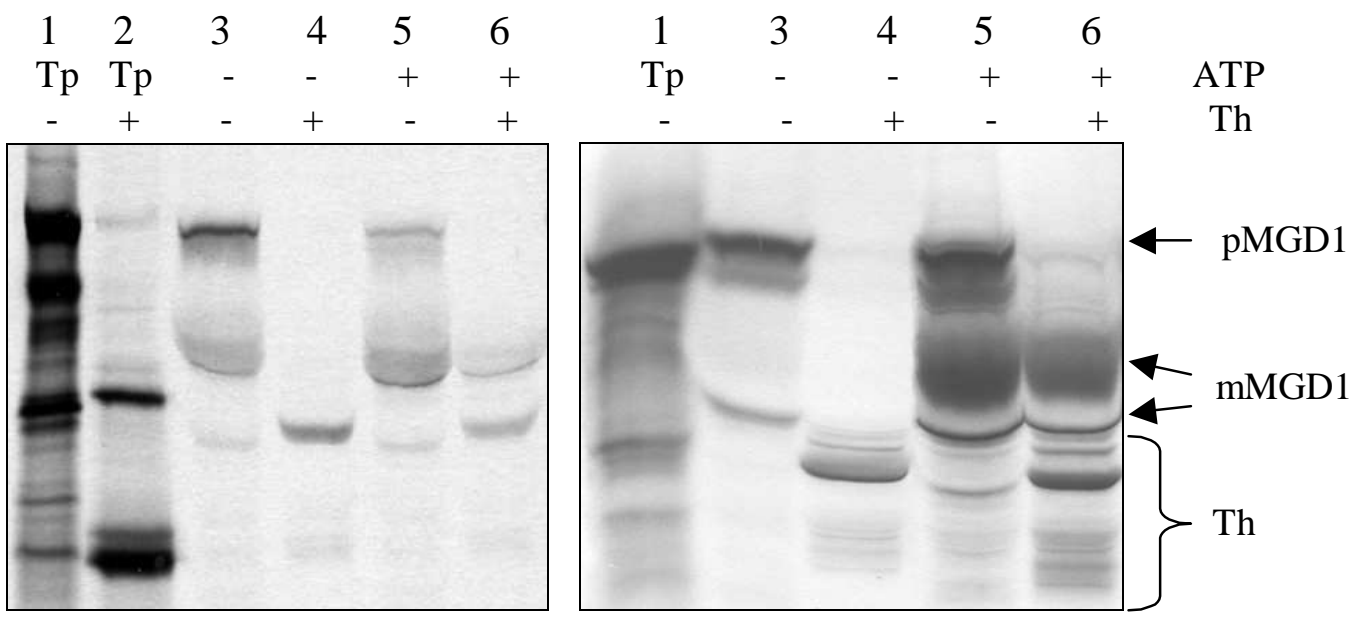

Figure 16. Import of atMGD1 into pea chloroplasts. A. In vitro synthesized ${ }^{35} \mathrm{~S}$-pMGD1 was incubated with isolated intact pea chloroplasts at $25^{\circ} \mathrm{C}$ for 20 minutes, in a standard import reaction. Lane 1 represents $1 / 10$ of the the translation product used for the import. In lane 2 translation product was treated with thermolysin. Import was performed in the absence (lanes 3, 4) or presence (lanes 5, 6) of $3 \mathrm{mM}$ ATP. After import chloroplasts were reisolated on a Percoll cushion and subjected to the treatment with $0.5 \mu \mathrm{g}$ thermolysin per $\mu \mathrm{g}$ chlorophyll (lanes 4,6 ). Untreated samples are shown in lanes 3 and 5. Results were analyzed by SDS-PAGE. B. Import of pMGD1 performed in the presence of $80 \mathrm{mM} \mathrm{K} \mathrm{KO}_{4}$. Precursor protein (pMGD1), mature form (mMGD1) and typical thermolysin degradation pattern $(\mathrm{Th})$ are indicated.

Thermolysin digestion of the translation product revealed a typical pattern (indicated as Th in Figure 16) that was always present after the thermolysin treatment of chloroplasts after import, as well as after treatment of the translation product. Aiming to improve the low MGD1 import efficiency, the results from Hirsch and Soll (1995) were considered, since they showed that the import of an inner chloroplast envelope protein of $96 \mathrm{kDa}$ is greatly stimulated by addition of potassium phosphate.

Though this method did not improve import of Tic22, it greatly stimulated import of MGD1. Addition of potassium phosphate buffer $(80 \mathrm{mM}, \mathrm{pH} 7.6)$ increased the import efficiency of the previously observed $45 \mathrm{kDa}$ form, as well as its other form that was running 
slightly higher on SDS-PAGE (Figure 16B, lanes 5 and 6). The exact size of the mature forms of MGD1 was hard to determine because of its stretched and smeared shape. The observed bands most likely represent two forms of the same mature MGD1 and both show the same import behaviour. The lower, sharper and thinner, band represents a portion of mMGD1 that is pressed by the running behaviour of the large subunit of RubisCO and the upper, stretched and smeared, one was considered to indicate a portion of the mature MGD1 that is associated with lipids. $80 \mathrm{mM} \mathrm{KPi}$ buffer was used in all subsequent import experiments with MGD1.

Import of MGD1 into spinach chloroplasts was performed as well, because Spinacea oleracea as a 16:3 plant is expected to share more similarity with Arabidopsis thaliana concearning MGD1 import properties and topology than with the 18:3 plant Pisum sativum. Import into intact spinach chloroplasts, performed using the same conditions as for pea, gave the same result as shown in Figure 16. All subsequent import experiments have been performed in pea, because of the established conditions for pea growth and methods for intact chloroplasts and envelope isolation in our laboratory that could provide qualitatively and quantitatively uniform results.

\subsubsection{Energy dependence of Tic22 and MGD1 import}

Binding and spontaneous insertion into the chloroplast outer envelope membrane are the only steps of the protein import pathway that do not require energy in the form of ATP. For translocation of a protein across the outer envelope to the intermembrane space, or to the inner envelope and into the stroma, $50 \mu \mathrm{M}$ to $1 \mathrm{mM}$ ATP are required. The effect of an increase of externally added ATP on the import of Tic22 and MGD1 was tested in this study. Protein precursors were synthesized in reticulocyte lysate, a system that also contains an ATP regenerating system. This ATP can be depleted from translation products using gel filtration, apyrase treatment, hexokinase assay or combination thereof (as described in section 5.5.1). Intact pea plants were kept in the dark over night prior to chloroplast isolation and isolated chloroplasts were kept in the dark for at least 30 minutes prior to import. These incubations minimized the production of internal nucleoside triphosphates inside the chloroplasts by photophosphorylation, as well as carbohydrate metabolism. The import reaction was incubated in the dark for the same reason. Consequently, only the externally added ATP was supposed to influence the import rates of proteins tested. As a control for the energy state of chloroplasts during the import reaction, binding and import of pSSU was monitored. 
A

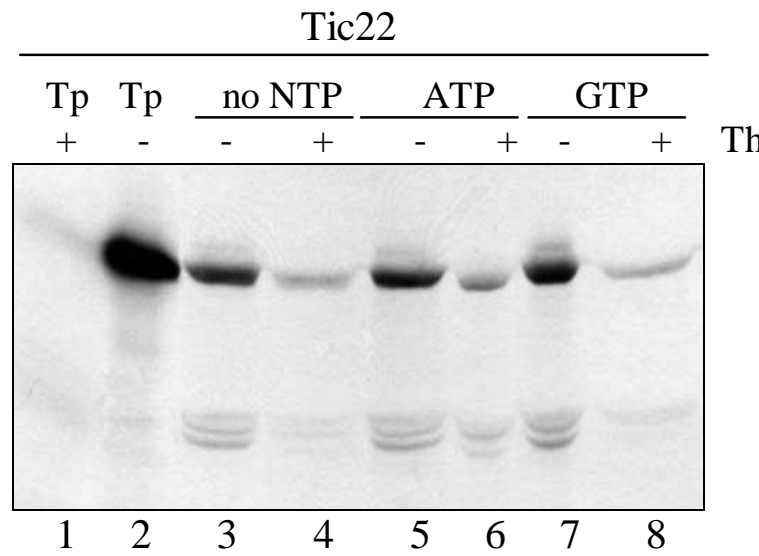

B

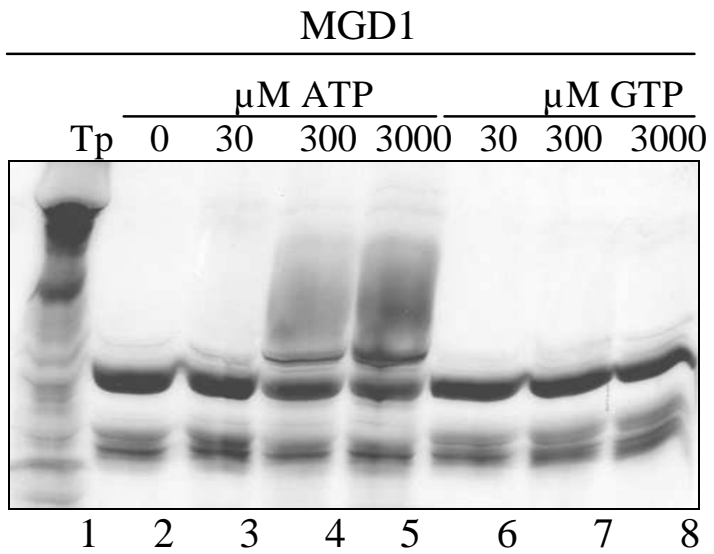

C

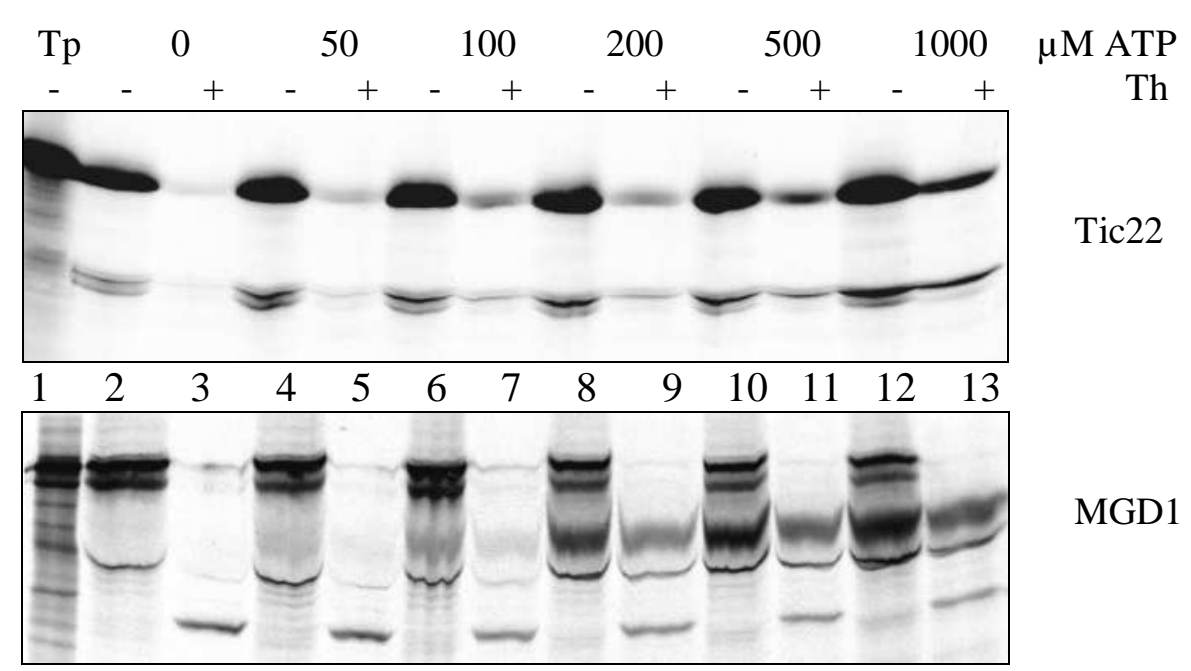

Figure 17. ATP-dependence of Tic22 and MGD1 import. Import into intact pea chloroplasts was performed by incubating in vitro synthesized ${ }^{35}$ S-pTic22 or ${ }^{35}$ S-pMGD1 with chloroplasts corresponding to $20 \mu \mathrm{g}$ chlorophyll, at $25^{\circ} \mathrm{C}$ in a standard import reaction in the dark. Results were analyzed by SDS-PAGE. Tp represents $1 / 10$ of the translation product used for import. A. The import of ${ }^{35} \mathrm{~S}$-pTic22 translation product was performed in the presence of $3 \mathrm{mM}$ ATP (lanes 5 and 6), $3 \mathrm{mM}$ GTP (lanes 7 and 8) or without NTP (lanes 3 and 4). Samples in lanes 1, 4, 6 and 8 were treated with thermolysin after import. B. ${ }^{35} \mathrm{~S}$-pMGD1 translation product was imported in the presence of increasing concentrations of ATP (lanes 3-5) or GTP (lanes 6-8). All samples were treated with thermolysin after the import reaction. C. For import, increasing concentrations of ATP, from 0 to 1000 $\mu \mathrm{M}$ (lanes 2-13) were used. After import chloroplasts were reisolated on a Percoll cushion and incubated in the presence (lanes $3,5,7,9,11,13$ ) or absence (lanes $2,4,6,8,10,12$ ) of $0.5 \mu \mathrm{g}$ thermolysin per $\mu \mathrm{g}$ chlorophyll.

Radioactively labelled pTic22 and pMGD1 were imported into intact pea chloroplasts corresponding to $20 \mu \mathrm{g}$ chlorophyll, for 20 minutes at $25^{\circ} \mathrm{C}$. Both Tic22 and MGD1 imported into chloroplasts already at very low ATP concentrations, but the processed forms were almost completely degraded by thermolysin already at the early time points (Figure 17C, lanes 2-5). Import in the presence of GTP, as the only NTP added to the import reaction, was not observed for any of the proteins (Figure 17A, lanes 7-8 and B, lanes 6-8). Posttreatment 
with thermolysin showed that a portion of the protease resistant mature form increases with increasing ATP concentrations. After import of Tic22 into pea chloroplasts, two bands of similar size could be distinguished. Since the lower one was removed by thermolysin treatment, only the upper one, which was changing its intensity in response to changed import conditions, was considered as the mature form of Tic22.

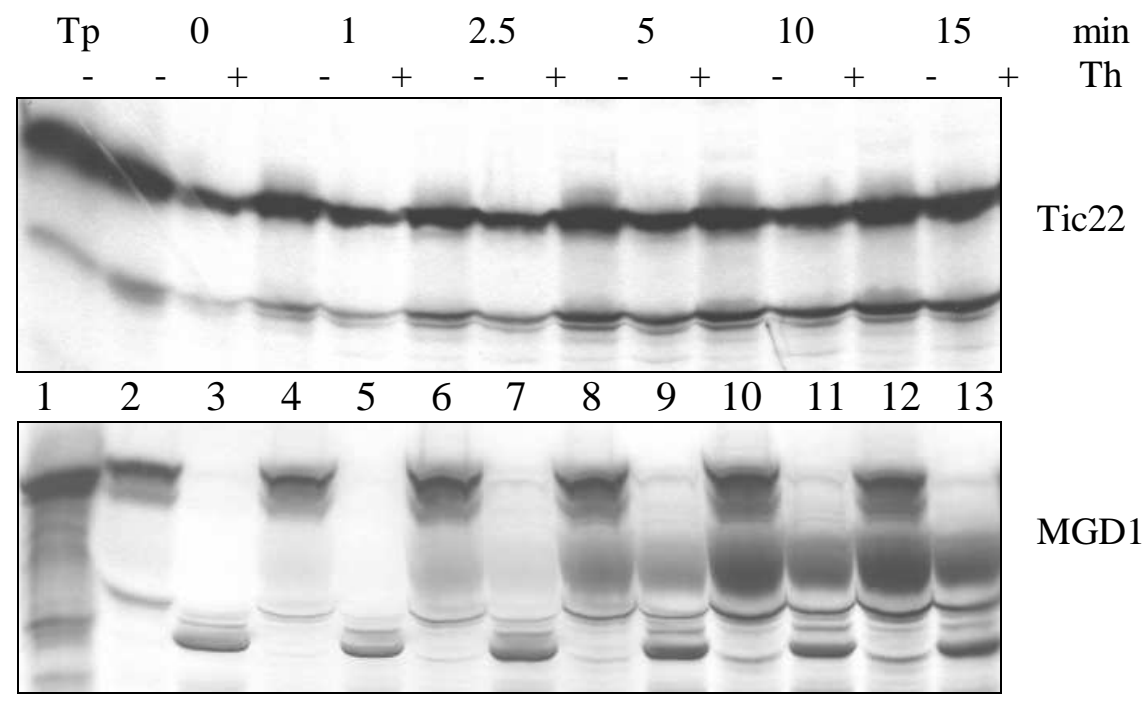

Figure 18. Time dependence of Tic22 and MGD1 import. Import into intact pea chloroplasts was performed using the standard protocol, by incubating in vitro synthesized ${ }^{35} \mathrm{~S}-\mathrm{pT}$ Tic22 and ${ }^{35} \mathrm{~S}$-pMGD1 with chloroplasts corresponding to $20 \mu \mathrm{g}$ chlorophyll at $25^{\circ} \mathrm{C}$ in the presence of $3 \mathrm{mM}$ ATP. The time of import reaction varied from 0 to 15 minutes (lanes 2-13). After import, chloroplasts were reisolated on a Percoll cushion and incubated in the presence (lanes 3, 5, 7, 9, 11, 13) or the absence (lanes 2, 4, $6,8,10,12)$ of $0.5 \mu \mathrm{g}$ thermolysin per $\mu \mathrm{g}$ chlorophyll. Results were analyzed by SDS-PAGE. Lane 1 represents $1 / 10$ of the translation product used for import.

Both forms observed for MGD1 showed the same ATP dependent import behaviour. After addition of 100-200 $\mu \mathrm{M}$ ATP, 50\% of import was accomplished and $500 \mu \mathrm{M}$ ATP was required for achieving the maximal import rate (around 3\% for Tic22 and up to $20 \%$ for MGD1). Import experiments performed for various times using a standard import reaction in the presence of $3 \mathrm{mM}$ ATP showed similar behaviour for both proteins (Figure 18). Sensitivity to protease treatment decreased with time for both Tic22 and MGD1. 
A

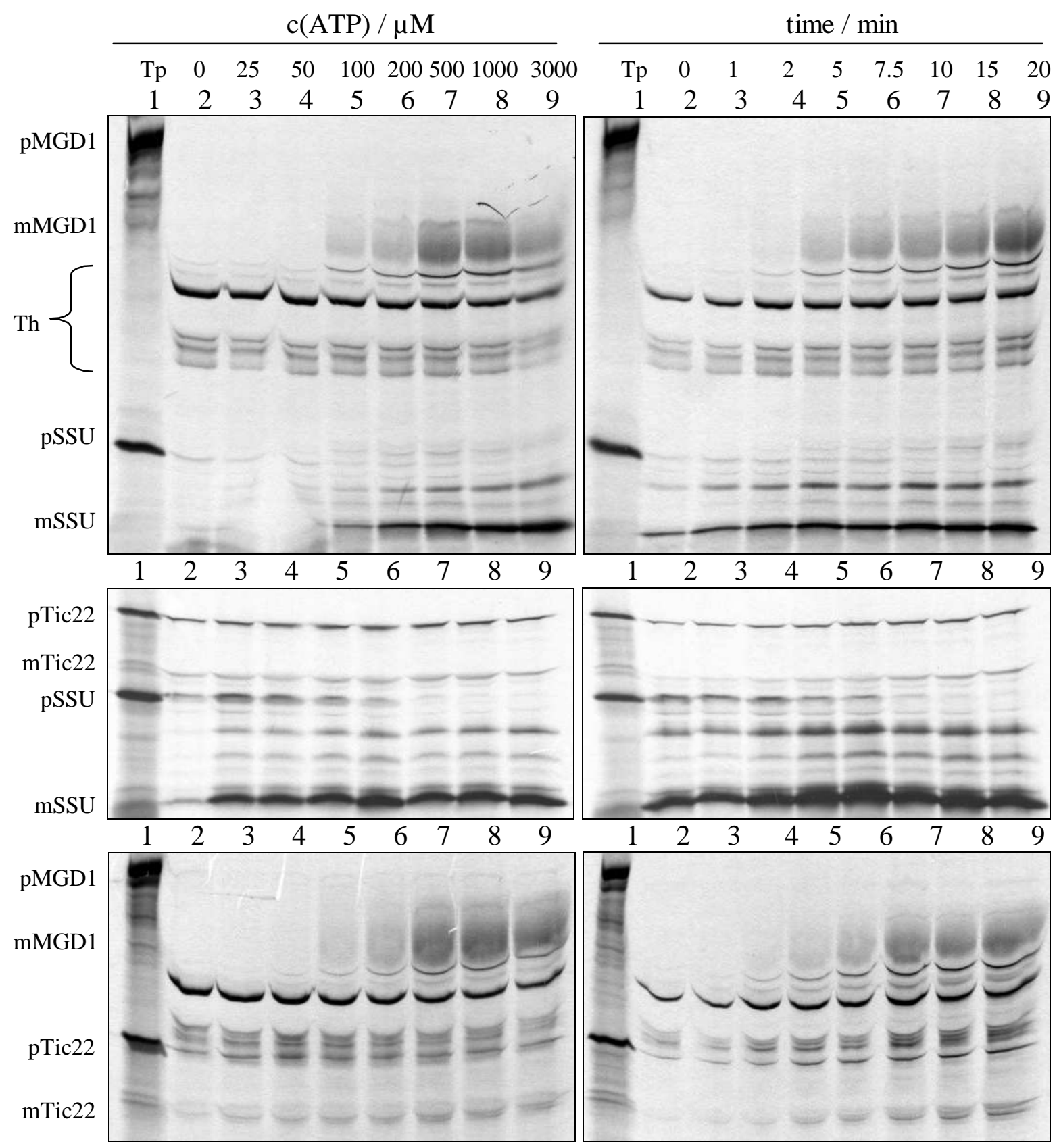

B
C

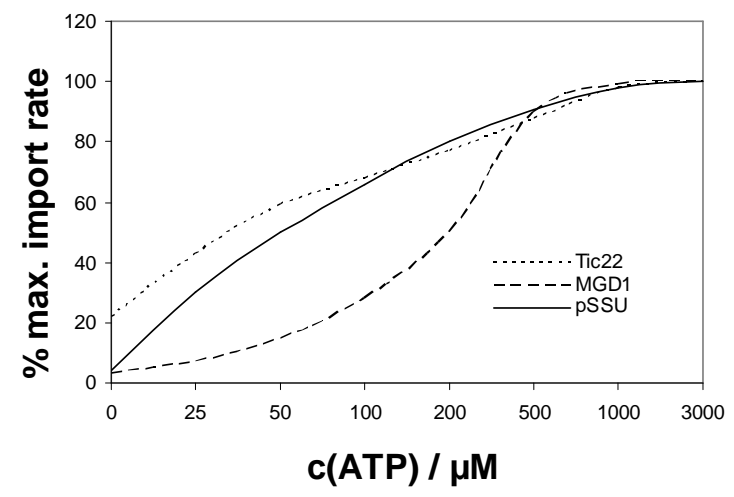


Figure 19. Comparison of ATP- and time-demands for import of Tic22, MGD1 and SSU. Import into intact pea chloroplasts was performed using the standard protocol, by incubating in vitro synthesized ${ }^{35} \mathrm{~S}$-pTic22 and ${ }^{35} \mathrm{~S}$-pMGD1 with chloroplasts corresponding to $20 \mu \mathrm{g}$ chlorophyll, at $25^{\circ} \mathrm{C}$. Parallel imports combining ${ }^{35} \mathrm{~S}$-pMGD1 and ${ }^{35} \mathrm{~S}$-pSSU, ${ }^{35} \mathrm{~S}-\mathrm{pTic} 22$ and ${ }^{35} \mathrm{~S}$-pSSU and ${ }^{35} \mathrm{~S}$-pTic22 and ${ }^{35} \mathrm{~S}$-pMGD1, in the same reaction were performed. After import, chloroplasts were reisolated on a Percoll cushion and all samples were treated with thermolysin. Results were analyzed by SDS-PAGE. Lane 1 represents 1/10 of the translation product used for import. A. ATP-scale import into intact pea chloroplasts was performed using increasing concentrations of ATP from 0 to $3000 \mu \mathrm{M}$ (lanes 2-9) for 15 minutes at $25^{\circ} \mathrm{C}$. B. Time-scale import into intact pea chloroplasts was performed using increasing time from 0 to 20 minutes and $3 \mathrm{mM}$ ATP at $25^{\circ} \mathrm{C}$ (lanes 2-9). C. ATP- and timedependent import reactions from five independent experiments were quantified and results presented graphically.

The presence of high concentrations of ATP was sufficient for partial import of Tic22 even on ice (Figure 18, lanes 2 and 3), while MGD1 started to import efficiently only after around 2.5 minutes (Figure 18, lanes 6 and 7). After 10 minutes the import of both Tic22 and MGD1 was nearly completed (Figure 18, compare lanes 8 and 9 for Tic22 and 10 and 11 for MGD1).

To gain a better insight into the correlation between Tic22 and MGD1, imports under the influence of modification of ATP concentrations and time-scale were conducted, and to get a better comparison of import needs between those two intermembrane space proteins, parallel imports combining ${ }^{35}$ S-pTic22 and ${ }^{35}$ S-pMGD1, ${ }^{35}$ S-pTic22 and ${ }^{35}$ S-pSSU and ${ }^{35} \mathrm{~S}$-pMGD1 and ${ }^{35} \mathrm{~S}$-pSSU in the same reaction were performed (Figure 19). Results obtained by these experiments suggest that Tic 22 imports into chloroplasts relatively quickly. Around $20 \%$ of total Tic22 imported, translocated without addition of external ATP (Figure 19A, lane 2) and the final maximal import rate was reached at 0.5 to $1 \mathrm{mM}$ ATP (Figure 19A, lanes 7 and 8). The ATP-dependent import curve of Tic22 follows the one of the stromal protein pSSU, indicating that its import rate progresses in response to the increase of ATP concentration (Figure 19C). These results point to the conclusion that binding of Tic22 to chloroplasts does not require ATP. Also, a smaller portion of the preprotein is imported into chloroplasts under limited ATP conditions, but increase of the ATP concentration enhances its import and enables its complete translocation. In the presence of $3 \mathrm{mM}$ ATP, already after 5 minutes at $25^{\circ} \mathrm{C}, 80 \%$ of maximal import rate for pSSU was reached (Figure 19B, lane 5, Figure 19C). In contrast, Tic22 and MGD1 require 10 to 15 minutes and $3 \mathrm{mM}$ ATP to achieve the same import rate (Figure 19B, lanes 7 and 8, Figure 19C). More than $100 \mu \mathrm{M}$ ATP was required for efficient import of MGD1, but its maximal import rate was achieved at 0.5 to $1 \mathrm{mM}$ ATP, the same concentrations as observed for Tic22.

At this point it could be concluded that the import of MGD1 is dependent on and highly stimulated by ATP, while import of Tic22 is dependent on ATP hydrolysis to a lesser extent. 


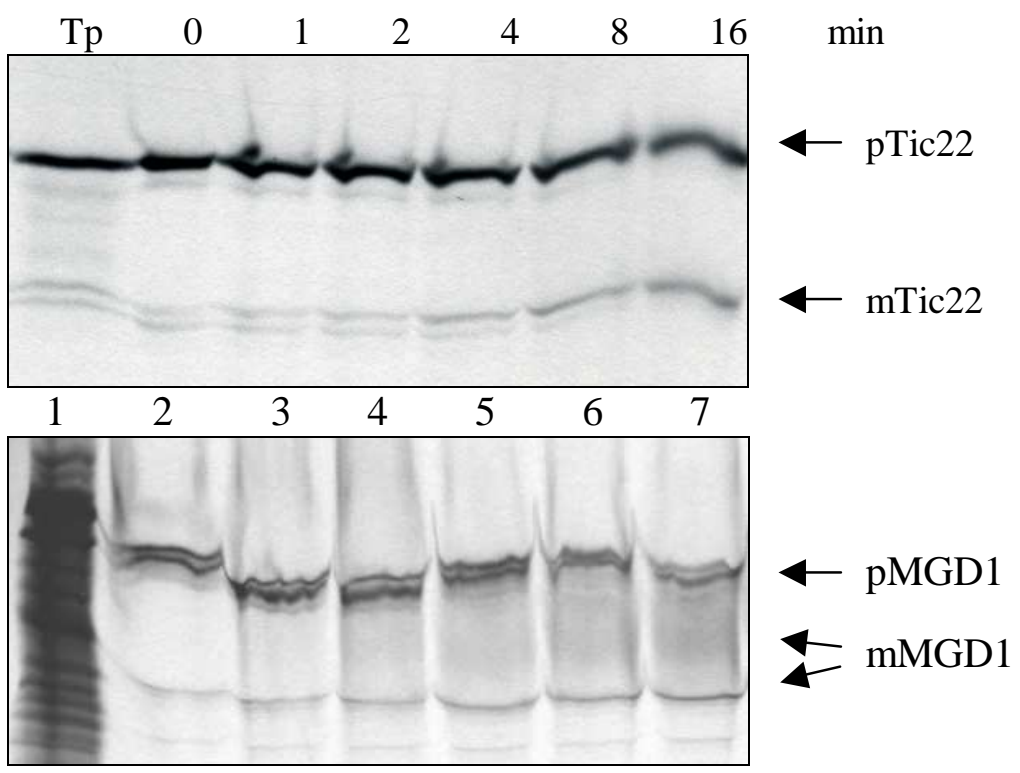

Figure 20. Pulse and chase of Tic22 and MGD1. Binding to intact pea chloroplasts was performed by incubating the in vitro synthesized ${ }^{35} \mathrm{~S}$-pTic22 and ${ }^{35} \mathrm{~S}$-pMGD1 with chloroplasts corresponding to $20 \mu \mathrm{g}$ chlorophyll at $4^{\circ} \mathrm{C}$ in a standard import reaction lacking ATP. In the reaction mixtures containing MGD1 $80 \mathrm{mM} \mathrm{K}_{\mathrm{i}} \mathrm{PO}_{4}$ was added. After 2 minutes of binding, chloroplasts were reisolated and resuspended in the import mix containing $3 \mathrm{mM} \mathrm{ATP}$, and import reaction was performed for different times at $25^{\circ} \mathrm{C}$ (lanes 3-7). Reaction was stopped by addition of Laemmli buffer and results were analyzed by SDS-PAGE. The control reaction was performed on ice (lane 2). Lane 1 indicates $1 / 10$ of the translation product used for the import reaction.

Different needs for energy of those two proteins suggest that they might use different pathways for their import into chloroplasts.

Using a pulse-chase experiment (Figure 20), import behaviour of precursor proteins and arising of mature forms can be followed. Radioactively labelled precursors were incubated with intact pea chloroplast in a standard import reaction, using $20 \mu \mathrm{g}$ chlorophyll. The reaction was incubated for two minutes on ice, without addition of ATP. Those conditions allowed binding of precursor proteins to the protein import receptors on the chloroplast surface. After 2 minutes the chloroplasts were pelleted and reisolated in a fresh import medium containing $3 \mathrm{mM}$ ATP. The unbound precursors were removed by this step. After 1, 2, 4, 8, and 16 minutes import reactions were stopped by addition of Laemmli buffer and samples were analyzed by SDS-PAGE. After 4 minutes a significant increase in import rates of both Tic22 and MGD1 proteins could be observed (Figure 20, lane 5). The maximal import rate was achieved between 8 and 16 minutes (Figure 20, lanes 6 and 7). 


\subsubsection{Processing of the precursor proteins pTic22 and pMGD1}

To address the question whether Tic22 and MGD1 use the same pathway for the translocation into chloroplast, determination of where the processing of precursor proteins takes place was attempted. The stromal processing peptidase is the only stroma-localized enzyme known to cleave signal peptides of preproteins in the course of the general import pathway (Abad et al., 1989, Richter and Lamppa, 1999). To investigate whether this enzyme is responsible for the processing of pTic22 and pMGD1, chloroplast stroma was isolated, and a stromal processing assay performed as described in the section 5.8. It was observed that pMGD1, as well as the control protein $\mathrm{pSSU}$, was processed to its mature form in the presence of the stromal extract (Figure 21, indicated by arrows). After processing of pMGD1, a band of around $45 \mathrm{kDa}$ could be observed (Figure 21, lanes 5 and 6). When chloroplasts were added to the sample to provide a lipid environment, the same pattern was observed as during the import of MGD1 (Figure 21B, lanes 3 and 4).

A

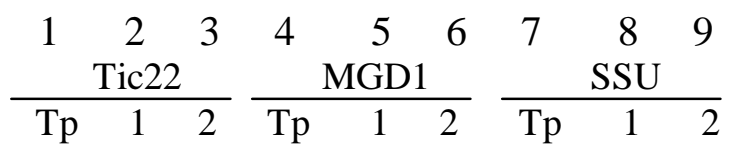

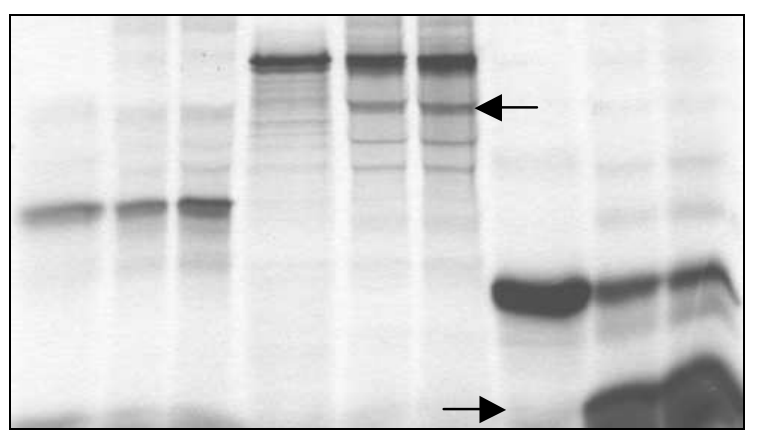

B

\begin{tabular}{ccrc}
1 & 2 & 3 & 4 \\
& MGD1 & \\
\hline Tp & SP & SP & SP \\
- & - & + & +
\end{tabular}

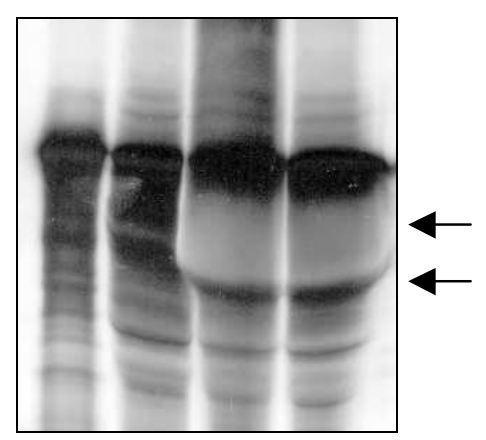

Figure 21. pMGD1, but not pTic22, is processed by the stromal processing peptidase. A. Stroma was isolated from pea chloroplasts and incubated with radioactively labelled translation products ${ }^{35} \mathrm{~S}$-pTic22, ${ }^{35} \mathrm{~S}$-pMGD1 and ${ }^{35} \mathrm{~S}$-pSSU for 90 minutes at $26^{\circ} \mathrm{C}$. Reactions were stopped by addition of Laemmli buffer and samples were analyzed by SDS-PAGE. Lanes 1, 4, and 7 represent $2 \mu 1$ of the corresponding translation products, and lanes 2-3, 5-6 and 8-9 represent 2 and $3 \mu 1$ of the processed translation product. Mature forms of MGD1 and SSU, appearing after processing by a stromal processing assay, are indicated by arrows. B. After the stromal processing of ${ }^{35} \mathrm{~S}$-pMGD1, $5 \mu \mathrm{g}$ of chloroplasts were added to the reaction, prior to the addition of Laemmli buffer. The lipid-associated form of MGD1 could be observed under those conditions.

The failure of the stromal extract to process the pTic22 (Figure 21A, lanes 2 and 3) led to the conclusion that Tic22 and MGD1 might use distinct pathways at a later stage of their import into the intermembrane space. In this scheme, MGD1 could transverse the Tic-machinery and 
expose its $\mathrm{N}$-terminal presequence to the stroma, where it is being cleaved and the mature protein is subsequently pulled back to its final location in the intermembrane space.

\subsection{3 pTic22 and pMGD1 depend on proteinaceous components on the chloroplast surface for their import}

Import of precursor proteins into chloroplasts requires protease-sensitive components on the outer envelope membrane. Thermolysin pretreatment can remove protein receptors on the chloroplast surface by proteolytic degradation. After this treatment, import of precursor proteins that require intact proteinaceous components should be inhibited. Intact chloroplasts were treated with $1 \mathrm{mg}$ thermolysin per $1 \mathrm{mg}$ chlorophyll for 30 minutes on ice in the dark. After treatment, intact chloroplasts were reisolated on a Percoll gradient in the presence of $5 \mathrm{mM}$ EDTA and used for import reactions. In parallel, non-treated chloroplasts were used in control reactions. The radioactively labelled pTic22, pMGD1 and the control protein pSSU, which import is known to be dependent on thermolysin sensitive components on the chloroplast surface, were incubated with chloroplasts corresponding to $15 \mu \mathrm{g}$ chlorophyll at $25^{\circ} \mathrm{C}$ for $10-12$ minutes for pTic22 and pMGD1, and 5 minutes for pSSU. The experiments resulted in a significant effect of the thermolysin pretreatment on import of all tested proteins (Figure 22). The import yield of pSSU after thermolysin pretreatment was reduced to $30 \%$, of pTic22 to $40 \%$ and of pMGD1 to $50 \%$ (Figure 22B and C), indicating that all these proteins need protein receptors on the chloroplast surface for the initial step of their import into chloroplasts.

\subsection{4 pTic22 and pMGD1 compete with pOE33 for import into isolated pea chloroplasts}

Import competition experiments were performed by binding of a single concentration of labelled translation product in the presence of various concentrations of unlabelled competitor protein. For the unlabelled competitor protein, like the oxygen-evolving complex precursor protein pOE33 used in this study, the import pathway is known. pOE33 uses the general import pathway through Toc- and Tic-complexes for its import into chloroplasts. Therefore, different concentrations of overexpressed and freshly purified pOE33 were added to a standard import reaction. 
A

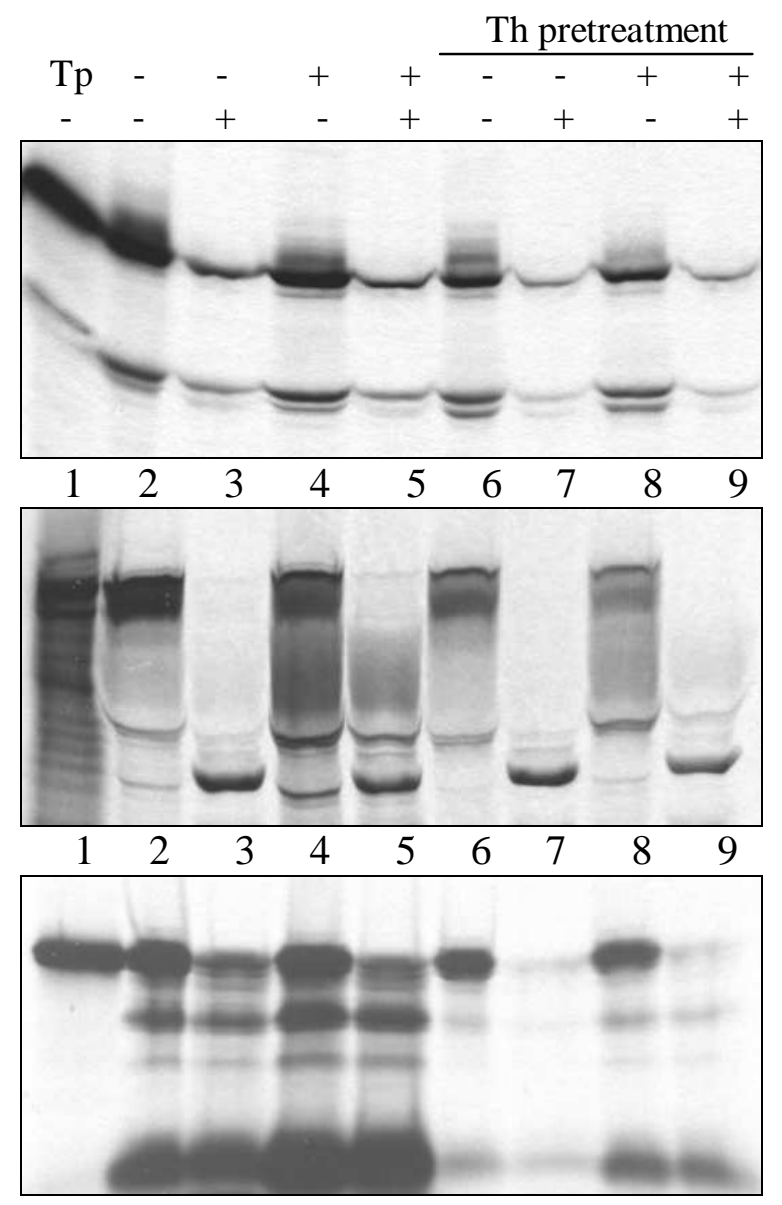

B

ATP

Th

Tic22
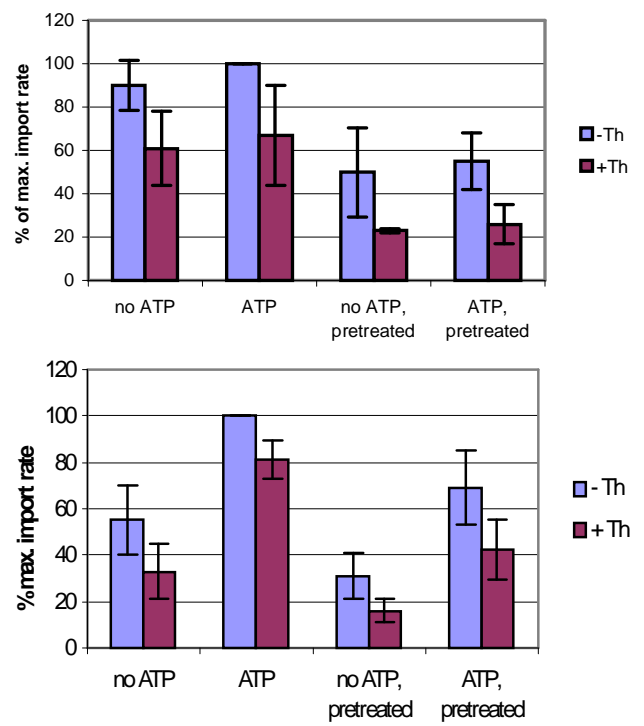

SSU

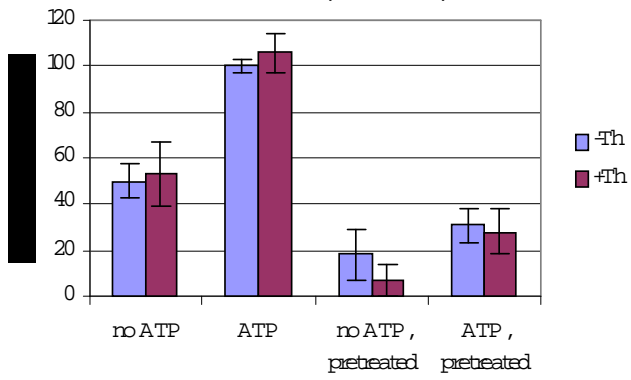

C

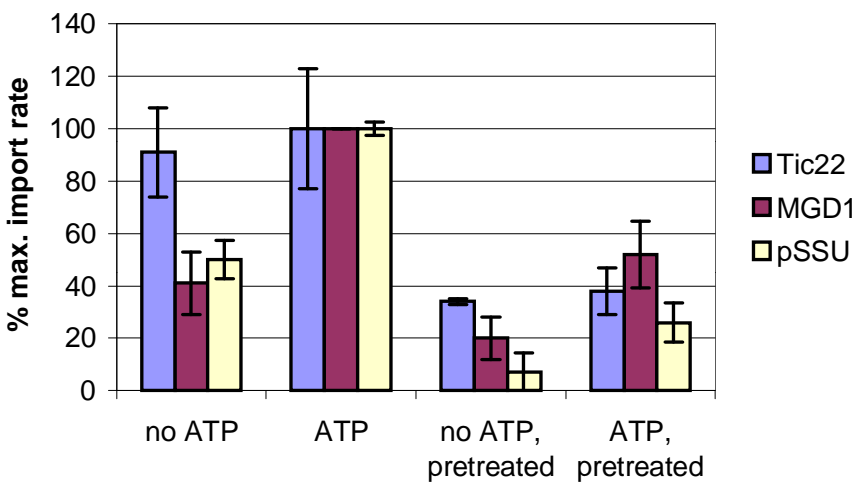

Figure 22. Import of pTic22 and pMGD1 is inhibited by thermolysin pretreatment of chloroplasts. A. For import, intact chloroplasts were used that were either pretreated (lanes 6-9) or not treated (lanes 2-5) with $1 \mathrm{mg}$ thermolysin per $1 \mathrm{mg}$ chlorophyll. Import of ${ }^{35} \mathrm{~S}-\mathrm{pT}$ Tic22, ${ }^{35} \mathrm{~S}$-pMGD1 and ${ }^{35} \mathrm{~S}$-pSSU into intact pea chloroplasts corresponding to $15 \mu \mathrm{g}$ chlorophyll was performed for 15 minutes at $25^{\circ} \mathrm{C}$ for pTic22 and pMGD1, and 5 minutes for pSSU. After import chloroplasts were either subjected to thermolysin posttreatment (lanes 3, 5, 7, 9) or not (lanes 2, 4, 6, 8). Lane 1 represents $1 / 10$ of the translation product used for import. B. Graphical presentation of the influence of thermolysin pretreatment of chloroplasts on import of pTic22, pMGD1 and pSSU, derived by 2D densitometry evaluation (AIDA image analyser) of five independently performed experiments for each protein. C. Direct comparison of the effect of thermolysin pretreatment on import of pTic22, pMGD1 and pSSU, based on the results presented in $\mathrm{B}$. 
A
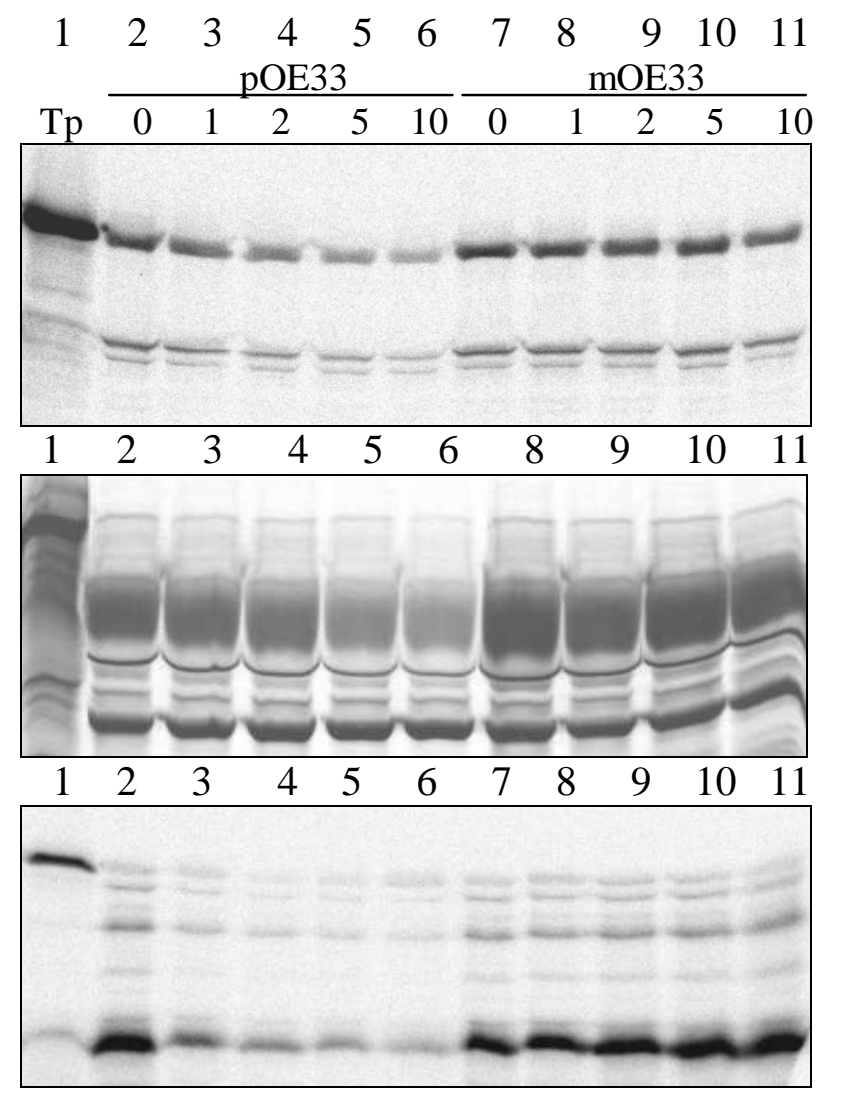

C
B

$\mu \mathrm{M}$

pTic22

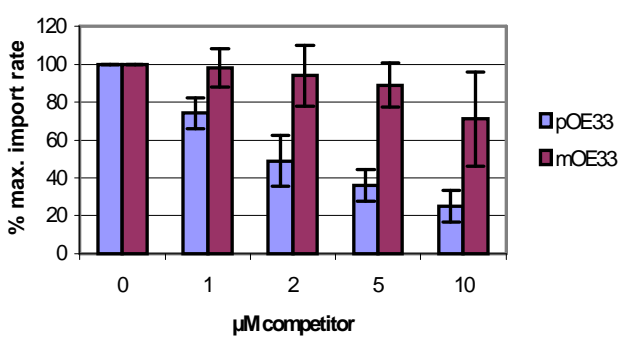

pMGD1

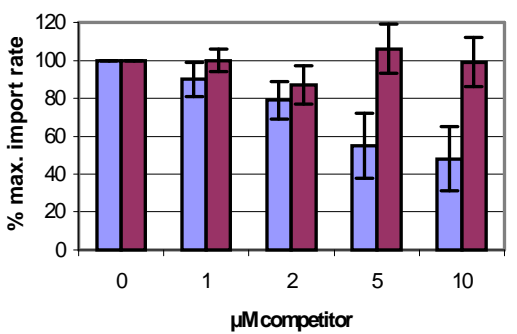

口pOE33

DMOE33

pSSU

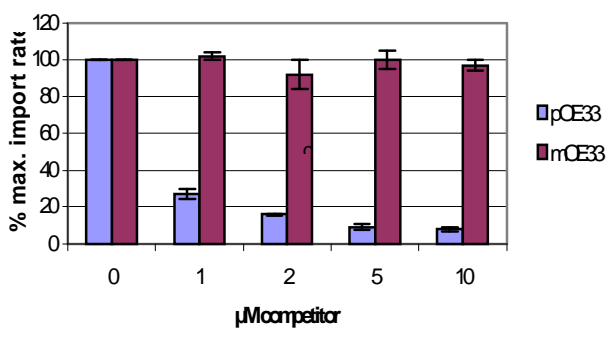

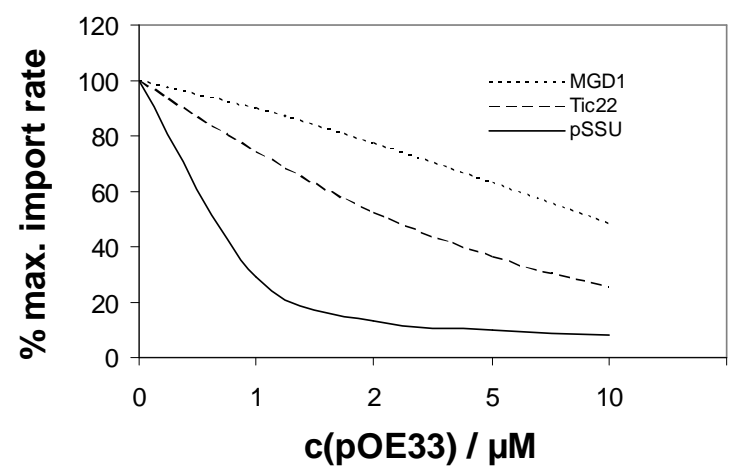

Figure 23. pTic22 and pMGD1 compete with pOE33 for import into chloroplasts. A. Increasing concentrations of overexpressed protein pOE33 (lanes 2-6) or its mature form mOE33 (lanes 7-10) were added into the import mix prior to import of ${ }^{35} \mathrm{~S}$-pTic22, ${ }^{35} \mathrm{~S}$-pMGD1 and ${ }^{35} \mathrm{~S}$-pSSU into intact pea chloroplasts corresponding to $15 \mu \mathrm{g}$ chlorophyll. Import reaction was performed for 12 minutes at $25^{\circ} \mathrm{C}$ for pTic22 and pMGD1 and 5 minutes for the pSSU control. After import, chloroplasts were subjected to thermolysin posttreatment. Lane 1 represents $1 / 10$ of the translation product used for import. B. Graphical presentation of the results presented in A, estimated on the basis of five independently performed competition experiments. C. Direct comparison of pOE33 competition effect on import of pTic22, pMGD1 and pSSU, based on the results presented in B. 
Radioactively labelled pTic22, pMGD1 and pSSU translation products were incubated in the presence of increasing concentrations of pOE33 (up to $10 \mu \mathrm{M}$ ). The import reaction lasted 10-12 minutes for pTic22 and pMGD1 and 5 minutes for pSSU.

Chloroplasts were added into the reaction mixture at the very end to avoid non-competitive import reactions of any of the proteins before all components of interest were added. Mature OE33, mOE33, was used as the negative control for this reaction. Because it lacks the presequence it cannot be recognized by the Toc-complex and cannot use the general import pathway. The results show that pOE33 exhibits the strongest effect on the import of pSSU, where application of just $1 \mu \mathrm{M}$ pOE33 caused the decrease in import yield of about $75 \%$ (Figure 23). $2 \mu \mathrm{M}$ of pOE33 inhibited import of pTic22 to 50\%, and after the application of $10 \mu \mathrm{M}$ inhibitor only around $25 \%$ residual import remained. For pMGD1 this effect was slightly different. The maximal inhibition observed after application of $10 \mu \mathrm{M}$ pOE33 was $50 \%$ (Figure 23). Control experiments using mOE33 as the competitor showed that it had no effect on the import of any of the tested preproteins. These results suggest that both pTic22 and pMGD1 competed for import with pOE33. Although the import of pMGD1 was competed to a lower extent than of pTic22, it is obvious that both proteins translocate into the intermembrane space through the Toc-translocon at the outer envelope membrane.

\subsubsection{Toc34 $\Delta \mathrm{TM}$ competes with the endogenous Toc34 for binding to pTic22 and pMGD1 prior to import into isolated chloroplasts}

Thermolysin pretreatment showed that pTic22 and pMGD1 require thermolysin sensitive components on the chloroplast surface for their import into chloroplasts. The results obtained by the competition experiments using pOE33 suggested that both proteins import into chloroplasts through the Toc-translocon at the outer envelope. Thus, it is probable that pTic22 and pMGD1 also use Toc34 as the initial receptor protein. The interaction of pTic22 and pMGD1 with Toc34 receptor GTPase was tested by performing binding experiments.

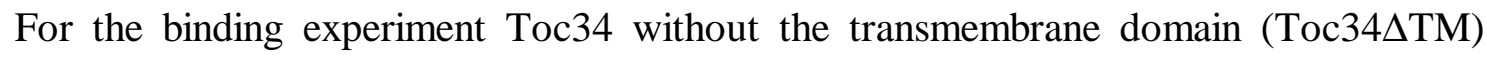
was overexpressed and purified under native conditions (section 5.10.4) and used fresh for further experiments. Purified Toc34 for 10 minutes on ice. Subsequently, ${ }^{35}$ S-pTic22, ${ }^{35}$ S-pMGD1 and ${ }^{35}$ S-pSSU were added and incubation was prolonged for another 10 minutes on ice. After this time, chloroplasts corresponding to $15 \mu \mathrm{g}$ chlorophyll were added and import was performed at $25^{\circ} \mathrm{C}$ for $10-12$ minutes for pTic22 and pMGD1 and 5 minutes for pSSU. 
A
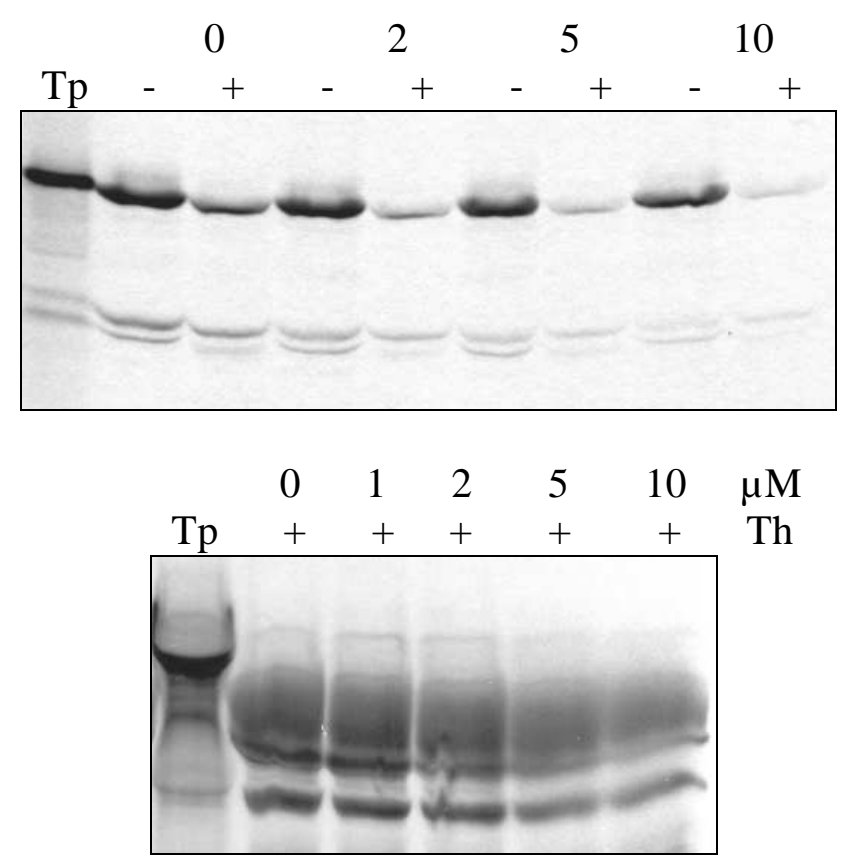

$\mu \mathrm{M}$

$\mathrm{Th}$

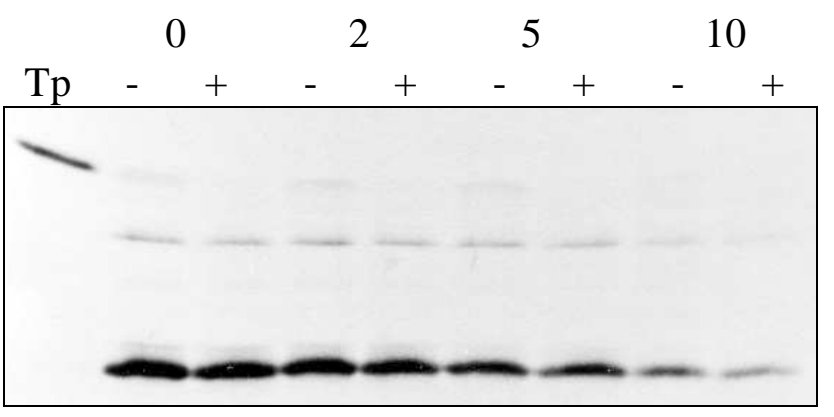

pMGD1

B

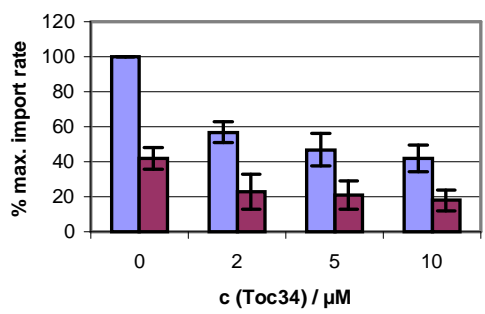

$\square-T h$

口+Th

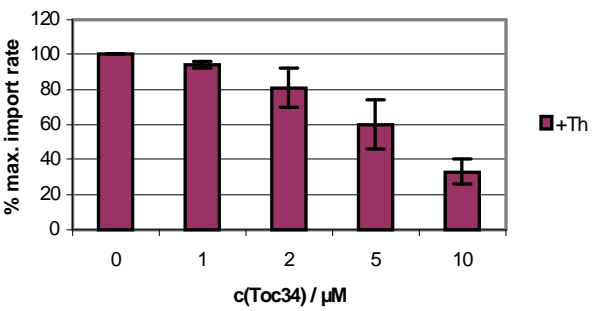

$\mu \mathrm{M}$

Th

pSSU

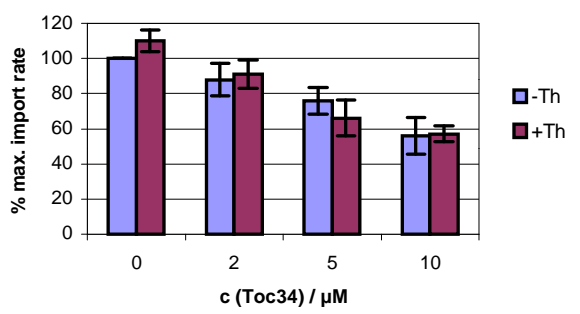

Figure 24. Import of pTic22 is inhibited by the soluble domain of the receptor protein Toc34. Increasing concentrations $(0-10 \mu \mathrm{M})$ of overexpressed soluble receptor GTPase Toc $34 \Delta \mathrm{TM}$, as well as $3 \mathrm{mM}$ ATP and $3 \mathrm{mM}$ GTP, were added to the import mix prior to import of ${ }^{35} \mathrm{~S}$-pTic22, ${ }^{35} \mathrm{~S}$-pMGD1 and ${ }^{35} \mathrm{~S}$-pSSU into intact pea chloroplasts corresponding to $15 \mu \mathrm{g}$ chlorophyll. Import reaction was performed for 12 minutes at $25^{\circ} \mathrm{C}$ for pTic22 and pMGD1 and 5 minutes for the pSSU control. After import, chloroplasts were either subjected to thermolysin posttreatment $(+\mathrm{Th})$ or not $(-\mathrm{Th})$. $\mathrm{Tp}$ indicates $1 / 10$ of the translation product used for import. B. Graphical presentation of the results presented in A, estimated on the basis of five independently performed competition experiments for each preprotein.

With increasing concentrations of Toc $34 \Delta \mathrm{TM}$ a decrease in import rate was observed for all tested proteins (Figure 24). This finding indicates that the soluble Toc34 $\Delta \mathrm{TM}$ interacts with precursor proteins prior to import and less of "free" precursor proteins remain to be imported into chloroplasts. The effect observed was the strongest for pTic22, indicating its strong interaction with Toc34. Interaction of MGD1 and Toc34 $\Delta \mathrm{TM}$ could be compared to the interaction with pSSU (Figure 24B), although the highest competitor concentration used $(10 \mu \mathrm{M})$ caused stronger inhibition effect on import of MGD1 ( 35\% residual import compared to $\sim 60 \%$ for $\mathrm{pSSU})$. 
A
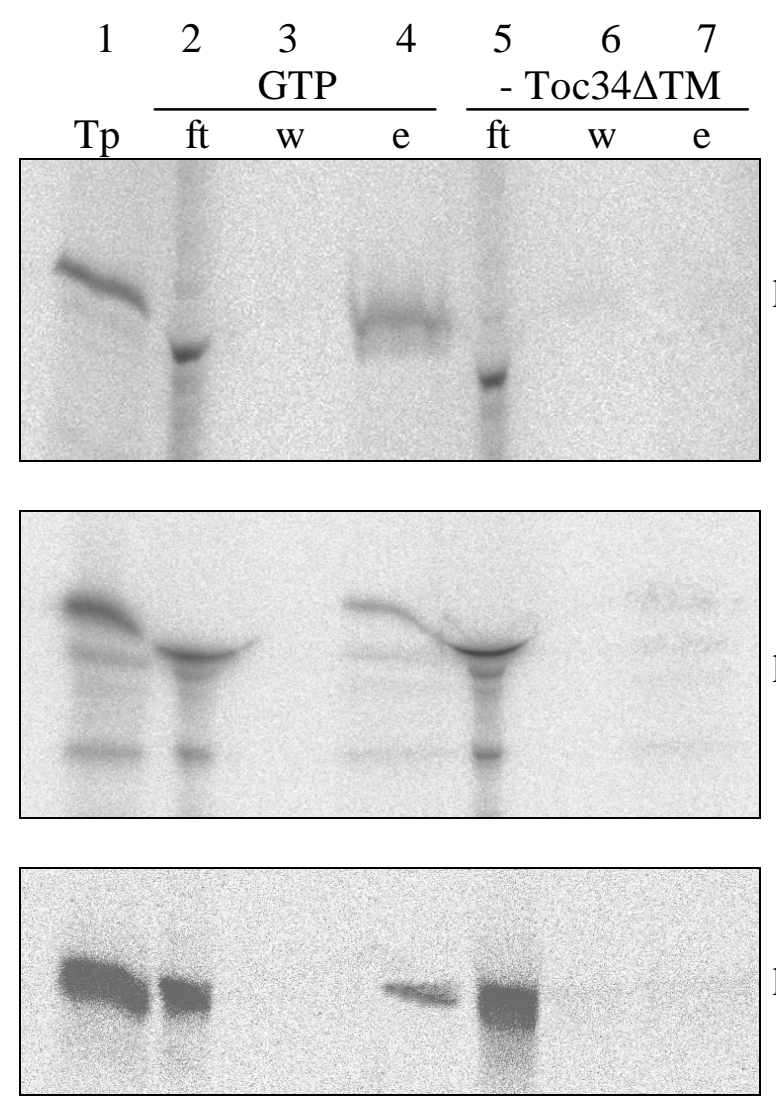

B

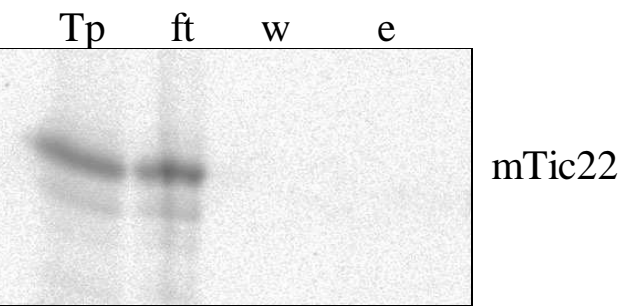

pMGD1

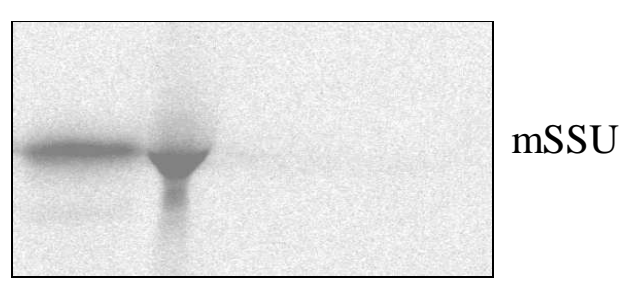

$\mathrm{pSSU}$

Figure 25. pTic22 and pMGD1 interact with the soluble domain of the receptor protein Toc34. A. For each separate experiment $300 \mu \mathrm{M}$ of overexpressed soluble receptor Toc34 $\Delta \mathrm{TM}$ was coupled to $10 \mu \mathrm{l} \mathrm{Ni-NTA}$ matrix and preloaded with $1 \mathrm{mM}$ GTP. Ni-NTA matrix without bound Toc $34 \Delta \mathrm{TM}$ was used as the negative control. ${ }^{35} \mathrm{~S}$-pTic22, ${ }^{35} \mathrm{~S}$-pMGD1 and ${ }^{35} \mathrm{~S}$-pSSU were added to the column in binding buffer and incubated for 45 minutes. The flow through after incubation (ft), the third wash of the matrix $(\mathrm{w})$ and the elution with $250 \mathrm{mM}$ imidazole (e) were analyzed by SDS-PAGE. Tp represents $1 / 10$ of the translation product used in each experiment. B. Control experiment using ${ }^{35} \mathrm{~S}-$ mTic22 and ${ }^{35} \mathrm{~S}-\mathrm{mSSU}$ in the previously described reaction.

To confirm the observed results another approach to test the interaction between the preproteins and the receptor GTPase Toc34 $4 \mathrm{TM}$ has been used. Freshly overexpressed and purified Toc34 $\Delta \mathrm{TM}$ was coupled to $\mathrm{Ni}-\mathrm{NTA}$ column by the interaction of $\mathrm{Ni}^{2+}$ and the His-tag on the C-terminus of the protein. The protein was preloaded with $1 \mathrm{mM} \mathrm{GTP}$ in the binding buffer (see section 5.7). Subsequently, radioactively labelled translation products of pTic22, pMGD1 or pSSU were applied to the column, incubated for 45 minutes, washed, and Toc34 $\triangle \mathrm{TM}$ with bound preproteins was eluted by $250 \mathrm{mM}$ imidazole and analyzed by SDS-PAGE. As controls, Ni-NTA matrix without bound Toc34 $\Delta \mathrm{TM}$, as well as mature forms of tested proteins (lacking the presequence) were used. The Toc34 $\Delta \mathrm{TM}$ binding experiment has shown that both pTic22 and pMGD1, as well as the control protein pSSU, bind specifically to the Toc34 $\triangle \mathrm{TM}$ GTPase (Figure $25 \mathrm{~A}$, lane 4). Binding to the empty N i-NTA matrix was not observed (Figure 25A, lane7). Binding of mTic22 and mSSU to Toc34 $\Delta \mathrm{TM}$ 
was tested as a control, using the same experimental setup. In both cases no binding could be observed (Figure 25B), indicating that Toc34 $\Delta \mathrm{TM}$ binds specifically to the presequences. Obtained results indicate that both pTic22 and pMGD1 use Toc34 as the initial receptor on their way into chloroplasts.

\subsection{MGD1 overexpression and antibody production}

For the purpose of overexpression and in vitro transcription, translation and import experiments, the original clone from Arabidopsis thaliana in pUNI51 vector was recloned into pET21d and pSP65 vectors. pMGD1 in pET21d vector failed to overexpress in any of the tested cell types. Also, when the first codons were replaced by those preferred by E.coli, this modification still did not influence the overexpression result. For the purpose of successful overexpression, the $\mathrm{N}$-terminal $321 \mathrm{bp}$ of the presequence have been removed and MGD1-P clone of $45.3 \mathrm{kDa}$ was created.

A

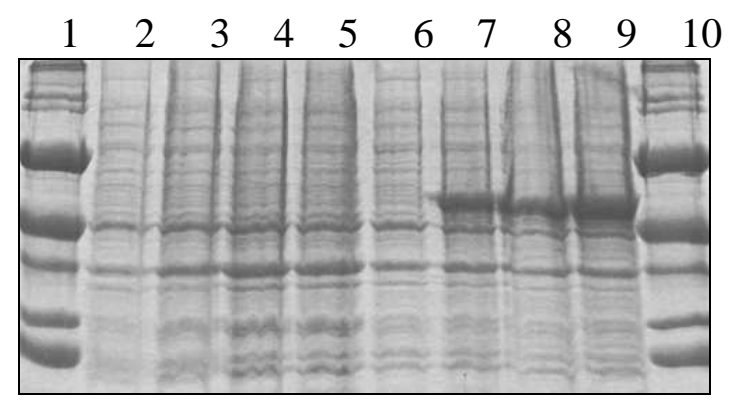

B

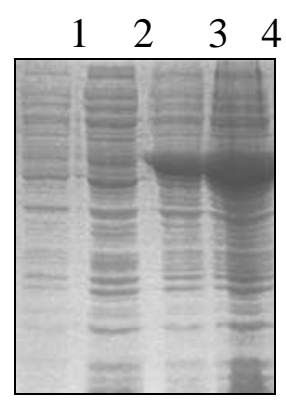

Figure 26. Overexpression of pMGD1 and MGD1-P clones. A. Overexpression of pMGD1 in pET21d vector (lanes 3-5) did not work. MGD1-P clone, lacking the presequence, has been constructed and successfully overexpressed in E.coli BL21(DE3) pRosetta cells (lanes 7-9). Lanes 2 and 6 represent control cells before induction with IPTG for pMGD1 and MGD1-P transformants, respectively. Lanes 3-5 and 7-9 represent samples taken after 1, 2 and 3 hours of overexpression. In lanes 1 and 10 is $15 \mu \mathrm{l}$ of the low molecular weight marker. B. After overexpression, cells were separated into the soluble (lanes 1 and 2 contain 5 and $20 \mu \mathrm{l}$ of sample) and in non-soluble fractions (lanes 3 and 4 contain 5 and $20 \mu \mathrm{l}$ of the sample).

Overexpression was performed as described in section 5.10.2 and typical results are shown in the Figure 26. Overexpression of MGD1-P was successful and the protein was recovered as inclusion bodies. Further purification was performed under denaturing conditions, using $8 \mathrm{M}$ urea. Because the protein could not be successfully purified on a Ni-NTA column using a pH- 
or imidazole-gradient for elution (Figures 27A and B), or HiTrap column (Figure 27C), further purification using cation-exchange chromatography column PorousS was performed (Figure 27D). This method provided pure fractions of MGD1-P protein, in addition to an extra band, slightly smaller in size. This band was successfully removed by subsequently applied gel-elution technique (Figure 27E). A sample purified by this method was concentrated to 1 $\mathrm{mg} / \mathrm{ml}$ and used for antibody production. Polyclonal antibodies were tested on purified protein MGD1-P and purified outer and inner envelope membranes from Pisum sativum (Figure 27F).

A

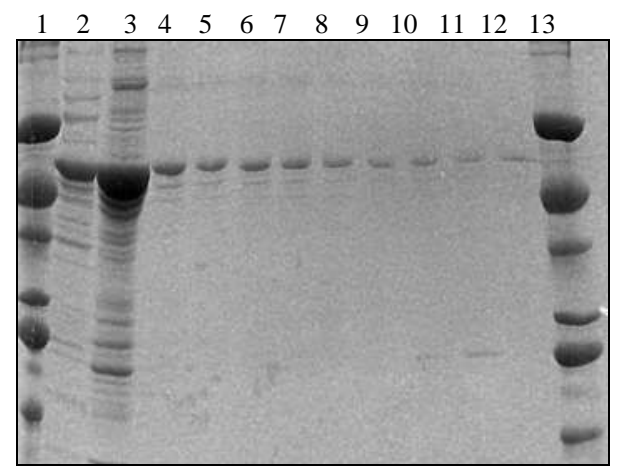

C

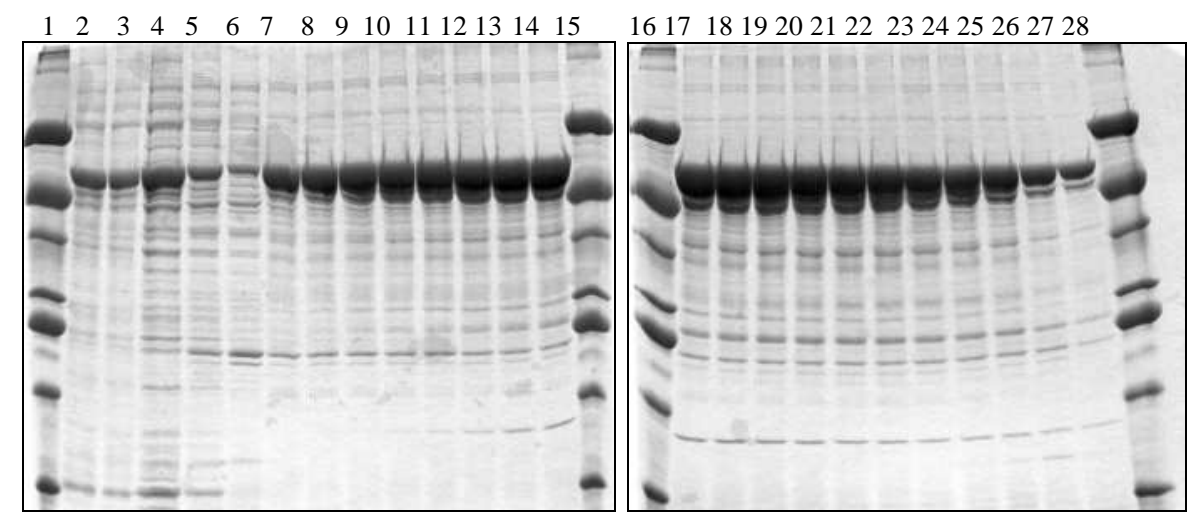

D

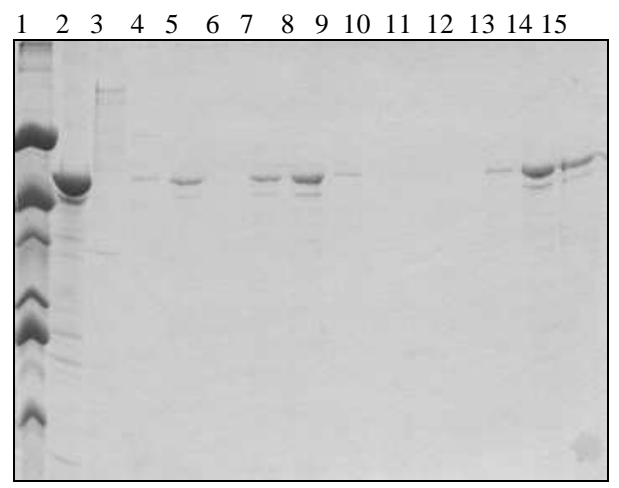

B

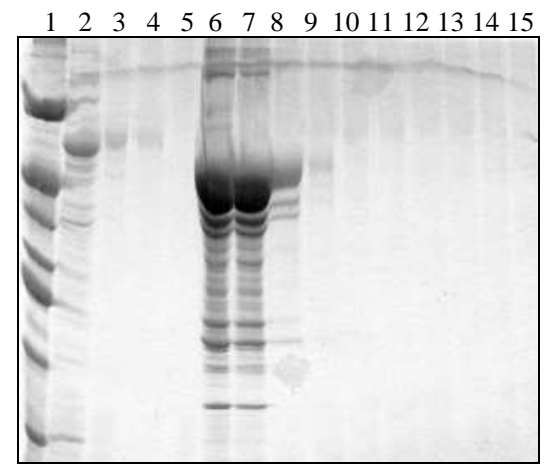

$\mathbf{E}$

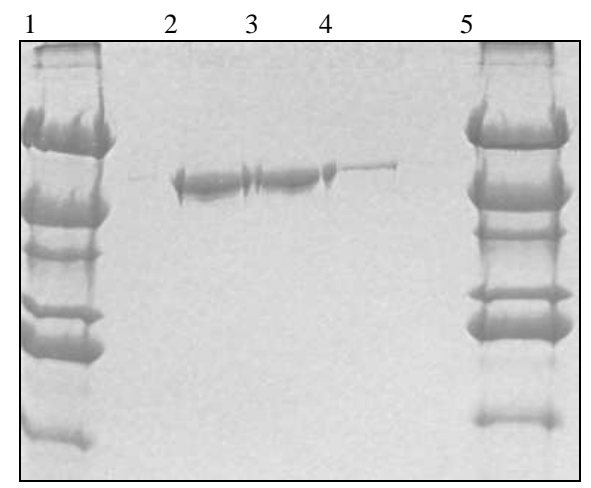




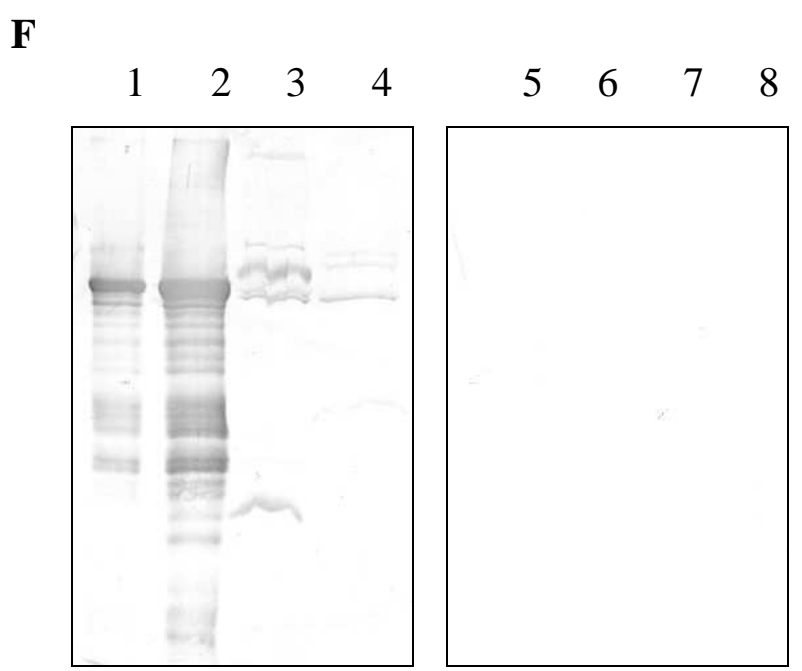

Figure 27: Purification of MGD1-P inclusion bodies. A. Purification on Ni-NTA column (His-tag), using a pH gradient elution. Lanes 1 and 13 represent $20 \mu \mathrm{l}$ of a low molecular weight marker. In lane 2 is $1 / 1000$ of the flow through, the column was washed with the buffer having a $\mathrm{pH} 6.3$ (lanes 3 and 4), and eluted by the buffers of $\mathrm{pH} 5.9$ (lanes 5-8) and $\mathrm{pH} 4.5$ (lanes 9-12). B. Purification on a Ni-NTA column using imidazole gradient elution. Lane 1 represents $20 \mu 1$ of a low molecular weight marker. In lane 2 is $1 / 1000$ of the flow through, the column was washed with $10 \mathrm{mM}$ imidazole (lanes 3-5) and eluted with increasing concentrations of imidazole (500 $\mathrm{mM}$ end concentration, lanes 6-15). C. Purification on HiTrap column (His-tag, Äkta) and elution by an imidazole gradient. The fractions under the peak area of the $\mathrm{A}_{280}$ absorption spectrum (higher protein amount) were tested. Lanes 2-3 represent the flow through, 4-6 the wash with $10 \mathrm{mM}$ imidazole and 7-27 the elution with an imidazole gradient 20-500 mM. Lanes $1,15,16$ and 28 indicate $20 \mu 1$ of a low molecular weight marker. D. Purification by cation-exchange chromatography using a PorousS column. Lane 1 represents $20 \mu \mathrm{l}$ of a low molecular weight marker. $20 \mu \mathrm{l}$ of the sample before purification were loaded in lane $2.30 \mu \mathrm{l}$ of the flow through after applying the sample on the column is in lane 3. 1/10 of different fractions collected in the peak intervals are shown in lanes 4-15. E. The additional lower band was removed from MGD1-P by gel elution (lanes 2-4). Lanes 1 and 5 indicate $20 \mu 1$ of low molecular weight marker. F. Testing of MGD1-P antibodies produced in rabbit. 0.65 and $1.3 \mu \mathrm{g}$ of the purified protein (lanes 1-2 and 5-6) and $20 \mu \mathrm{l}$ of the inner and outer envelopes (lanes 3-4 and 7-8) were analyzed by SDS-PAGE and western transfer, with subsequent immunodecoration with $\alpha$-MGD1-P antibodies (lanes 1-4) or with preimmune serum (lanes 5-8).

\subsection{Chemical crosslinking and immunoprecipitation}

To investigate the other components of the Toc-translocon with which pTic22 and pMGD1 interact on their way inside the chloroplasts, chemical crosslinking and immunoprecipitation using antibodies against Toc34, Toc75, Tic110 and the outer envelope protein that is not a component of the import translocon, OEP16, were performed. Protein precursors ${ }^{35} \mathrm{~S}$-pTic22, ${ }^{35} \mathrm{~S}$-pMGD1 and ${ }^{35} \mathrm{~S}$-pSSU were bound to the receptors on the chloroplast surface after performing incubation in the import mix for 8 minutes on ice, or after inhibiting import by addition of $\mathrm{NiSO}_{4}$ (see section 5.6.1.4). Chloroplasts were reisolated over a Percoll cushion and crosslinked using 0.5 mM DSP, as described in section 5.6.2.3. Crosslinked chloroplasts 
were lysed, centrifuged, and resulting membranes solubilized in $1 \%$ SDS.

Immunoprecipitation was performed by incubation of solubilized membranes with $\alpha$-Toc34, $\alpha$-Toc75(III), $\alpha$-Tic110 and $\alpha$-OEP16 antibodies (Figure 28A). Antibodies were collected using Protein A-Sepharose. The flow through after incubation with Protein A-Sepharose, the

A

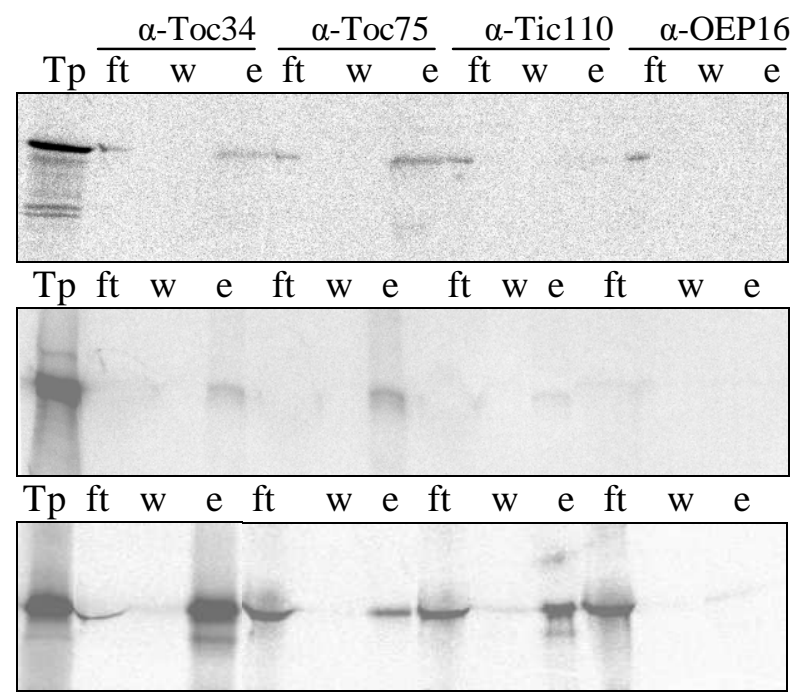

B

Tic22

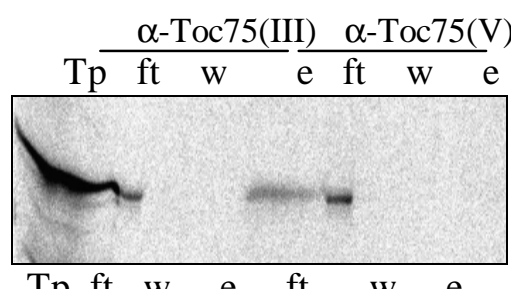

MGD1

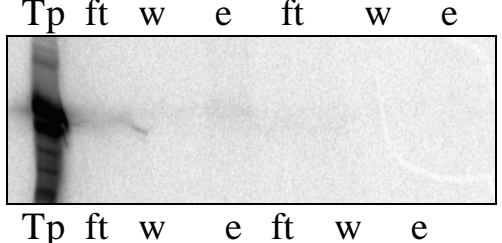

SSU

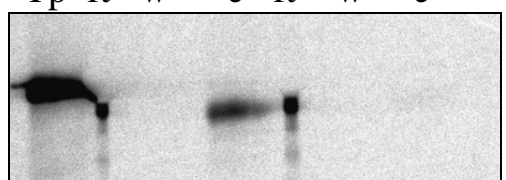

C

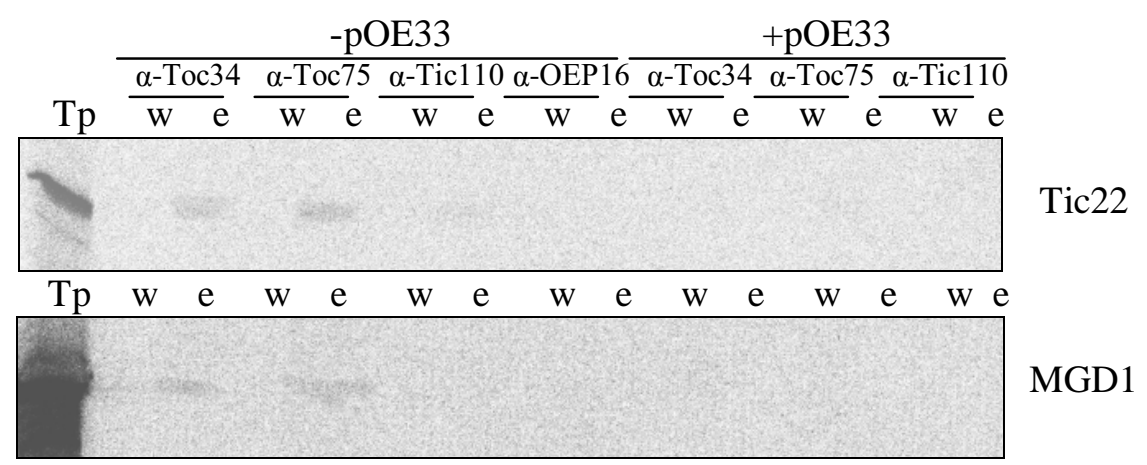

Figure 28. Chemical crosslinking and immunoprecipitation of pTic22 and pMGD1 to the major components of the translocation channel. A. ${ }^{35}$ S-pTic22, ${ }^{35}$ S-pMGD1 and ${ }^{35}$ S-pSSU were incubated with intact pea chloroplasts corresponding to $20 \mu \mathrm{g}$ chlorophyll for 8 minutes on ice. After reisolation on a Percoll cushion and subsequent washing, chloroplasts with bound precursor proteins were subjected to crosslinking using $0.5 \mathrm{mM}$ DSP. The immunoprecipitation was performed after the lysis of chloroplasts, centrifugation and solubilization of the membranes. Antibodies raised against Toc34, Toc75, Tic110 and OEP16 were used for 1 hour incubation at RT. Antibodies were collected using Protein A-Sepharose. 1/10 of the flow through after incubation with Protein A-Sepharose (ft), 1/10 of the third wash (w), and the elution with Laemmli sample buffer (e) were analyzed by SDS-PAGE. Tp indicates $1 / 10$ of the translation product used for each experiment. B. Crosslinking and immunoprecipitation were performed under the same conditions, using antibodies against two Toc75 isoforms: Toc75(III) and Toc75(V). C. Crosslinking and immunoprecipitation were performed in the absence or presence of $10 \mu \mathrm{M}$ pOE33 in the import mixture. 
third wash, and the elution with Laemmli sample buffer were analyzed by SDS-PAGE. pTic22 and pMGD1 were crosslinked to Toc34, Toc75 and to a lesser extent to Tic110 (Figure 28A).

It has been proposed that different isoforms of Toc and Tic components found in Arabidopsis join together to form different complexes with different preprotein specificity. It is believed that Toc33, Toc159, Toc75(III), Toc64(III), Tic22(IV), Tic110 and Tic40 join to form one type of the translocation complex, probably involved in the import of photosynthetic proteins; and on the other hand Toc34, Toc132 or Toc120, Toc75(III), Tic22(III) and Tic20(I) form the other type, probably involved in the import of the housekeeping genes (Vojta et al., 2004). Import through Toc75(V) has not been reported yet. To investigate whether Tic22 and MGD1 use the standard import pathway, comparison of crosslinking and immunoprecipitation between Toc75(III) and Toc75(V) isoforms have been tested (Figure 28B). Tic22 and MGD1 were found co-immunoprecipitated with Toc75(III) and not with Toc75(V). A control experiment using pSSU showed the same result. Also, crosslinking and immunoprecipitation were performed after binding of precursor proteins to the chloroplast surface in the presence or absence of $10 \mu \mathrm{M}$ pOE33 (Figure 28C). The lack of a signal after chemical crosslinking and immunoprecipitation with antibodies raised against the components of the outer and inner envelope translocons proves that pOE33 in the import mix interfered with the binding of pTic22 and pMGD1 to the chloroplast surface. Acquired result indicates once again that all tested proteins use the same receptors at initial steps of their import. After binding to Toc34 (in pea) or Toc33 (in Arabidopsis), Tic22 and MGD1 are most likely transferred to the Toc159 and subsequently to the Toc 75 translocation channel (Toc75(III)) on their way to the intermembrane space. From there Tic22 is probably released directly to the surface of the inner envelope, where it interacts with Tic110 and Tic20 (Kouranov et al., 1998), forming the Tic-translocon. MGD1 requires more energy for its import into the chloroplasts, as observed by performing ATP- and time-scale import experiments. MGD1 probably transfers through the Tic-translocon to the chloroplast stroma, where it is subsequently processed by the stromal processing peptidase. This translocation through the Tic-complex is most likely partial, because no soluble intermediates of MGD1 (nor of Tic22) have been observed by the experiments performed here. After processing, MGD1 has to reach its final destination in the intermembrane space. 


\subsection{Localization of MGD1}

Tic22 is known to be located in the chloroplastic intermembrane space between the outer and the inner envelope membranes, and peripherally associated with the outer face of the inner membrane. For MGDG synthases from different plant organisms enzymatic activity has been investigated and it was proposed that the enzyme associates either with the outer envelope (cucumber, Shimojima et al., 1997), the inner envelope (Arabidopsis, spinach, Miège et al., 1999, Maréchal et al., 2000) or both envelope membranes (pea, Cline and Keegstra, 1983). Up to date, the question of the exact localization of the MGDG synthases has not been clarified. To resolve this problem, import of the Arabidopsis protein into chloroplasts isolated from Arabidopsis, or pea isoform into the pea chloroplasts had to be performed.

Import of MGD1 into intact Arabidopsis chloroplasts did not show interpretable results, and MGDG synthase from pea has not been characterized yet. For that reason import into pea chloroplasts was performed using the Arabidopsis protein atMGD1. Mature MGD1 was visible in two forms after import into pea chloroplasts (described in section 6.2). To investigate whether those two forms of MGD1 exist in vivo in Arabidopsis plants, the mature form of MGD1 imported into pea chloroplasts was compared with the one from mixed envelopes isolated from $A$. thaliana.

Import of MGD1 has been performed under the standard conditions, and after chloroplast reisolation and thermolysin treatment samples were solubilized in $6 \mathrm{M}$ urea. The resulting samples were analyzed by SDS-PAGE and subjected to a western transfer and immunodecoration with $\alpha$-MGD1-P (Figure 29A). At the end, the membrane was exposed on a film. The film (Figure 29B) and the membrane were overlayed after developing (Figure 29C). After comparing the samples treated with urea (Figure 29, lanes 2-11), it could be observed that the size and the shape of the mature form of MGD1 found in Arabidopsis envelopes correspond to the mature form of this protein determined by import into pea chloroplasts (compare lanes 10 and 8 and 9 in Figure 29C). Samples that were not treated with urea (Figure 29C, lanes 12-14) showed the same result: two distinct bands were visible in Arabidopsis envelopes, corresponding in size and shape to those derived by import into pea chloroplasts. These results indicate that the situation observed after in vitro imports of MGD1 into chloroplasts corresponds to the in vivo situation in A. thaliana and heterologous import of atMGD1 into pea chloroplasts gives rise to the mature product of the same size and shape as found attached to the Arabidopsis envelopes. 
A

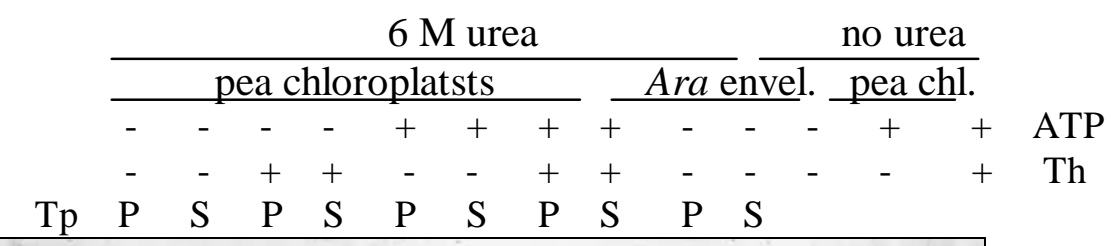

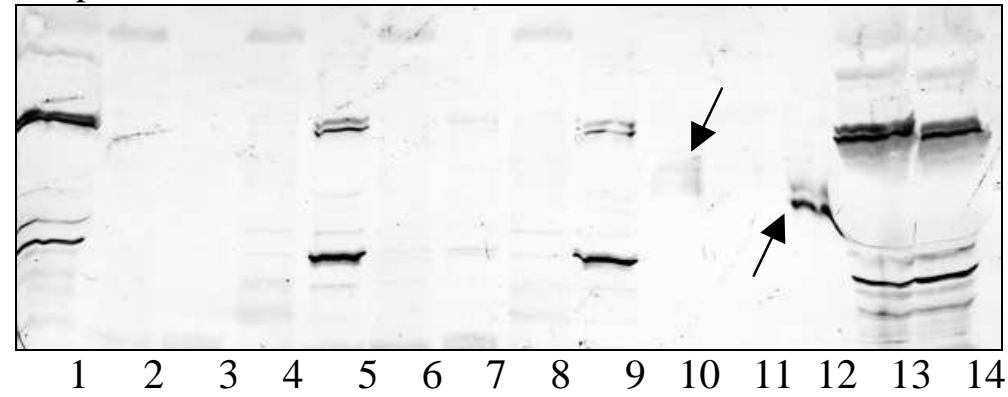

membrane

B

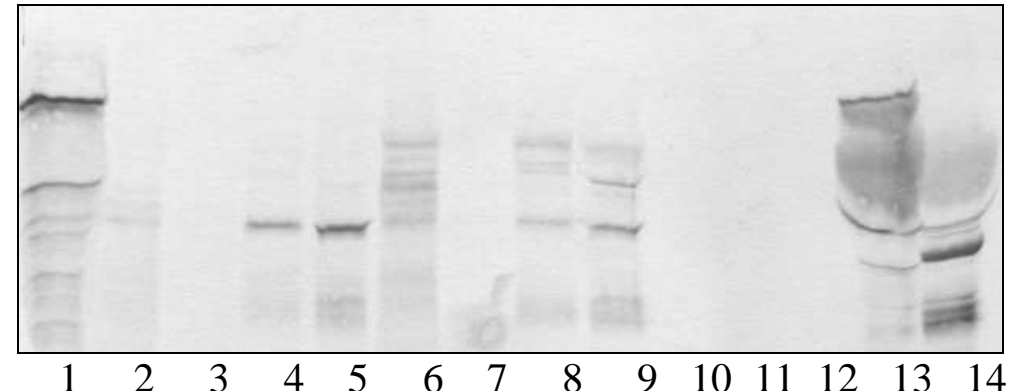

film

C

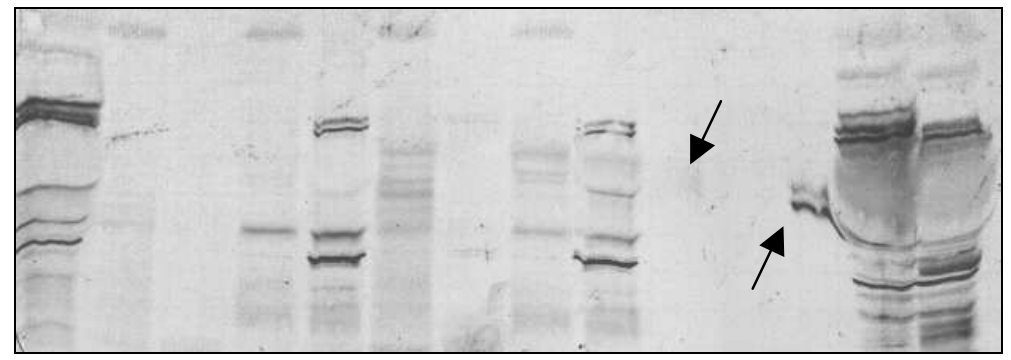

overlay

Figure 29. Size comparison of MGD1 between Arabidopsis envelopes and two forms visible after import into pea chloroplasts. ${ }^{35} \mathrm{~S}-\mathrm{MGD} 1$ was incubated with intact pea chloroplasts corresponding to $20 \mu \mathrm{g}$ chlorophyll in a standard import reaction for 15 minutes at $25^{\circ} \mathrm{C}$. The reaction was performed in the presence (lanes 6-9, 13 and 14) or the absence (lanes 2-5) of $3 \mathrm{mM}$ ATP. After reisolation on a Percoll cushion and subsequent washing, some of the samples were subjected to the thermolysin treatment (lanes $4,5,8,9,14$ ). Afterwards, chloroplasts were lysed and treated with $6 \mathrm{M}$ urea for 20 minutes on RT (lanes 2-11). After centrifugation at 256,000xg for 10 minutes at $4^{\circ} \mathrm{C}$, pellet (P) consisting of chloroplastic membranes and supernatant (S) were separated and analyzed by SDS-PAGE. A part of the sample was not treated by urea (lanes 12-14). Western transfer to a nitrocellulose membrane was performed, followed by immunodecoration using $\alpha$-MGD1-P antibodies. $\mathrm{Tp}$ indicates $1 / 10$ of the translation product used for the import reaction. A. Signals observed after the immunodecoration of the nitrocellulose membrane with $\alpha$-MGD1-P antibody. B. Radioactive signals observed after exposure of immunodecorated nitrocellulose membrane on an X-ray film. C. Overlay of the x-ray film over the nitrocellulose membrane. Two forms of MGD1, observed in Arabidopsis envelopes, which correspond to the mature forms obtained by the import experiment, are indicated by the arrows.

Proteins that remain in the membrane fraction after treatment of membranes with $0.1 \mathrm{M}$ $\mathrm{Na}_{2} \mathrm{CO}_{3} \mathrm{pH} 11.5$ or $6 \mathrm{M}$ urea are probably integral membrane proteins. After treatment of MGD1 with $0.1 \mathrm{M} \mathrm{Na}_{2} \mathrm{CO}_{3}$, most of the precursor remained in the membrane fraction, which was specially clear in the samples imported under the presence of $3 \mathrm{mM}$ ATP (Figure 30, 
lanes 6-9). MGD1 degradation products derived from thermolysin posttreatment were found in the soluble fraction. Treatment of chloroplasts with $6 \mathrm{M}$ urea resulted in the same effect. After the treatment, the protein was found in the membrane fraction (Figure 30, lanes 12-19), and only after thermolysin digestion a portion of the imported form was found to be soluble (Figure 30, lanes 15 and 19). The observed results were surprising, since MGD1 was predicted to be just associated to either the outer or the inner chloroplast envelope membrane. Only a weak association of MGDG synthases with envelope membranes by electrostatic interactions has been proposed (Shimojima et al., 1997, Maréchal et al., 2000). The results obtained here suggest that the interaction might be much stronger than speculated.

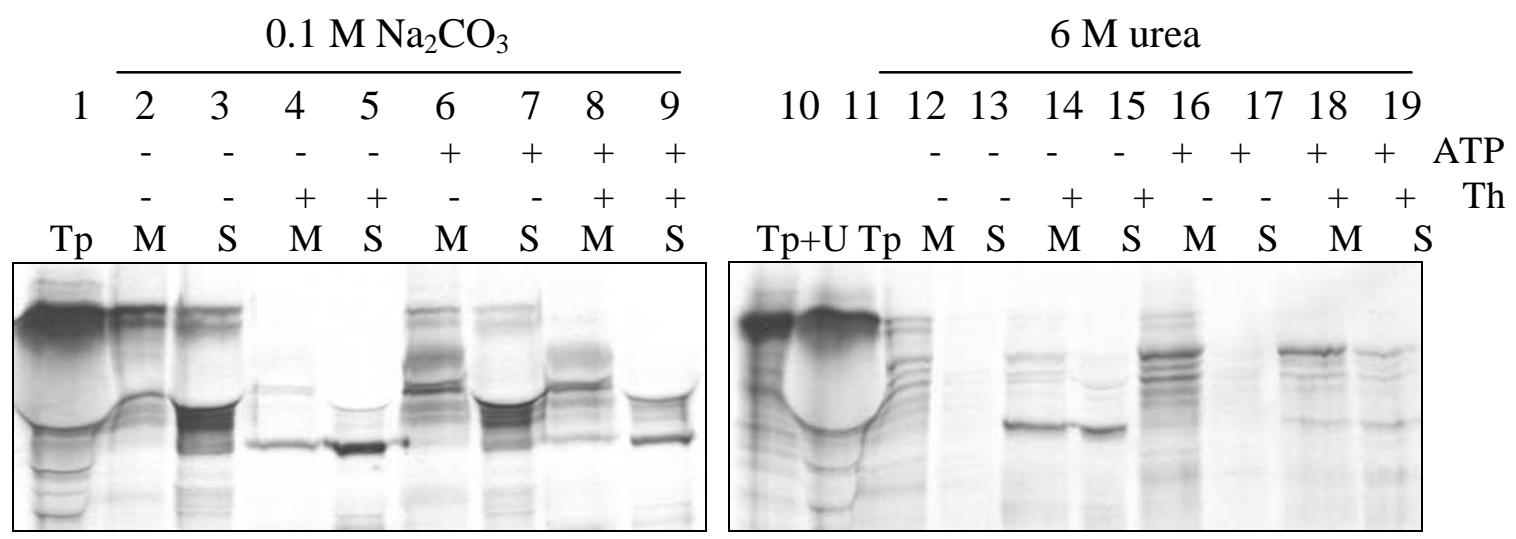

Figure 30. Extraction of MGD1 by $0.1 \mathrm{M} \mathrm{Na}_{2} \mathrm{CO}_{3}$ and $6 \mathrm{M}$ urea, after import into chloroplasts. After import of ${ }^{35} \mathrm{~S}-\mathrm{MGD}$, chloroplasts were treated with $0.1 \mathrm{M} \mathrm{Na}_{2} \mathrm{CO}_{3} \mathrm{pH} 11.5$ (lanes 2-9) or $6 \mathrm{M}$ urea (lanes 12-19) and separated into the membrane (M, lanes $2,4,6,8,12,14,16,18)$ and soluble fractions (S, lanes 3, 5, 7, 9, 13, 15, 17, 19). Import was performed in the absence (lanes 2-5, 12-15) or presence (lanes 6-9, 16-19) of $3 \mathrm{mM}$ ATP. After import chloroplasts were either treated with thermolysin (lanes 4, 5, 8, 9, 14, 15, 18, 19) or not (lanes 2, 3, 6, 7, 12, 13, 16, 17). Lanes 1 and 11 indicate $1 / 10$ of the translation product used for the import and lane 10 the translation product treated with urea.

To test the extent of an association of MGDG synthase to the membrane, extraction of isolated chloroplast envelopes from pea was performed. $6 \mathrm{M}$ urea, $0.1 \mathrm{M} \mathrm{Na}_{2} \mathrm{CO}_{3} \mathrm{pH} 11.5$ and $1 \mathrm{M} \mathrm{NaCl}$ were used to treat inner and outer envelope membranes corresponding to 20-30 $\mu \mathrm{g}$ protein for 20 minutes at RT (Figure 31). After the treatment, envelopes were centrifuged and the resulting pellet and supernatant were analyzed by SDS-PAGE and western transfer. $\alpha$-MGD1-P antibodies were used for immunodecoration of the nitrocellulose membrane after western-transfer and $\alpha$-Tic22, $\alpha$-Tic110 and $\alpha$-Toc75 were used as the controls for envelope purity and as the extraction control. 
In Pisum sativum, most of the MGDG synthase was found associated to the outer envelope (this finding corresponds to the results from Cline and Keegstra (1983), who localized the activity of MGDG synthase from pea to the outer envelope membrane) (Figure 31, lanes 13 and 14). Treatment with $0.1 \mathrm{M} \mathrm{Na}_{2} \mathrm{CO}_{3} \mathrm{pH} 11.5$ and $1 \mathrm{M} \mathrm{NaCl}$ gave similar results using either of the envelopes (Figure 31, compare lanes 3-6 and 9-12). The only difference was a small amount of protein detected in the soluble phase after treatment of the outer envelope vesicles (Figure 31, lanes 10 and 12 compared to 4 and 6). The fact that MGDG synthase from pea was not extracted from a membrane even by $1 \mathrm{M} \mathrm{NaCl}$ indicates that the interaction between this protein and the membrane is achieved by relatively strong electrostatic interactions. In contrast, treatment with $6 \mathrm{M}$ urea, gave different results for the tested envelopes. The portion of the protein associated with the inner envelope membrane was not extracted by $6 \mathrm{M}$ urea (Figure 31, lanes 1 and 2), and the portion associated to the outer envelope was found mostly in the soluble fraction (Figure 31, lanes 7 and 8).
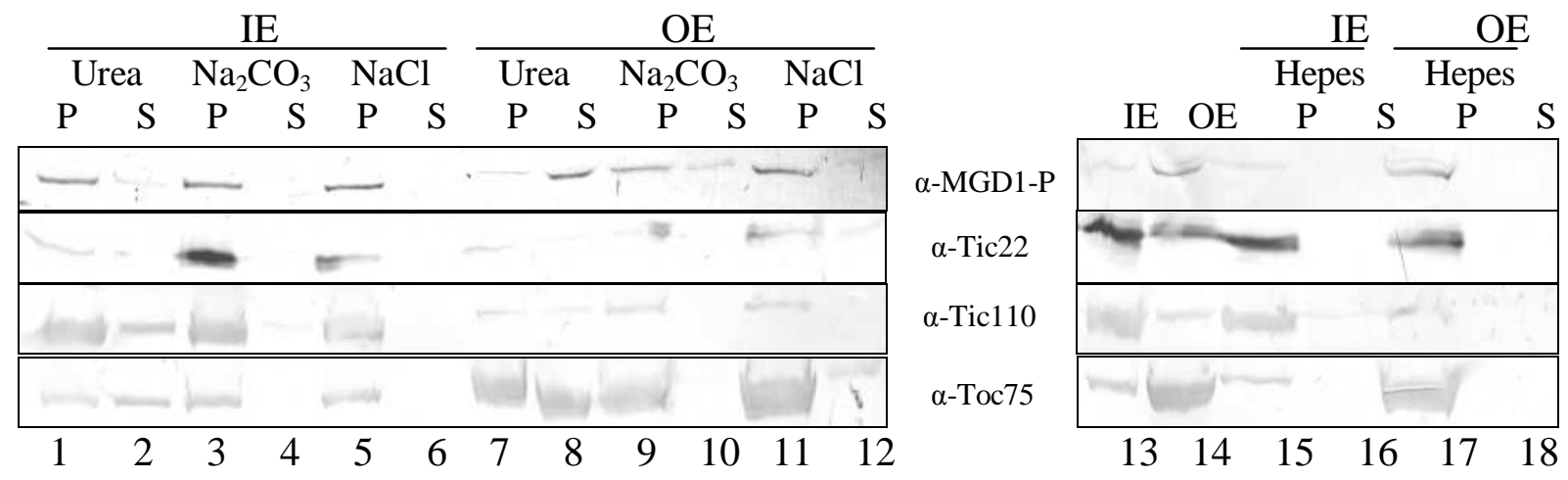

Figure 31. Extraction of MGDG synthase from inner and outer envelope vesicles from pea by 0.1 $\mathrm{M} \mathrm{Na}_{2} \mathrm{CO}_{3}, 6 \mathrm{M}$ urea and $1 \mathrm{M} \mathrm{NaCl}$. Chloroplast envelopes were isolated as described in section 5.3. Afterwards they were pelleted at $256,000 \times \mathrm{xg}$ for 10 minutes at $4^{\circ} \mathrm{C}$ and resuspended in either $0.1 \mathrm{M}$ $\mathrm{Na}_{2} \mathrm{CO}_{3} \mathrm{pH} 11.5$ (lanes 3, 4, 9, 10), $6 \mathrm{M}$ urea (lanes 1, 2, 7, 8), $1 \mathrm{M} \mathrm{NaCl}$ (lanes 5, 6, 11, 12) for 20 minutes on RT, or in $10 \mathrm{mM}$ Hepes/KOH pH 7.6 (lanes 15-18) for 20 minutes on ice, followed by centrifugation at $256,000 \times \mathrm{xg}$ for 10 minutes at $4^{\circ} \mathrm{C}$. The pellet and the supernatant were analyzed by SDS-PAGE and western-blotting. $\alpha$-MGD1-P, $\alpha$-Tic22, $\alpha$-Tic110 and $\alpha$-Toc75 were used for immunodecoration of the nitrocellulose membrane after western-transfer. The distribution of the components between the two envelope membranes can be estimated by comparing lanes 13 and 14 .

The experiment was repeated twice more using envelopes produced in different isolation runs, and the results were confirmed in both cases. These results show clearly that the MGDG synthase from pea associates with strong interactions to the inner envelope membrane, and just weakly attaches to the outer envelope membrane. 


\section{Discussion}

The aim of this thesis was to investigate import properties of Tic110, Tic22 and MGD1 into isolated pea chloroplasts to define the pathway that each of those proteins uses for its translocation to the final destination: the inner envelope membrane for Tic110 or the intermembrane space for Tic22 and MGD1.

\subsection{Import pathway of Tic110 into the inner envelope membrane of chloroplasts}

By the mutual exchange of transit sequences between Tic110 and pSSU it has been shown that Tic110 utilizes the general chloroplast import pathway (Lübeck et al., 1997). But only when the hybrid protein pSSU-Tic110N-mSSU was used in import experiments, a soluble intermediate in the translocation pathway was clearly visible. The construct pSSU-Tic110N-mSSU was completely imported into chloroplast stroma, from which it could be recovered as a soluble, processed Tic110N-mSSU (Lübeck et al., 1997). Soluble Tic110N-mSSU was shown to enter a re-export pathway, which resulted in its insertion into the inner envelope membrane, and in the extrusion of large portions of the protein into the intermembrane space. Lübeck et al. (1997) have proposed that chloroplasts possess a protein re-export machinery for inner envelope proteins in which soluble stromal components interact with the membrane-localized translocation machinery. It was proposed that this translocation machinery could export a large polypeptide chain across the membrane into the intermembrane space. The components of the export machinery are unknown at the present time.

After processing of pSSU-Tic110N-mSSU in the stroma, Tic110N-mSSU is translocated to the inner envelope, as the mature form. From this it could be concluded that an export signal is not present in the transit sequence, but an internal targeting information is necessary. Since Tic110-mSSU successfully inserted into the inner envelope membrane it could be concluded that a re-export signal is localized in the N-proximal part of Tic110 (putative membrane anchor region), and is sufficient for targeting to the inner membrane and proper insertion. The soluble intermediate form of the full length Tic110 has not been observed in the stroma previously due to the low translation and import efficiencies of this protein (Lübeck et al., 1997). The translation efficiency of Tic110 has been improved in this 
work by the addition of six methionines at the C-terminal end of the protein to enhance the intensity of the radioactive signal after translation. Tic110 followed the same import pathway, as observed previously for pSSU-Tic110N-mSSU. The processed form of Tic110 was found soluble in the stroma and, following how the import proceeds on a time scale, its re-export to the membrane was clearly visible. It was observed that just a portion of the protein (maximum $50 \%$ for Tic $110 \mathrm{~N}-\mathrm{mSSU}$ and $15 \%$ for mTic110) was found soluble, while the larger portion of the mature protein was always recovered in the membrane fraction. From these experiments it could be proposed that a fraction of Tic110N-mSSU or mTic110 is never released from the membrane, indicating that the parallel mechanisms for the import into the inner envelope might exist: stop-transfer and conservative sorting. However, it seems unlikely that a protein would use two different import mechanisms in vivo. Since the soluble stromal intermediate of Tic110 has been persistantly observed, it is possible that the re-export process is highly efficient and that only very little soluble intermediate accumulates in the stroma. Therefore, it seems that Tic110 uses conservative sorting for its translocation into chloroplasts.

Recently, Li and Schnell (2006) reported that pTic40 from A.thaliana is imported into chloroplasts and processed to an intermediate size form (iTic40) before insertion into the inner membrane. iTic40 is soluble and inserts into the inner envelope membrane from the stromal compartment. Together with the results obtained in this work it can be proposed that inner membrane proteins are first imported into the stroma and subsequently inserted into the inner envelope in a re-export mechanism.

It has been observed that the native psTic110 associates reversibly with Cpn60 (Hsp60 homologue, Kessler and Blobel, 1996) and Hsp93 (chloroplast ClpC homologue, Akita et al., 1997, Nielsen et al., 1997) chaperones of the chloroplast stroma. Kessler and Blobel have proposed that Tic110 acts as a docking site for molecular chaperones at the inner membrane. Akita et al. observed that significant quantities of ClpC were immunoprecipitated with antibodies against Tic110 and proposed that ClpC fills the role played by $\mathrm{mtHsp70}$ during protein translocation into mitochondria. Nielsen et al. first observed that the precursor, but not the mature form of SSU, was detected in the $\alpha$-ClpC immunoprecipitated fractions. It has been proposed that the association of ClpC was specific to precursors associated with the chloroplastic protein import apparatus. Also, complexes that co-immunoprecipitated with ClpC contained Tic110, Toc159, Toc75 and Toc34, regardless of whether precursor was 
present or not. Therefore, Nielsen et al. (1997) suggested that the interaction of Tic110 with $\mathrm{ClpC}$ and $\mathrm{Cpn60}$ is not necessarily bound to the chloroplast protein import.

In this work, the soluble stromal intermediates of mTic110 and Tic110N-mSSU were reisolated after import and co-immunoprecipitation with stromal chaperones Hsp93 (ClpC), Hsp70 and Cpn60 was performed. Co-immunoprecipitation with all tested chaperones indicate that Tic110 interacts with stromal chaperones during its own maturation, and not only as a component of the translocation machinery. Co-immunoprecipitation with Hsp93 was of the highest intensity, indicating that this chaperone might play a major role in helping Tic110 to reach the native conformation, and Hsp70 and to lesser extent Cpn60 might play an accessory role in this process. Membrane-bound fractions of mTic110 and Tic110N-mSSU also precipitated together with Hsp70 and Hsp93, though at a very low extent. In this case binding to Hsp93 was very weak (Figure 13B), which contradicts the above mentioned results from Akita et al. (1997), performed in vivo.

\subsection{Import pathway of intermembrane space protein Tic22}

Arabidopsis Tic22 (At4g33350) shows high homology to Tic22 from P. sativum and therefore it has been expected to import into intact pea chloroplasts in vitro. Indeed, although at a very low yield (3\%), atTic22 imported into isolated pea chloroplasts. Import of Tic22 is dependent on its N-terminal presequence (Figure 15), which is necessary and sufficient for targeting to the intermembrane space of chloroplasts. Import of Tic22 into chloroplasts required low amounts of externally added ATP. As a result of the protease treatment after import, both the precursor and mature forms of the protein remained protected from degradation to the same extent (mostly around 50\%, Figure 15). It appears that, although imported, not the entire population of radioactively labeled precursor protein was successfully processed to the mature form. This effect has been observed previously (Kouranov et al., 1999). With increasing ATP concentrations more of the imported precursor and mature forms remain protected from protease digestion, indicating that although low amounts of ATP are enough for initial import of Tic22, $1 \mathrm{mM}$ ATP is necessary to accomplish the maximal import rate (Figure 17C). The same has been observed by varying the time of import. A portion of Tic 22 was imported already on ice, but only after 10-15 minutes in the presence of $3 \mathrm{mM}$ ATP, a significant amount of imported protein remained protected from protease degradation (Figure 18). It was clearly shown that Tic22 needs ATP, and not GTP, for its import into chloroplasts (Figure 
17A). All these results point to the conclusion that import of Tic22 into the intermembrane space is relatively fast, but its processing is very slow and even after prolonged import duration the conversion from the precursor to the mature form never reaches more than around $20 \%$ in vitro.

In contrast to the stromal protein SSU and another investigated intermembrane space protein MGD1, Tic22 was not processed by the isolated stromal extract containing stromal processing peptidase (Figure 21A). This result, together with the low energy needs for its import, indicate that Tic22 might be imported through the outer envelope membrane, being immediately afterwards released to its final position in the intermembrane space. There, in the presence of higher ATP concentrations it could be stabilized and aquire the native conformation by interaction with intermembrane space chaperones. The question concerning its processing remains still unanswered. Mitochondria, like chloroplasts, are surrounded by two envelope membranes. Techniques have been developed to physically remove the mitochondrial outer envelope membrane. Mitoplasts, mitochondria of which the outer membrane has been selectively ruptured and/or dissolved, can be generated either by subjecting intact mitochondria to osmotic shock treatment (Daum et al., 1982) or by treating them with digitonin (Hartl et al., 1986). These two methods have been used successfully to study the localization and topology of mitochondrial inner envelope membrane proteins and the mechanism of mitochondrial protein import. Similar techniques to selectively remove the outer membrane of chloroplast envelopes are not yet available and the intermembrane space of chloroplasts has not been successfully isolated up to date. After the fractionation of chloroplasts it could be assumed that the stromal fraction contains a portion of the intermembrane space content. The stromal processing peptidase processed pSSU and pMGD1 with high efficiency. Since the stromal extract failed to process Tic22 it could be concluded that some other processing peptidase is required for processing of this protein. It is probable that Tic22 is processed in the intermembrane space of chloroplasts by a yet unknown peptidase or maybe by the same protease as iToc75 (Inoue et al., 2005). As mentioned in the introduction, Toc75 is processed by the SPP to the intermediate form (iToc75), which is cleaved to the mature Toc75 prior to insertion to the outer envelope, probably by the type I signal peptidase from the intermembrane space (Inoue et al., 2005).

Even when supposed that the intermembrane space content was isolated together with the stromal extract, it seems that an intermembrane space peptidase that would be responsible for processing of Tic22 was present there in too low amounts for successful processing to occur, or it could be associated to one of the envelope membranes and in this case was 
isolated with the membrane fraction. To check the second possibility the radioactively labelled Tic22 was incubated with mixed envelope vesicles from P. sativum in the presence of $0.5 \%$ DeMa in $20 \mathrm{mM}$ Hepes/KOH pH $8,0.5 \mathrm{mM} \mathrm{CaCl}_{2}$ and $0.5 \mathrm{mM} \mathrm{MgCl}_{2}$. Under the applied conditions no processing of Tic 22 could be observed by the mixed envelopes (data not shown), leaving the question where the processing of Tic22 takes place unanswered.

To investigate whether Tic22 needs the presence of thermolysin-sensitive components on the chloroplast surface for its import, chloroplasts were pretreated prior to import with the protease thermolysin that removes receptors exposed to the cytosol. Import of Tic22 into the chloroplasts treated this way was diminished to $40 \%$ (Figure 22), indicating that removed components are essential for binding and translocation of Tic22 into chloroplasts. Chloroplasts should be treated by such concentration of thermolysin that removes most of the surface exposed receptors, but in the same time does not disrupt significant amounts of chloroplasts. $1 \mathrm{mg}$ thermolysin per mg chlorophyll was applied in the experiments and led to around $30-40 \%$ chloroplast loss during the reisolation step. It could be considered that not all receptors were removed under these conditions, what could explain the residual $40 \%$ of successful import of Tic22. A similar result was shown by Kouranov et al. (1999). Their investigation had shown that Tic22 is dependent on thermolysin sensitive components on the chloroplast surface. On the basis of competition experiments the same group suggested a new pathway for targeting of Tic22 to the intermembrane space, distinct from known chloroplast targeting pathways. The competition experiments performed in this work argue against this proposal. Increasing concentrations of the competitor protein pOE33, known to use the Tocand Tic-translocon during its import into chloroplasts (Row and Gray, 2001), resulted in decreased import of Tic22. Even small quantities of pOE33 visibly inhibited Tic22 import, and the maximum of $10 \mu \mathrm{M}$ pOE33 used in import reaction resulted in more than $70 \%$ inhibition of Tic22 import (Figure 23). The observed results clearly indicate that pOE33 and Tic22 use the same pathway for their import into chloroplasts, namely the Toc-complex on the general import pathway.

These results were confirmed by the competition experiments using Toc $34 \Delta \mathrm{TM}$ and by crosslinking experiments. Toc34 $\Delta \mathrm{TM}$ added in import mix competed with the endogenous Toc34 for binding of Tic22 (Figure 24), indicating that Toc34 recognizes and interacts with Tic22 at the initial step of its import into chloroplasts. Interaction between the soluble domain of Toc34 and precursor protein Tic22, but not its mature form (Figure 25), has shown again the specificity of this interaction and confirmed previously shown results. Chemical crosslinking and immunoprecipitation experiments clearly demonstrate that Tic 22 binds to the 
chloroplast surface by interaction with Toc34, Toc159 (data not shown) and Toc75, the members of the Toc-translocon. In immunoprecipitation experiment, antibody against atToc75(III) isoform precipitated Tic22, in contrast to $\alpha$-Toc75(V). Crosslinking and immunoprecipitation, together with import competition experiments, indicate that Tic22 translocates through the Toc-complex to the intermembrane space. According to the low ATP-demands for its import, it seems that Tic22 is released from the Toc-complex to the intermembrane space of chloroplasts, where it reaches its final localization. Future experiments characterizing the exact site of processing, as well as investigation of the interaction of Tic22 with the components of the intermembrane space translocon, could clarify the events between the release from the Toc 75 translocation channel and aquiring its final conformation. Crosslinking of Tic22 to Tic110 has been observed to a certain extent, what could be explained by their interaction on the intermembrane side of the inner envelope, as components of the Tic-translocon. The observed interaction does not mean that Tic22 uses Tic110 for further translocation because the stromal protein SSU, which is known to transverse the Tic-translocation channel, was found to be immunoprecipitated at much higher extent with Tic110 during its import.

\subsection{Import and localization of MGD1 in the intermembrane space of chloroplasts}

MGDG synthases from different plants have been extensively studied (Maréchal et al., 1993, Shimojima et al., 1997, Miège et al., 1999, Maréchal et al., 2000), but the properties of their import pathways into chloroplasts have not been shown yet. Most of MGD1 import experiments in this work have been performed paralelly to Tic22, expecting some similarities in their import pathway on the basis of their intermembrane space localization. Hirsch and Soll (1995) have observed that $80 \mathrm{mM} \mathrm{K} \mathrm{KO}_{4}$ had increased import of the inner envelope membrane protein of $96 \mathrm{kDa}$, but diminished import rates of pSSU. Import of Tic22 in this research was not affected by the addition of $\mathrm{K}_{\mathrm{i}} \mathrm{PO}_{4}$ to any extent, in contrast to MGD1. Addition of $80 \mathrm{mM} \mathrm{K}_{\mathrm{i}} \mathrm{PO}_{4}$ to the import reaction enhanced the import rate of MGD1 (Figure 16B). The observation that the mature MGD1 appears in two forms and that both forms behaved the same in response to various import conditions led to the conclusion that they represent two forms of the same mature protein - a lipid associated (smeared) one and a part pressed by the large subunit of RubisCO. The fact that the lipid-associated form was hardly 
recognized by $\alpha$-MGD1-P antibody (Figure 29) agrees with the results observed by detection of MGD1 in whole chloroplasts, where MGD1 was hardly or not at all detectable (results not shown). The reason of hard detectability could be the low abundance of MGD1 in the plastid envelopes ( 1/1000 of total protein), as well as the lipid environment that could interfere with proper interaction between the antibody and the protein. When chloroplastic envelopes were tested for the presence of MGDG synthase by western blotting, envelope amount corresponding to at least $20-30 \mu \mathrm{g}$ total protein had to be used for MGDG synthase signal to be detectable.

The existence of the cleavable presequence was confirmed already by Awai et al. (2001), who immunodetected the mature polypeptide of MGD1 in the envelope of chloroplasts from Arabidopsis leaves after cleavage of its transit peptide. In this work, comparison of the sizes of MGD1 detected by western blotting in vivo with the mature forms visible after its import into chloroplasts confirmed that MGD1 contains an around $11 \mathrm{kDa}$ long presequence (as predicted by ChloroP) that targets the protein to chloroplasts where it is subsequently cleaved.

MGD1, like Tic22, is dependent exclusively on ATP for its import (Figure 17B). Although the processing of MGD1 has been observed even by performing import on ice, thermolysin protected forms started to appear after addition of $50 \mu \mathrm{M}$ ATP and complete import was accomplished using $1 \mathrm{mM}$ ATP (Figure 17C). MGD1 was processed by stromal extract (Figure 21). It was also imported more slowly than Tic22 in response to added ATP (Figures 18, 19C). The fast processing by stromal extract, the strong dependence on ATP for the complete translocation and the different ATP-dependence curve from Tic22 indicate that MGD1 most probably utilizes the import complexes from both envelope membranes. It translocates through the Toc-complex and, in contrast to Tic22, enters the Tic-translocon to enable the processing from the side of the stromal compartment to take place. To which extent MGD1 is exposed to the stroma during the processing could not be determined by the performed experiments. MGD1 is most probably never completely released from the inner envelope translocation channel, but rather exposes just its $\mathrm{N}$-terminal transit sequence to the SPP, and is subsequently pulled back towards the intermembrane space (as seems to be true for iToc75, destined for the outer envelope).

Thermolysin pretreatment of chloroplasts had an influence on MGD1 import, but to a lesser extent than on Tic22. Thermolysin pretreatment decreased the import of MGD1 to around $50 \%$, indicating that MGD1 is dependent on thermolysin sensitive receptors on the chloroplast surface for its import. Competition for import with pOE33 indicates that MGD1 
probably uses the Toc-translocon for its import into chloroplasts. As for Tic22, these results were confirmed by three further experiments: competition experiments using Toc $34 \Delta \mathrm{TM}$, interaction of the preprotein with Toc $34 \Delta \mathrm{TM}$ and crosslinking and immunoprecipitation with the major components of the Toc-translocon. Toc34 $\Delta \mathrm{TM}$ competed with the endogenous Toc34 for binding of MGD1 (Figure 24), indicating that Toc34 recognizes and interacts with pMGD1 at the initial step of its import into chloroplasts. Only the precursor protein interacted in vitro with the soluble receptor Toc34 $\mathrm{TM}$ (Figure 25). MGD1 was found crosslinked to Toc34, Toc75 and to Tic110. For the crosslinking experiment, conditions that allowed only binding to the chloroplast surface were used. Therefore the interaction between MGD1 and Tic110 had a weaker character. Crosslinking of late import intermediates (for example after 2-5 minutes of import at $25^{\circ} \mathrm{C}$ in the presence of $3 \mathrm{mM}$ ATP) could characterize import pathway of MGD1 further. Indeed, already by prolongation of the binding period, crosslinking to Tic110 could be observed (other components of the Tic-translocon have not been tested in this work). This result points to the previous proposal that MGD1 could use both Toc- and Tic-complexes for its import into chloroplasts.

Different localizations of MGDG synthases have been proposed in different plants. Enzymatic activity of MGDG synthase from spinach was found to be located on the inner membrane of the spinach chloroplast envelope (Block et al., 1983). Cline and Keegstra (1983) fractionated pea chloroplasts by flotation-centrifugation into thylakoids, soluble fraction and envelopes, that were further separated on a linear-density sucrose gradient. The gradient was assayed for galactosyltransferase, whose activity was detected in the outer envelope fraction and in one of the subfractions of the inner envelope, probably due to the contamination with the outer envelope. They proposed that MGDG synthase from pea associates to the outer envelope, but its presence from the inner envelope could not be excluded. In this work the localization of endogenous MGDG synthase in P. sativum have been investigated.

Treatment of the inner and outer envelope vesicles with $6 \mathrm{M}$ urea gave distinct extraction results for MGDG synthase. The portion of the protein associated to the inner envelope was not extracted by $6 \mathrm{M}$ urea (Figure 31, lanes 1 and 2), and the portion associated to the outer envelope was found mostly in the soluble fraction (Figure 31, lanes 7 and 8). Miège et al. (1999) analyzed the association of spinach MGD A with the inner envelope membrane by ionic and alkaline extractions. Although treatment of envelope membranes by $1 \mathrm{M} \mathrm{NaCl}$ had no effect on the enzyme association with the membrane, part of the protein was extracted by $0.1 \mathrm{M} \mathrm{Na}_{2} \mathrm{CO}_{3} \mathrm{pH} 11$ and all protein was extracted by $0.1 \mathrm{M} \mathrm{NaOH}$. These 
results suggested that soMGD A is neither a transmembrane nor a peripheral protein, but more likely is imbedded within one of the two leaflets of the inner envelope membrane. In this work $1 \mathrm{M} \mathrm{NaCl}$ had no influence on the association of pea MGDG synthase with the envelopes and $0.1 \mathrm{M} \mathrm{Na}_{2} \mathrm{CO}_{3} \mathrm{pH} 11.5$ extracted only a portion of the protein associated to the outer envelope. The observed results are similar to those obtained in spinach. It could be concluded that although preferentially associated to the outer envelope membrane, the portion of MGDG synthase from $P$. sativum interacts much stronger with the inner envelope, from where it could not be extracted either with $6 \mathrm{M}$ urea, or with $0.1 \mathrm{M} \mathrm{Na}_{2} \mathrm{CO}_{3}$.

Thus, although the localization of MGD1 in chloroplasts is not entirely clear, it could be shown in this work that pMGD1 uses the general import pathway and reaches, at least partly, the stromal compartment before ending up in the intermembrane space. Taken together, it has been clearly demonstrated that proteins of the inner envelope and intermembrane space partly use the general import pathway, but their route to their final destination diverges at the inner membrane. Therefore, for chloroplasts the same picture begins to emerge that has been shown for mitochondria - that different import pathways exist for distinct protein classes. 


\section{References}

Abad MS, Clark SE, Lamppa GK (1989) Properties of a chloroplast enzyme that cleaves the chlorophyll $\mathrm{a} / \mathrm{b}$ binding protein precursor: optimization of an organelle-free reaction. Plant Physiol 90:117-124

Akita M, Nielsen E, Keegstra K (1997) Identification of protein transport complexes in the chloroplastic envelope membranes via chemical cross-linking. J Cell Biol 136:983-994

Alefsen H, Waegemann K, Soll J (1994) Analysis of chloroplast protein import machinery. $J$ Plant Physiol 144:339-345

Arnon DJ (1949) Copper enzymes in isolated chloroplasts. Polyphenoloxidase in Beta vulgaris. Plant Physiol 24:1-15

Awai K, Maréchal E, Block MA, Brun D, Masuda T, Shimada H, Takamiya K-I, Ohta H, Joyard J (2001) Two types of MGDG synthase genes, found widely in both 16:3 and 18:3 plants, differentially mediate galactolipid syntheses in photosynthetic and nonphotosynthetic tissues in Arabidopsis thaliana. Proc Natl Acad Sci USA 98:10960-10965

Bauer J, Chen K, Hiltbunner A, Wehrli E, Eugster M, Schnell D, Kessler F (2000) The major protein import receptor of plastids is essential for chloroplast biogenesis. Nature 403:203-207

Becker T, Hritz J, Vogel M, Caliebe A, Bukau B, Soll J, Schleiff E (2004a) Toc12, a novel subunit of the intermembrane space preprotein translocon of chloroplasts. Mol Biol Cell 15:5130-5144

Becker T, Jelic M, Vojta A, Radunz A, Soll J, Schleiff E (2004b) Preprotein recognition by the Toc complex. EMBO J 23:520-530

Becker T, Qbadou S, Jelic M, Schleiff E (2005) Let's talk about... Chloroplast import. Plant Biol 7:1-14

Benning C, Ohta H (2005) Three enzyme systems for galactoglycerolipid biosynthesis are coordinately regulated in plants. J Biol Chem 280:2397-2400

Block MA, Dorne AJ, Joyard J, Douce R (1983) Preparation and characterization of membrane fractions enriched in outer and inner envelope membrane from spinach chloroplasts. J Biol Chem 258:13281-13286

Bölter B, Soll J, Hill K, Helmmer R, Wagner R (1999) A rectifying ATP-regulated solute channel in the chloroplastic outer envelope of pea. EMBO J 18:5505-5516

Bonner WM, Laskey RA (1974) A film detection method for tritium-labelled proteins and nucleic acids in polyacrylamide gels. Eur J Biochem 46:83-88

Bruce BD (1998) The role of lipids in plastid protein transport. Plant Mol Biol 38:223-246 
Caliebe A, Grimm R, Kaiser G, Lübeck J, Soll J, Heins L (1997) The chloroplastic protein import machinery contains a Rieske-type iron-sulfur cluster and a mononuclear iron-binding protein. EMBO J 16:7342-7350

Cavalier-Smith T (2000) Membrane heredity and early chloroplast evolution. Trends Plant Sci 5:174-182

Chen K, Chen X, Schnell DJ (2000) Mechanism of protein import across the chloroplast envelope. Biochem Soc Trans 28:485-491

Chen X, Smith MD, Fitzpatrick L, Schnell DJ (2002) In vivo analysis of the role of atTic20 in protein import into chloroplast. Plant Cell 14:641-654

Chigri F, Hörmann F, Stamp A, Stammers DK, Bölter B, Soll J, Vothknecht UC (2006) Calmodulin regulation of chloroplast protein translocation is mediated by calmodulin binding to Tic32. Proc Natl Acad Sci USA 103:16051-16056

Chou ML, Fitzpatrick LM, Tu SL, Budziszewski G, Potter-Lewis S, Akita M, Levin JZ, Keegstra K, Li HM (2003) Tic40, a membrane-anchored co-chaperone homolog in the chloroplast protein translocon. EMBO J 22:2970-2980

Chua NH, Schmidt GW (1978) Post-translational transport into intact chloroplasts of a precursor to the small subunit of ribulose-1,5-bisphosphate carboxylase. Proc Natl Acad Sci USA 75:6110-6114

Chung CT, Niemela SL, Miller RH (1989) One-step preparation of competent Escherichia coli: Transformation and storage of bacterial cells in the same solution. Proc Natl Acad Sci USA 86:2172-2175

Cline K, Henry R (1996) Import and routing of nucleus-encoded chloroplast proteins. Annu Rev Cell Dev Biol 12:1-26

Cline K, Keegstra K (1983) Galactosyltransferases involved in galactolipid biosynthesis are located in the outer membrane of pea chloroplast envelopes. Plant Physiol 71:366-372

Cline K, Werner-Washburne M, Lubben TH, Keegstra K (1985) Precursors to two nuclearencoded chloroplast proteins bind to the outer envelope membrane before being imported into chloroplasts. J Biol Chem 260:3691-3696

Daum G, Böhni PC, Schatz G (1982) Import of proteins into mitochondria. Cytochrome b2 and cytochrome c peroxidase are located in the intermembrane space of yeast mitochondria. $J$ Biol Chem 257:13028-13033

De Boer AD, Weisbeek PJ (1991) Chloroplast protein topogenesis: import, sorting and assembly. Biochim Biophys Acta 1071:221-253

Dörmann P, Benning C (2002) Galactolipids rule in seed plants. Trends Plant Sci 7:112-118

Emmanuelson O, Nielsen H, von Heijne G (1999) ChloroP, a neural network-based method for predicting chloroplast transit peptides and their cleavage sites. Protein Sci 8:978-984 
Friedman AL, Keegstra K (1989) Chloroplast protein import. Quantitative analysis of precursor binding. Plant Physiol 89:993-999

Fulgosi H, Soll J (2001) A gateway to chloroplasts - protein translocation and beyond. Plant Physiol 158:273-284

Gounaris K, Barber J (1983) Monogalactosyldiacylglycerol: The most abundant polar lipid in nature. Trends Biochem Sci 8:378-381

Gray JC, Row P (1995) Protein translocation across chloroplast envelope membranes. Trends Cell Biol 5:243-247

Hartl F-U, Schmidt B, Wachter E, Weiss H, Neupert W (1986) Transport into mitochondria and intramitochondrial sorting of the Fe/S protein of ubiquinol-cytochrome c reductase. Cell 47:939-951

Heins L, Collinson I, Soll J (1998) The protein translocation apparatus of chloroplast envelopes. Trends Plant Sci 3:56-61

Heins L, Soll J (1998) Chloroplast biogenesis: mixing the prokaryotic and the eukaryotic? Curr Biol 8:R215-217

Heins L, Mehrle A, Hemmler R, Wagner R, Kuchler M, Hormann F, Sveshnikov D, Soll J (2002) The preprotein conducting channel at the inner envelope membrane of plastids. EMBO J 21:2616-2625

Highfield PE, Ellis RJ (1978) Synthesis and transport of the small subunit of chloroplast ribulose bisphosphate carboxylase. Nature 271:420-424

Higuchi R (1990) Recombinant PCR. In PCR protocols: A guide to methods and applications, (Inns MA, Gelfand DH, Sninsky JJ, White TJ, eds.) Acad Press Inc, San Diego, pp. 177-183

Hinnah SC, Hill K, Wagner R, Schlicher T, Soll J (1997) Reconstitiution of a chloroplast protein import channel. EMBO J 16:7351-7360

Hinnah SC, Wagner R, Sveshnikova N, Harrer R, Soll J (2002) The chloroplast protein import channel Toc75: pore properties and interaction with transit peptides. Biophys $J$ 83:899-911

Hirsch S, Soll J (1995) Import of a new inner chloroplast envelope protein is greatly stimulated by potassium phosphate. Plant Mol Biol 27:1173-1181

Holmes DS, Quigley M (1981) A rapid boiling method for the preparation of bacterial plasmids. Anal Biochem Biophys 114:193-197

Hörmann F, Küchler M, Sveshnikov D, Oppermann U, Li Y, Soll J (2004) Tic32, an essential component in chloroplast biogenesis. J Biol Chem 279:34756-34762

Inaba T, Li M, Alvarez-Huerta M, Kessler F, Schnell D (2003) atTic110 functions as a scaffold for coordinating the stromal events of protein import into chloroplasts. J Biol Chem 278:38617-38627 
Inaba T, Alvarez-Huerta M, Li M, Bauer J, Ewers C, Kessler F, Schnell DJ (2005) Arabidopsis Tic110 is essential for the assembly and function of the protein import machinery of plastids. Plant Cell 17:1482-1496

Inoue K, Demel R, de Kruijff B, Keegstra K (2001) The N-terminal portion of the preToc75 transit peptide interacts with membrane lipids and inhibits binding and import of precursor proteins into isolated chloroplasts. Eur J Biochem 268:4036-4043

Inoue K, Baldwin AJ, Shipman RL, Matsui K, Theg SM, Ohme-Takagi M (2005) Complete maturation of the plastid protein translocation channel requires a type I signal peptidase. J Cell Biol 171:425-430

Jackson DT, Froehlich JE, Keegstra K (1998) The hydrophilic domain of Tic110, an inner envelope membrane component of the chloroplastic protein translocation apparatus, faces the stromal compartment. J Biol Chem 273:16583-16588

Jackson-Constan D, Akita M, Keegstra K (2001) Molecular chaperones involved in chloroplast protein import. Biochim Biophys Acta 1541:102-113

Jamet E, Parmentier Y, Durr A, Fleck J (1991) Genes encoding the small subunit of RUBISCO belong to two highly conserved subfamilies in Nicotianeae. J Mol Evol $33: 226-236$

Jarvis P (2004) Organellar proteomics: chloroplasts in spotlight. Curr Biol 14:317-319

Jarvis P, Dörmann P, Peto CA, Lutes J, Benning C, Chory J (2000) Galactolipid deficiency and abnormal chloroplast development in the Arabidopsis MGD synthase 1 mutant. Proc Natl Acad Sci USA 97:8175-8179

Jarvis P, Robinson C (2004) Mechanisms of protein import and routing in chloroplasts. Curr Biol 14:R1064-R1077

Jelic M, Sveshnikova N, Motzkus M, Horth P, Soll J, Schleiff E (2002) The chloroplast import receptor Toc34 functions as preprotein-regulated GTPase. Biol Chem 383:1875-1883

Joyard J, Block MA, Douce R (1991) Molecular aspects of plastid envelope biochemistry. Eur J Biochem 199:489-509

Joyard J, Teyssier E, Miège C, Berny-Seigneurin D, Maréchal E, Block MA, Dorne A-J, Rolland N, Ajlani G, Douce R (1998) The biochemical machinery of plastid envelope membranes. Plant Physiol 118:715-723

Keegstra K, Olsen LJ, Theg SM (1989) Chloroplastic precursors and their transport across the envelope membranes. Annu Rev Plant Physiol Plant Mol Biol 40:471-501

Keegstra K, Bruce B, Hurley M, Li H, Perry S (1995) Targeting of proteins into chloroplasts. Plant Physiol 93:157-162

Keegstra K, Cline K (1999) Protein import and routing systems of chloroplasts. Plant Cell 11:557-570 
Keegstra K, Jousif AE (1986) Isolation and characterization of chloroplast envelope membranes. Methods Enzymol 118:316-325

Kessler F, Blobel G, Patel HA, Schnell DJ (1994) Identification of two GTP-binding proteins in the chloroplast protein import machinery. Science 266:1035-1039

Kessler F, Blobel G (1996) Interaction of the protein import and folding machineries in the chloroplast. Proc Natl Acad Sci USA 93:7684-7689

Kessler F, Schnell D (2006) The function and diversity of plastid protein import pathways: A multilane GTPase highway into plastids. Traffic 7:1-10

Kikuchi S, Hirohashi T, Nakai M (2006) Characterization of the preprotein translocon at the outer envelope membrane of chloroplasts by blue native PAGE. Plant Cell Physiol 47:363-371

Ko K, Bornemisza O, Kourtz L, Ko ZW, Plaxton WC, Cashmore AR (1992) Isolation and characterization of a cDNA clone encoding a cognate $70-\mathrm{kDa}$ heat shock protein of the chloroplast envelope. J Biol Chem 267:2986-2993

Kobayashi K, Awai K, Takamiya K-I, Ohta H (2004) Arabidopsis type B monogalactosyldiacylglycerol synthase genes are expressed during pollen tube growth and induced by phosphate starvation. Plant Physiol 134:640-648

Kouranov A, Schnell DJ (1996) Protein translocation at the envelope and thylakoid membranes of chloroplasts. J Biol Chem 271:31009-31012

Kouranov A, Schnell DJ (1997) Analysis of the interactions of preproteins with the import machinery over the course of protein import into chloroplasts. J Cell Biol 139:1677-1685

Kouranov A, Chen X, Fuks B, Schnell DJ (1998) Tic20 and Tic22 are new components of the protein import apparatus at the chloroplast inner envelope membrane. $J$ Cell Biol 143:991-1002

Kouranov A, Wang H, Schnell DJ (1999) Tic22 is targeted to the intermembrane space of chloroplasts by a novel pathway. J Biol Chem 274:25181-25186

Kovacheva S, Bedard J, Patel R, Dudley P, Twell D, Rios G, Koncz C, Jarvis P (2005) In vivo studies on the roles of Tic110, Tic40 and Hsp93 during chloroplast protein import. Plant J 41:412-428

Küchler M, Decker S, Hörmann F, Soll J, Heins L (2002) Protein import into chloroplasts involves redox-regulated proteins. EMBO J 21:6136-6145

Laemmli UK (1970) Cleavage of structural proteins during the assembly of the head of bacteriophage T4. Nature 227:680-685

Li H-M, Moore T, Keegstra K (1991) Targeting of proteins to the outer envelope membrane uses a different pathway than transport into chloroplasts. Plant Cell 3:709-717 
Li M, Schnell DJ (2006) Reconstitution of protein targeting to the inner envelope membrane of chloroplasts. J Biol Chem 175:249-259

Lubben TH, Donaldson GK, Viitanen PV, Gatenby AA (1989) Several proteins imported into chloroplasts form stable complexes with the GroEL-related chloroplast molecular chaperone. Plant Cell 1:1223-1230

Lübeck J, Soll J, Akita M, Nielsen E, Keegstra K (1996) Topology of IEP110, a component of the chloroplastic protein import machinery present in the inner envelope memebrane. EMBO J $15: 4230-4238$

Lübeck J, Heins L, Soll J (1997) A nuclear-encoded chloroplastic inner envelope membrane protein uses a soluble sorting intermediate upon import into the organelle. J Cell Biol $137: 1279-1286$

Ma Y, Kouranov A, LaSala S, Schnell DJ (1996) Two components of the chloroplast protein import apparatus, IAP86 and IAP75, interact with the transit sequence during the recognition and translocation of precursor proteins in the outer envelope. J Cell Biol 134:315-327

Madueňo F, Napier JA, Gray JC (1993) Newly imported Rieske iron-sulfur protein associates with both cpn60 and hsp70 in the chloroplast stroma. Plant Cell 5:1865-1876

Maréchal E, Block MA, Joyard J, Douce R (1994) Kinetic properties of monogalactosyldiacylglycerol synthase from spinach chloroplast envelope membranes. $J$ Biol Chem 269:5788-5798

Maréchal E, Miège C, Block MA, Douce R, Joyard J (1995) The catalytic site of monogalactosyldiacylglycerol synthase from spinach chloroplast envelope membranes. $J$ Biol Chem 270:5714-5722

Maréchal E, Awai K, Block MA, Brun D, Masuda T, Shimada H, Takamiya K-I, Ohta H, Joyard J (2000) The multigenic family of monogalactosyl diacylglycerol synthases. Biochem Soc Transact 28:732-740

Marshall JS, DeRocher AE, Keegstra K, Vierling E (1990) Identification of heat shock protein hsp70 homologues in chloroplasts. Proc Natl Acad Sci USA 87:374-378

Marshall JS, Keegstra K (1992) Isolation and characterization of a cDNA clone encoding the major Hsp70 of the pea chloroplastic stroma. Plant Physiol 100:1048-1054

Martin W, Herrman RG (1998) Gene transfer from organelles to the nucleus: how much, what happens and why? Plant Physiol 118:9-17

Martin W, Rujan T, Richly E, Hansen A, Cornelsen S, Lins T, Leister D, Stoebe B, Hasegawa M, Penny D (2002) Evolutionary analysis of Arabidopsis, cyanobacterial and chloroplast genomes reveals plastid phylogeny and thousands of cyanobacterial genes in the nucleus. Proc Natl Acad Sci USA 99:12246-12251

May T, Soll J (1998) Positive charges determine the topology and functionality of the transmembrane domain in the chloroplastic outer envelope protein Toc34. J Cell Biol $141: 895-904$ 
May T, Soll J (2000) 14-3-3 proteins form a guidance complex with chloroplast precursor proteins in plants. Plant Cell 12:53-64

Melton DA, Krieg PA, Rebagliati MR, Maniatis T, Zinn K, Green MR (1984) Efficient in vitro synthesis of biologically active RNA and RNA hybridization probes from plasmids containing a bacteriophage SP6 promoter. Nucl Acid Res 12:7035-7056

Miège C, Maréchal E, Shimojima M, Awai K, Block MA, Ohta H, Takamiya K-I, Douce R, Joyard J (1999) Biochemical and topological properties of type A MGDG synthase, a spinach chloroplast envelope enzyme catalyzing the synthesis of both prokaryotic and eukaryotic MGDG. Eur J Biochem 265:990-1001

Mullet JE (1988) Chloroplast development and gene expression. Annu Rev Plant Physiol Plant Mol Biol 39:475-502

Moore T, Keegstra K (1993) Characterization of a cDNA clone encoding a chloroplasttargeted Clp homologue. Plant Mol Biol 21:525-537

Murata N, Kajiura H, Fujimura Y, Miyao M, Murata T, Watanabe A, Shinozaki K (1987) Partial amino acid sequences of the proteins of pea and spinach photosystem II complex. Prog Photosyn Res 1:701-704

Nada A, Soll J (2004) Inner envelope protein 32 is imported into chloroplasts by a novel pathway. J Cell Sci 117:3975-3982

Nielsen E, Akita M, Dávila-Aponte J, Keegstra K (1997) Stable association of chloroplastic precursors with protein translocation complexes that contain proteins from both envelope membranes and stromal Hsp100 molecular chaperone. EMBO J 16:935-946

Ohta H, Awai K, Takamiya K-I (2000) Glycerolipids of photosynthetic organisms - Their biosynthesis and evolutionary origin. Trends Glycosci Glycotechnol 12:241-253

Olsen LJ, Keegstra K (1992) The binding of precursor proteins to chloroplasts requires nucleoside triphosphates in the intermembrane space. J Biol Chem 267:433-439

Olsen LJ, Theg SM, Selman BR, Keegstra K (1989) ATP is required for the binding of precursor proteins to chloroplasts. J Biol Chem 264:6724-6729

Ongun A, Mudd JB (1968) Biosynthesis of galactolipids in plants. J Biol Chem 243:1558-1566

Pain D, Blobel G (1987) Protein import into chloroplasts requires a chloroplast ATPase. Proc Natl Acad Sci USA 84:3288-3292

Pain D, Kanwar YS, Blobel G (1988) Identification of a receptor for protein import into chloroplasts and its localization to envelope contact zones. Nature 331:232-237

Pinnaduwage P, Bruce BD (1996) In vitro interaction between a chloroplast transit peptide and chloroplast outer envelope lipids is sequence-specific and lipid class-dependent. $J$ Biol Chem 271:32907-32915 
Pohlmeyer K, Soll J, Steinkamp T, Hinnah H, Wagner R (1997) Isolation and characterization of an amino acid-selective channel protein present in the chloroplastic outer envelope membrane. Proc Natl Acad Sci USA 94:9504-9509

Pohlmeyer K, Soll J, Grimm R, Wagner R (1998) A high-conductance solute channel in the chloroplastic outer envelope from pea. Plant Cell 10:1207-1216

Pope B, Kent HM (1996) High efficiency 5 min transformation of Escherichia coli. Nucl Acid Res 24:536-537

Qbadou S, Tien R, Soll J, Schleiff E (2003) Membrane insertion of the chloroplast outer envelope protein, Toc34: constrains for insertion and topology. J Cell Sci 116:837-846

Qbadou S, Becker T, Mirus O, Tews I, Soll J, Schleiff E (2006) The molecular chaperone Hsp90 delivers precursor proteins to the chloroplast import receptor Toc64. EMBO J 25:1836-1847

Reumann S, Keegstra K (1999) The endosymbiotic origin of the protein import machinery of chloroplastic envelope membranes. Trends Plant Sci 4:302-307

Rial DV, Arakaki AK, Ceccarelli EA (2000) Interaction of the targeting sequence of chloroplast precursors with Hsp70 molecular chaperones. Eur J Biochem 267:6239-6248

Richter S, Lamppa GK (1999) Stromal processing peptidase binds transit peptides and initiates their ATP-dependent turnover in chloroplasts. J Cell Biol 147:33-44

Robinson C, Ellis RJ (1984) Transport of proteins into chloroplasts. Partial purification of a chloroplast protease involved in the processing of important precursor polypeptides. Eur $J$ Biochem 142:337-342

Rothen R, Thiess M, Schumann P, Boschetti A (1997) Import inhibition of poly(His) containing chloroplast precursor proteins by $\mathrm{Ni}^{2+}$ ions. FEBS Lett 403:15-18

Saiki RK, Gelfand DH, Stoffel B, Scharf SJ, Higuchi R, Horn GT, Mullis KB and Ehrlich HA (1998) Primer-directed enzymatic amplification of DNA with a thermo-stable DNA polymerase. Science 239:487-491

Salomon M, Fischer K, Fluegge U-I, Soll J (1990) Sequence analysis and protein import studies of an outer chloroplast envelope polypeptide. Proc Natl Acad Sci USA 87:5778-5782

Sambrook J, Fritsch EF, Maniatis T (1989) Molecular Cloning, A Laboratory Manual. Cold Spring Harbor Laboratory Press, New York

Schindler C, Hracky R, Soll J (1987): Protein transport in chloroplasts: ATP is a prerequisit. Z Naturforsch Sect C 42:103-108

Schleiff E, Soll J, Sveshnikova N, Tien R, Wright S, Dabney-Smith C, Subramanian C, Bruce BD (2002) Structural and guanosine triphosphate/diphosphate requirements for transit peptide recognition by the cytosolic domain of the chloroplast outer envelope receptor, Toc 34 . Biochemistry 41:1934-1946 
Schleiff E, Jelic M, Soll J (2003a) A GTP-driven motor moves proteins across the outer envelope of chloroplasts. Proc Natl Acad Sci USA 100:4604-4609

Schleiff E, Soll J, Küchler M, Kuhlbrandt W, Harrer R (2003b) Characterization of the translocon of the outer envelope of chloroplasts. J Cell Biol 160:541-551

Schnell DJ, Blobel G, Pain D (1990) The chloroplast import receptor is an integral membrane protein of chloroplast envelope contact sites. Cell Biol 111:1825-1838

Schnell DJ, Blobel G (1993) Identification of intermediates in the pathway of protein import into chloroplasts and their localization to envelope contact sites. J Cell Biol 120:103-115

Schnell DJ, Kessler F, Blobel G (1994) Isolation of components of the chloroplast protein import machinery. Science 266:1007-1012

Scott SV, Theg SM (1996) A new intermediate on the chloroplast protein import pathway reveals distinct translocation machineries in the two envelope membranes: energetics and mechanistic implications. J Cell Biol 132:63-75

Seedorf M, Waegemann K, Soll J (1995) A constituent of the chloroplast import complex represents a new type of GTP-binding protein. Plant J 7:401-411

Shimojima M, Ohta H, Iwamatsu A, Masuda T, Shioi Y, Takamiya K-I (1997) Cloning of the gene for monogalactosyldiacylglycerol synthase and its evolutionary origin. Proc Natl Acad Sci USA 94:333-337

Smith MD, Hiltbrunner A, Kessler F, Schnell DJ (2002) The targeting of the atToc159 preprotein receptor to the chloroplast outer membrane is mediated by its GTPase domain and is regulated by GTP. J Cell Biol 159:833-843

Soll J, Alefsen H, Boeckler B, Kerber B, Salomon M, Waegemann K (1992) Comparison of two different translocation mechanisms into chloroplasts. In "Membrane Biogenesis and Protein Targeting “(Neupert W, Lill R, eds.) pp.299-306

Soll J, Tien R (1998) Protein translocation into and across the chloroplastic envelope membranes. Plant Mol Biol 38:191-207

Soll J, Schleiff E (2004) Protein import into chloroplasts. Nat Rev Mol Cell Biol 5:198-208

Studier FW, Moffatt B (1986) Use of bacteriophage T7 RNA polymerase to direct selective high-level expression of cloned genes. J Mol Biol 189:113-130

Sveshnikova N, Soll J, Schleiff E (2000) Toc34 is a preprotein receptor regulated by GTP and phosphorylation. Proc Natl Acad Sci USA 97:4973-4978

Theg SM, Bauerle C, Olsen LJ, Selman BR, Keegstra K (1989) Internal ATP is the only energy requirement for the translocation of precursor proteins across chloroplastic membranes. J Biol Chem 264:6730-6736

Theg SM, Scott SV (1993) Protein import into chloroplasts. Trends Cell Biol 3:186-190 
Thompson JD, Higgins DG, Gibson TJ (1994) CLUSTAL W: improving the sensitivity of progressive multiple sequence alignment through sequence weighting, position-specific gap penalties and weight matrix choice. Nucleic Acid Res 22:4673-4680

Tietje C, Heinz E (1998) Uridine-diphospho-sulphoquinovose: diacylglycerol sulfoquinovosyl transferase activity is concentrated in the inner membrane of chloroplast envelope. Planta 206:72-78

Tranel PJ, Froehlich J, Goyal A, Keegstra K (1995) A component of the chloroplastic protein import apparatus is targeted to the outer envelope membrane via a novel pathway. EMBO J $14: 2436-2446$

Tranel PJ, Keegstra K (1996) A novel, bipartite transit peptide targets OEP75 to the outer membrane of the chloroplastic envelope. Plant Cell 8:2093-2104

Trnovsky J (1992) Semidry electroblotting of DNA and RNA from agarose and polyacrylamide gels. Biotechniques 13:800-802

Tsugeki R, Nishimura M (1993) Interaction of homologues of Hsp70 and Cpn60 with ferredoxin-NADP+ reductase upon its import into chloroplasts. FEBS Lett 320:198-202

VanderVere P, Benett R, Oblong JE, Lamppa GK (1995) A chloroplast processing enzyme involved in precursor maturation shares a zinc-binding motif with a recently recognized family of metalloendopeptidases. Proc Natl Acad Sci USA 92:7177-7181

Van't Hof R, van Klompenburg W, Pilon M, Kozubek A, de Korte-Kool G, Demel RA, Weisbeek PJ, de Kruijff B (1993) The transit sequence mediates the specific interaction of the precursor of ferredoxin with chloroplast envelope membrane lipids. $J$ Biol Chem 268:4037-4042

Vojta A, Alavi M, Becker T, Hörmann F, Küchler M, Soll J, Thomson R, Schleiff E (2004) The protein translocon of the plastid envelopes. J Biol Chem 279:21401-21405

Von Heijne G, Steppuhn J, Herrmann RG (1989) Domain structure of mitochondrial and chloroplast targeting peptides. Eur J Biochem 180:535-545

Waegemann K, Eichacker L and Soll J (1992) Outer envelope membranes from chloroplasts are isolated as right side-out vesicles. Planta 187:89-94

Waegemann K, Soll J (1991) Characterization of the protein import apparatus in isolated outer envelopes of chloroplats. Plant J 1:149-158

Waegemann K, Soll J (1995) Characterization and isolation of the chloroplast protein import machinery. Methods Cell Biol 50:255-267

Waegemann K, Soll J (1996) Phosphorilation of the transit sequence of chloroplast precursor proteins. J Biol Chem 271:6545-6554

Woodcock DM, Growther PJ, Doherty J, Jefferson S, DeCurz E, Noyer-Weidner M, Smith SS, Michael MZ, Graham MW (1989) Quantitative evolution of Escherichia coli host strains 
for tolerance of cytosine methylation in plasmid and phage recombinants. Nucl Acids Res $17: 3469-3478$

Wu C, Seibert FS, Ko K (1994) Identification of chloroplast envelope proteins in close physical proximity to a partially translocated chimeric precursor protein. J Biol Chem 269:32264-32271

Young ME, Keegstra K, Froehlich JE (1999) GTP promotes the formation of early-import intermediates but is not required during the translocation step of protein import into chloroplasts. Plant Physiol 121:237-244 


\section{Aknowledgements}

I thank Prof. Dr. Jürgen Soll for giving me the opportunity to perform this work, to develop new skills, collect new knowledge and to train the patience and persistence that are necessary for daily work in the laboratory.

I am grateful to Dr. Bettina Bölter for her time, useful suggestions and discussions that helped me a lot in my research and especially during the finalization of this thesis.

I am thankful to PD Dr. Enrico Schleiff for advises and discussions during the harder periods of my $\mathrm{PhD}$.

I specially thank Ahmed Nada, who devoted his precious time when I first came to the lab, who showed me nearly all techniques that I was using in this thesis, for all his help, support and friendship.

This way I want to thank Thomas for plenty of useful advises, protocols and discussions.

To my lab-colleagues Ewa, Rita and Monica I am unlimitly grateful for great working atmosphere, for fun in the lab, support in hard times and readiness to help. They managed to turn all my downs to ups by multitude of useful advices and by just being there.

I specially thank Marko, Lena, Thomas and Torsten for their friendship, understanding and creation of nice working atmosphere.

I thank Mislav, Fatima, Soumya and Johanna for nice daily small talks and for spreading the good mood.

Special thanks to Tihana for being a great friend and for making me feel very useful by avaking my teaching and demonstration skills.

And last, but not the least, I thank to my husband and colleague Aleksandar who encouraged me both scientifically and spiritually on the long journey during my whole $\mathrm{PhD}$ time, towards the finish of this thesis.

I can not thank enough to my family, especially my mother, who has always been there for me and enabled me to reach my goal, by supporting me throughout my education time and specially the last four years of my $\mathrm{PhD}$. 


\section{Curriculum vitae}

\section{Date and place of birth}

$11^{\text {th }}$ March 1979, Zagreb, Croatia

\section{Elementary school education}

1985-1993, Zagreb

\section{Secondary school education}

1993-1997, Gymnasium for Mathematics and Natural Sciences (V. Gymnasium), Zagreb

\section{Academic education}

1997-2002 Study of Molecular Biology at the Department of Biology, Faculty of Science, University of Zagreb

1999-2000 Research on the thesis "Chloroplast isomerase TLP40 gene homologue sll0408 has the important role in cyanobacterium Synechocystis PCC6803 biogenesis" awarded by The Rector award in 2000 (Performed at the Ruđer Bošković Institute, Zagreb, under the supervision of Prof. Dr. Nikola Ljubešić and Dr. Hrvoje Fulgosi)

2000-2002 Diploma degree research "Activity of retrotranspozon Tpv2 in Arabidopsis thaliana L." (Performed at the Department of Molecular Biology, Faculty of Science, University of Zagreb, under the supervision of Prof. Dr. Dražena Papeš and Dr. Srećko Jelenić). Graduated in October, 2002

2002- Since November 2002, PhD thesis in the lab of Prof. Dr. Jürgen Soll at the Botanical Institute, Faculty of Biology, Ludwig-Maximilian University, Munich 


\section{Ehrenwörtliche Versicherung}

Hiermit versichere ich dass die vorliegende Dissertation von mir selbständig und ohne unerlaubte Hilfe angefertigt wurde.

Lea Vojta

München, den 14. 11. 2006

\section{Erklärung}

Ich habe zuvor nicht versucht, anderweitig eine Dissertation einzureichen oder mich einer Doktorprüfung zu unterziehen. Die Dissertation wurde keiner weiteren Prüfungskommision weder in Teilen noch als Ganzes vorgelegt.

Lea Vojta

München, den 14. 11. 2006 